\title{
The e-ASTROGAM mission
}

\section{(exploring the extreme Universe with gamma rays in the $\mathrm{MeV}-\mathrm{GeV}$ range)}

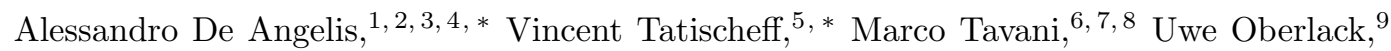
Isabelle A. Grenier, ${ }^{10}$ Lorraine Hanlon, ${ }^{11}$ Roland Walter, ${ }^{12}$ Andrea Argan, ${ }^{13}$ Peter von Ballmoos, ${ }^{14}$ Andrea Bulgarelli, ${ }^{15}$ Immacolata Donnarumma, ${ }^{6,16}$ Margarita Hernanz, ${ }^{17}$ Irfan Kuvvetli, ${ }^{18}$ Mark Pearce, ${ }^{19}$ Andrzej Zdziarski, ${ }^{20}$ Alessio Aboudan, ${ }^{21}$ Marco Ajello, ${ }^{22}$ Giovanni Ambrosi,${ }^{23}$ Denis Bernard,${ }^{24}$ Elisa Bernardini, ${ }^{25}$ Valter Bonvicini, ${ }^{26}$ Andrea Brogna, ${ }^{24}$ Marica Branchesi, ${ }^{27,}{ }^{28}$ Carl Budtz-Jørgensen, ${ }^{18}$ Andrei Bykov, ${ }^{29}$ Riccardo Campana, ${ }^{15}$ Martina Cardillo, ${ }^{6}$ Paolo Coppi, ${ }^{30}$ Domitilla De Martino, ${ }^{31}$

Roland Diehl, ${ }^{32}$ Michele Doro, ${ }^{1,33}$ Valentina Fioretti, ${ }^{15}$ Stefan Funk, ${ }^{34}$ Gabriele Ghisellini, ${ }^{35}$ J. Eric Grove, ${ }^{36}$ Clarisse Hamadache, ${ }^{37,}{ }^{38,39}$ Dieter H. Hartmann, ${ }^{22}$ Masaaki Hayashida, ${ }^{40}$ Jordi Isern, ${ }^{17}$ Gottfried Kanbach, ${ }^{41}$ Jürgen Kiener, ${ }^{37,38,39}$ Jürgen Knödlseder, ${ }^{42}$ Claudio Labanti, ${ }^{15}$ Philippe Laurent, ${ }^{43}$ Olivier Limousin, ${ }^{44}$ Francesco Longo, ${ }^{45,46}$ Karl Mannheim, ${ }^{47}$ Martino Marisaldi, ${ }^{48,}{ }^{15}$ Manel Martinez, ${ }^{49}$ Mario N. Mazziotta, ${ }^{50}$ Julie McEnery, ${ }^{51}$ Sandro Mereghetti, ${ }^{52}$ Gabriele Minervini, ${ }^{6}$ Alexander Moiseev ${ }^{53}$

Aldo Morselli, ${ }^{8}$ Kazuhiro Nakazawa, ${ }^{54}$ Piotr Orleanski, ${ }^{55}$ Josep M. Paredes,${ }^{56}$ Barbara Patricelli, ${ }^{57,58}$

Jean Peyré, ${ }^{37,38,39}$ Giovanni Piano, ${ }^{6}$ Martin Pohl, ${ }^{59}$ Harald Ramarijaona, ${ }^{37,38,39}$ Riccardo Rando, ${ }^{1,33}$ Ignasi Reichardt ${ }^{60}$ Marco Roncadelli, ${ }^{61,}{ }^{62}$ Rui Curado da Silva ${ }^{63}$ Fabrizio Tavecchio, ${ }^{35}$ David J. Thompson ${ }^{64}$ Roberto Turolla, ${ }^{65,66}$ Alexei Ulyanov, ${ }^{67}$ Andrea Vacchi, ${ }^{68}$ Xin Wu, ${ }^{69}$ and Andreas Zoglauer ${ }^{70}$

(On behalf of the e-ASTROGAM Collaboration)

${ }^{1}$ Istituto Nazionale di Fisica Nucleare, Sezione di Padova, I-35131 Padova, Italy

${ }^{2}$ Istituto Nazionale di Astrofisica, Padova, Italy

${ }^{3}$ Dipartimento di Matematica, Informatica e Fisica, Università di Udine, I-33100 Udine, Italy

${ }^{4}$ Laboratorio de Instrumentaçao e Particulas and Instituto Superior Tecnico, Lisboa, Portugal

${ }^{5}$ CSNSM, CNRS and University of Paris Sud, F-91405, Orsay, France

${ }^{6}$ INAF/IAPS, via del Fosso del Cavaliere 100, I-00133, Roma, Italy

${ }^{7}$ University of Roma "Tor Vergata", I-00133, Roma, Italy

${ }^{8}$ Istituto Nazionale di Fisica Nucleare, Sezione di Roma "Tor Vergata", I-00133 Roma, Italy

${ }^{9}$ Institute of Physics and PRISMA Excellence Cluster,

Johannes Gutenberg University Mainz, D-55099 Mainz, Germany

${ }^{10}$ AIM Paris-Saclay, CEA/IRFU, CNRS, Univ Paris Diderot, F-91191 Gif-sur-Yvette, France

${ }^{11}$ School of Physics, University College Dublin, Ireland

${ }^{12}$ University of Geneva, Chemin d'Ecogia 16, CH-1290 Versoix, Switzerland

${ }^{13}$ INAF Headquarters, Viale del Parco Mellini, 84, I-00136, Roma, Italy

${ }^{14}$ IRAP Toulouse, 9 av. du Colonel-Roche - BP 44 346, F-31028 Toulouse Cedex 4, France

${ }^{15}$ INAF/IASF Bologna, Via Gobetti 101, I-40129 Bologna, Italy

${ }^{16}$ Now at Agenzia Spaziale Italiana, Roma, Italy

${ }^{17}$ ICE (CSIC-IEEC), Campus UAB, Carrer Can Magrans $s / n$, E-08193 Cerdanyola del Valles, Barcelona, Spain

${ }^{18}$ DTU Space, National Space Institute, Technical University of Denmark, Kgs. Lyngby, Denmark

${ }^{19}$ KTH Royal Institute of Technology, Dept. of Physics, 10691 Stockholm, Sweden

${ }^{20}$ Nicolaus Copernicus Astronomical Center, Polish Academy of Sciences, Bartycka 18, PL-00-716 Warszawa, Poland

${ }^{21}$ Dept. of Physics and Astronomy University of Padova and INAF, via Marzolo 8, I-35131 Padova, Italy

${ }^{22}$ Department of Physics and Astronomy, Clemson University, Clemson, SC 29634, USA

${ }^{23}$ INFN Perugia, Perugia, Italy

${ }^{24}$ Institute of Physics and PRISMA Excellence Cluster,

Johannes Gutenberg University Mainz, 55099 Mainz, Germany

${ }^{25}$ DESY, Platanen Allee 6, D-15738 Zeuthen, Germany

${ }^{26}$ INFN Trieste, via A. Valerio, I-34127 Trieste, Italy

${ }^{27}$ Università degli Studi di Urbino, DiSPeA, I-61029 Urbino, Italy

${ }^{28}$ Istituto Nazionale di Fisica Nucleare, Sezione di Firenze, Italy

${ }^{29}$ Ioffe Institute, St.Petersburg 194021, Russia

${ }^{30}$ Department of Astronomy, Yale University, P.O. Box 208101, New Haven, CT 06520-8101, USA

${ }^{31}$ NAF - Osservatorio Astronomico di Capodimonte, Salita Moiariello 16, I-80131 Napoli, Italy

${ }^{32}$ Max Planck Institut fuer extraterrestrische Physik, Giessenbachstr.1,

D-85748 Garching, Germany; Excellence Cluster Universe, Germany

${ }^{33}$ Dipartimento di Fisica e Astronomia "G. Galilei", Università di Padova, I-35131 Padova, Italy

${ }^{34}$ Erlangen Centre for Astroparticle Physics, D-91058 Erlangen, Germany

${ }^{35}$ INAF - Osservatorio di Brera, via E. Bianchi 46, I-23807 Merate, Italy

${ }^{36}$ U.S. Naval Research Laboratory, 4555 Overlook Ave SW, Washington, DC 20375, USA

${ }^{37}$ CSNSM, F-91405 Orsay Campus, France 


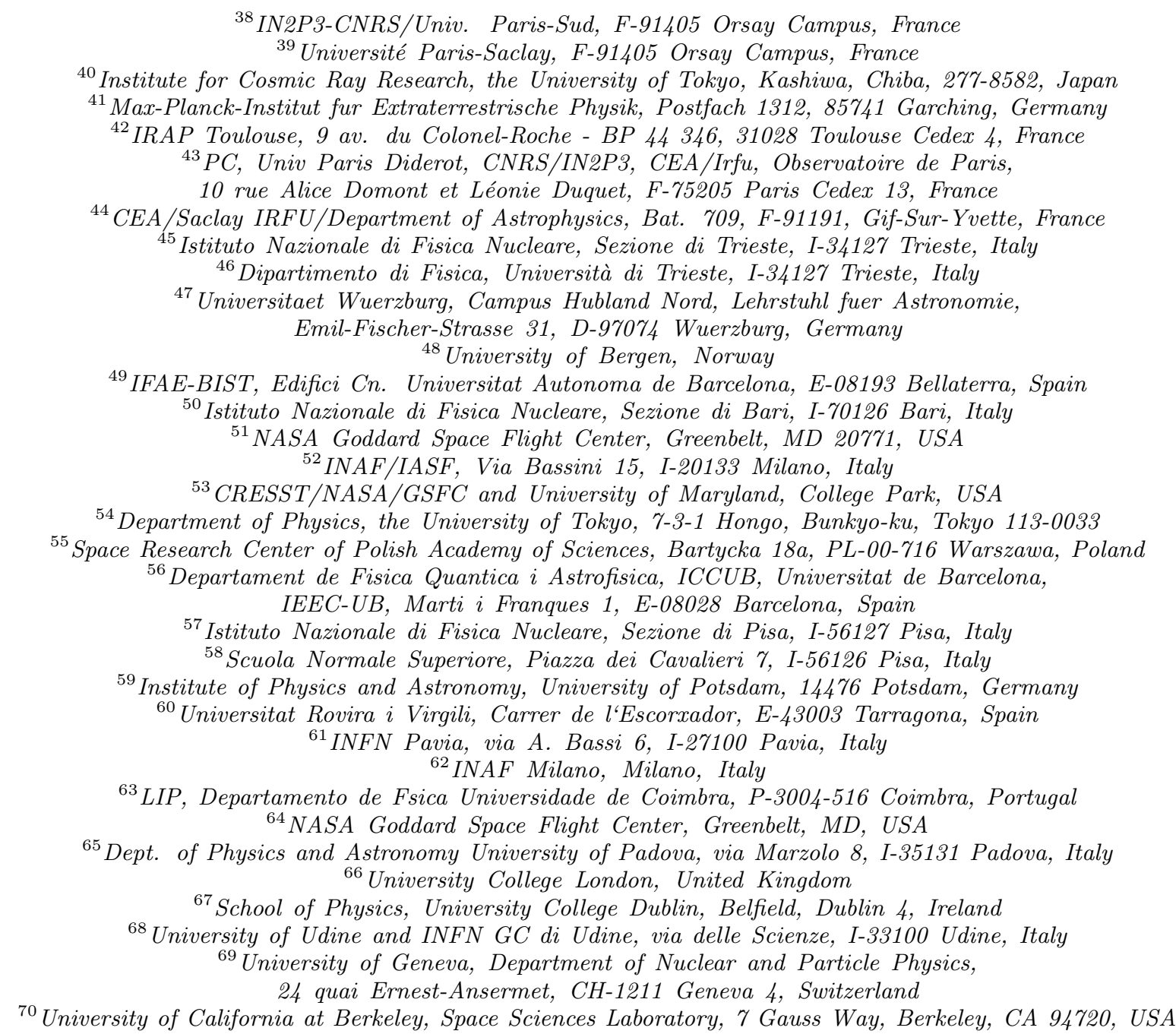

e-ASTROGAM ('enhanced ASTROGAM') is a breakthrough Observatory space mission, with a detector composed by a Silicon tracker, a calorimeter, and an anticoincidence system, dedicated to the study of the non-thermal Universe in the photon energy range from $0.3 \mathrm{MeV}$ to $3 \mathrm{GeV}-$ the lower energy limit can be pushed to energies as low as $150 \mathrm{keV}$, albeit with rapidly degrading angular resolution, for the tracker, and to $30 \mathrm{keV}$ for calorimetric detection. The mission is based on an advanced space-proven detector technology, with unprecedented sensitivity, angular and energy resolution, combined with polarimetric capability. Thanks to its performance in the MeV-GeV domain, substantially improving its predecessors, e-ASTROGAM will open a new window on the non-thermal Universe, making pioneering observations of the most powerful Galactic and extragalactic sources, elucidating the nature of their relativistic outflows and their effects on the surroundings. With a line sensitivity in the MeV energy range one to two orders of magnitude better than previous generation instruments, e-ASTROGAM will determine the origin of key isotopes fundamental for the understanding of supernova explosion and the chemical evolution of our Galaxy. The mission will provide unique data of significant interest to a broad astronomical community, complementary to powerful observatories such as LIGO-Virgo-GEO600-KAGRA, SKA, ALMA, E-ELT, TMT, LSST, JWST, Athena, CTA, IceCube, KM3NeT, and the promise of eLISA.

Keywords: High-Energy Gamma-Ray Astronomy, High-Energy Astrophysics, Nuclear Astrophysics, Compton and Pair Creation Telescope, Gamma-Ray Bursts, Active Galactic Nuclei, Jets, Outflows, Multiwavelength Observations of the Universe, Counterparts of gravitational waves, Fermi, Dark Matter, Nucleosynthesis, Early Universe, Supernovae, Cosmic Rays, Cosmic Antimatter

PACS numbers: PACS 95.55 Ka, PACS 98.70 Rz, 26.30.-k

* Corresponding Author 


\section{CONTENTS}

I. Introduction

II. Science Case

A. Processes at the heart of the extreme Universe: prospects for the Astronomy of the 2030s

1. Gamma-Ray Bursts

2. e-ASTROGAM and the new Astronomy

B. The origin and impact of high-energy particles on Galaxy evolution, from cosmic rays to antimatter

1. What are the CR energy distributions produced inside SNRs and injected into the surrounding ISM?

2. How do CR fluxes vary with Galactic environments, from passive interstellar clouds to active starburst regions and near the Galactic Center?

3. Where are the low-energy CRs and how do they penetrate dense clouds?

4. The origin and energy content of Galactic wind and Fermi bubbles

5. Antimatter and WIMP Dark Matter

C. Nucleosynthesis and the chemical evolution of our Galaxy

1. What are the progenitor system(s) and explosion mechanism(s) of thermonuclear SNe? Can we use SN Ia for precision cosmology?

2. How do core-collapse supernovae (CCSNe) explode? What is the recent history of CCSNe in the Milky Way?

3. Nova explosions

4. How are cosmic isotopes created in stars and distributed in the interstellar medium?

D. Observatory science in the $\mathrm{MeV}-\mathrm{GeV}$ domain

III. Scientific Requirements

IV. The Scientific Instrument

A. Measurement principle and payload overview

1. Silicon Tracker

2. Calorimeter

3. Anticoincidence System

4. Data Handling and Power Supply

5. Trigger logic and data flow architecture

B. Performance assessment

1. Background model

2. Angular and spectral resolution

3. Field of View

4. Effective area and continuum sensitivity

5. Line sensitivity

6. Polarization response
C. Technology readiness

31

V. Mission Configuration and Profile

31

A. Orbit and launcher

B. Spacecraft and system requirements

31

1. Attitude and Orbital Control Systems

2. Thermal control system

32

33

VI. Summary

33

Acknowledgments

33

References

33

\section{INTRODUCTION}

e-ASTROGAM is a gamma-ray mission concept proposed as a response to the European Space Agency (ESA) Call for the fifth Medium-size mission (M5) of the Cosmic Vision Science Programme. The planned launch date is 2029 .

The main constituents of the e-ASTROGAM payload will be:

- A Tracker in which the cosmic $\gamma$-rays can undergo a Compton scattering or a pair conversion, based on 56 planes of double-sided Si strip detectors, each plane with total area of $\sim 1 \mathrm{~m}^{2}$;

- A Calorimeter to measure the energy of the secondary particles, made of an array of CsI (Tl) bars of $5 \times 5 \times 80 \mathrm{~mm}^{3}$ each, with relative energy resolution of $4.5 \%$ at $662 \mathrm{keV}$;

- An Anticoincidence system (AC), composed of a standard plastic scintillator AC shielding and a Time of Flight, to veto the charged particle background.

If selected, e-ASTROGAM will operate in a maturing gravitational wave and multimessenger epoch, opening up entirely new and exciting synergies. The mission will provide unique and complementary data of significant interest to a broad astronomical community, in a decade of powerful observatories such as LIGO-Virgo-GEO600KAGRA, SKA, ALMA, E-ELT, LSST, JWST, Athena, CTA and the promise of eLISA.

The core mission science of e-ASTROGAM addresses three major topics of modern astrophysics.

- Processes at the heart of the extreme Universe: prospects for the Astronomy of the $2030 s$

Observations of relativistic jet and outflow sources (both in our Galaxy and in active galactic nuclei, AGNs) in the X-ray and $\mathrm{GeV}-\mathrm{TeV}$ energy ranges have shown that the $\mathrm{MeV}-\mathrm{GeV}$ band holds the key to understanding the transition from the low energy continuum to a spectral range shaped by 
very poorly understood particle acceleration processes. e-ASTROGAM will: (1) determine the composition (hadronic or leptonic) of the outflows and jets, which strongly influences the environment - breakthrough polarimetric capability and spectroscopy providing the keys to unlocking this longstanding question; (2) identify the physical acceleration processes in these outflows and jets (e.g. diffusive shocks, magnetic field reconnection, plasma effects), that may lead to dramatically different particle energy distributions; (3) clarify the role of the magnetic field in powering ultrarelativistic jets in gamma-ray bursts (GRBs), through timeresolved polarimetry and spectroscopy. In addition, measurements in the e-ASTROGAM energy band will have a big impact on multimessenger astronomy in the 2030s. Joint detection of gravitational waves and gamma-ray transients would be groundbreaking.

- The origin and impact of high-energy particles on galaxy evolution, from cosmic rays to antimatter

e-ASTROGAM will resolve the outstanding issue of the origin and propagation of low-energy cosmic rays affecting star formation. It will measure cosmic-ray diffusion in interstellar clouds and their impact on gas dynamics and state; it will provide crucial diagnostics about the wind outflows and their feedback on the Galactic environment (e.g., Fermi bubbles, Cygnus cocoon). e-ASTROGAM will have optimal sensitivity and energy resolution to detect line emissions from $511 \mathrm{keV}$ up to $10 \mathrm{MeV}$, and a variety of issues will be resolved, in particular: (1) origin of the gamma-ray and positron excesses toward the Galactic inner regions; (2) determination of the astrophysical sources of the local positron population from a very sensitive observation of pulsars and supernova remnants (SNRs). As a consequence e-ASTROGAM will be able to discriminate the backgrounds to dark matter (DM) signals.

- Nucleosynthesis and the chemical enrichment of our Galaxy

The e-ASTROGAM line sensitivity is more than an order of magnitude better than previous instruments. The deep exposure of the Galactic plane region will determine how different isotopes are created in stars and distributed in the interstellar medium; it will also unveil the recent history of supernova explosions in the Milky Way. Furthermore, e-ASTROGAM will detect a significant number of Galactic novae and supernovae in nearby galaxies, thus addressing fundamental issues in the explosion mechanisms of both core-collapse and thermonuclear supernovae. The $\gamma$-ray data will provide a much better understanding of Type Ia supernovae and their evolution with look-back time and metal- licity, which is a pre-requisite for their use as standard candles for precision cosmology.

In addition to addressing its core scientific goals, eASTROGAM will achieve many serendipitous discoveries (the unknown unknowns) through its combination of wide field of view (FoV) and improved sensitivity, measuring in 3 years the spectral energy distributions of thousands of Galactic and extragalactic sources, and providing new information on solar flares and terrestrial gamma-ray flashes (TGF). e-ASTROGAM will become a key contributor to multiwavelength time-domain astronomy. The mission has outstanding discovery potential as an Observatory facility that is open to a wide astronomical community.

e-ASTROGAM is designed to achieve:

- Broad energy coverage (0.3 MeV to $3 \mathrm{GeV})$, with one-two orders of magnitude improvement in continuum sensitivity in the range $0.3 \mathrm{MeV}-100 \mathrm{MeV}$ compared to previous instruments (the lower energy limit can be pushed to energies as low as 150 $\mathrm{keV}$, albeit with rapidly degrading angular resolution, for the tracker, and to $30 \mathrm{keV}$ for calorimetric detection);

- Unprecedented performance for $\gamma$-ray lines, with, for example, a sensitivity for the $847 \mathrm{keV}$ line from Type Ia SNe 70 times better than that of INTEGRAL/SPI;

- Large FoV (>2.5 sr), ideal to detect transient sources and hundreds of GRBs;

- Pioneering polarimetric capability for both steady and transient sources;

- Optimized source identification capability afforded by the best angular resolution achievable by stateof-the-art detectors in this energy range (about 0.15 degrees at $1 \mathrm{GeV}$ );

- Sub-millisecond trigger and alert capability for GRBs and other cosmic and terrestrial transients;

- Combination of Compton and pair-production detection techniques allowing model-independent control on the detector systematic uncertainties.

\section{SCIENCE CASE}

e-ASTROGAM will open the $\mathrm{MeV}$ region for exploration, with an improvement of one-two orders of magnitude in sensitivity (Fig. 1) compared to the current state of the art, much of which was derived from the COMPTEL instrument more than two decades ago. It will also achieve a spectacular improvement in terms of source localization accuracy (Fig. 2) and energy resolution, and will allow to measure the contribution to the radiation of the Universe in an unknown range (Fig. 3). The sensitivity of e-ASTROGAM will reveal the transition from 


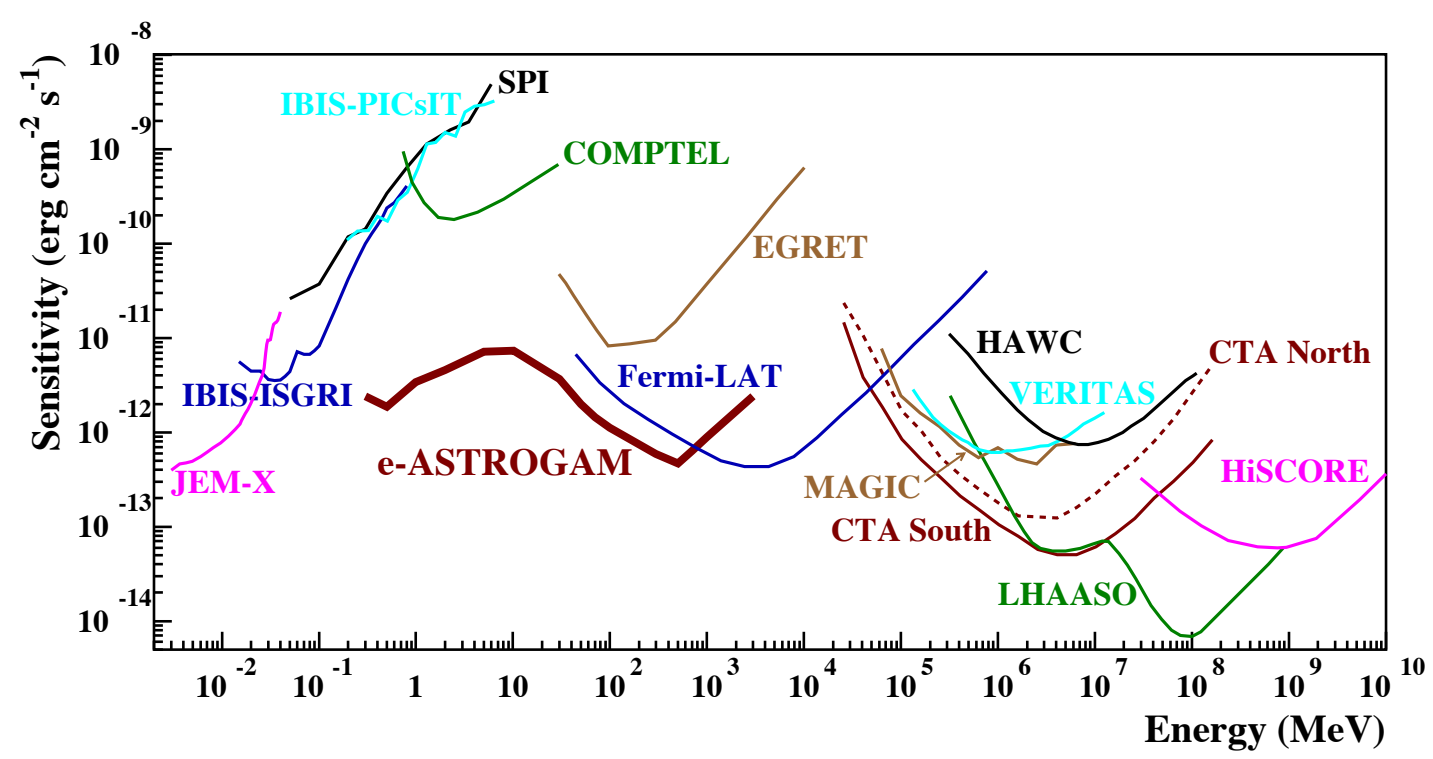

FIG. 1. Point source continuum differential sensitivity of different X- and $\gamma$-ray instruments. The curves for INTEGRAL/JEMX, IBIS (ISGRI and PICsIT), and SPI are for an effective observation time $T_{\mathrm{obs}}=1 \mathrm{Ms}$. The COMPTEL and EGRET sensitivities are given for the typical observation time accumulated during the $\sim 9$ years of the CGRO mission (see Fig. 1 in [129]). The Fermi/LAT sensitivity is for a high Galactic latitude source in 10 years of observation in survey mode. For MAGIC, VERITAS (sensitivity of H.E.S.S. is similar), and CTA, the sensitivities are given for $T_{\mathrm{obs}}=50$ hours. For HAWC $T_{\mathrm{obs}}=5$ yr, for LHAASO $T_{\mathrm{obs}}=1 \mathrm{yr}$, and for HiSCORE $T_{\mathrm{obs}}=1000 \mathrm{~h}$. The e-ASTROGAM sensitivity is calculated at $3 \sigma$ for an effective exposure of 1 year and for a source at high Galactic latitude.

nuclear processes to those involving electro- and hydrodynamical, magnetic and gravitational interactions.

An important characteristic of e-ASTROGAM is its ability to measure polarization in the $\mathrm{MeV}$ range, which is afforded by Compton interactions in the detector. Polarization encodes information about the geometry of magnetic fields and adds a new observational pillar, in addition to the temporal and spectral, through which fundamental processes governing the $\mathrm{MeV}$ emission can be determined. The addition of polarimetric information will be crucial for a variety of investigations, including accreting black-hole (BH) systems, magnetic field structures in jets, and the emission mechanisms of GRBs. Polarization will provide definitive insight into the presence of hadrons in extragalactic jets and the origin of ultrahigh-energy cosmic rays (CR).

In the following sections, the core science questions 50 to be addressed by e-ASTROGAM are presented. The requirements coming from the scientific objectives, and driving the instrument design, are presented in Sect. III.

\section{A. Processes at the heart of the extreme Universe: prospects for the Astronomy of the 2030s}

The Universe accessible to e-ASTROGAM is dominated by strong particle acceleration. Ejection of plasma (jets or uncollimated outflows), ubiquitous in accreting systems, drives the transition from the keV energy range, typical of the accretion regime, to the $\mathrm{GeV}-\mathrm{TeV}$ range, through reprocessing of synchrotron radiation (e.g, inverse Compton, IC) or hadronic mechanisms. For some sources the $\mathrm{MeV}$ band naturally separates the acceleration and reprocessing energy ranges. Other systems, instead, radiate the bulk of their output in the $\mathrm{MeV}$ band. This is the most frequent case for AGNs at cosmological distances.

e-ASTROGAM will also study extreme acceleration mechanisms from compact objects such as neutron stars and (supermassive) black holes. Its polarimetric capabilities and its continuum sensitivity will solve the problem of the nature of the highest energy radiation.

The transition to non-thermal processes involves, in particular, the emission of relativistic jets and winds. In our Galaxy, this is relevant for compact binaries and microquasars. The interplay between accretion processes and jet emission can best be studied in the $\mathrm{MeV}$ region, where disk Comptonization is expected to fade and other non-thermal components can originate from jet particles. e-ASTROGAM observations of Galactic compact objects and in particular of accreting $\mathrm{BH}$ systems (such as Cygnus X-1 [151, Cygnus X-3 (3, 133), V404 Cygni [119]) will determine the nature of the steady-state emission due to Comptonization and the transitions to highly non-thermal radiation (Fig. 4). The main processes behind this emission are Compton scattering by accelerated non-thermal electrons and its attenuation/reprocessing by electron-positron pair production. The magnetic field in the BH vicinity can be quite strong, and have both random and ordered components; synchrotron emission 

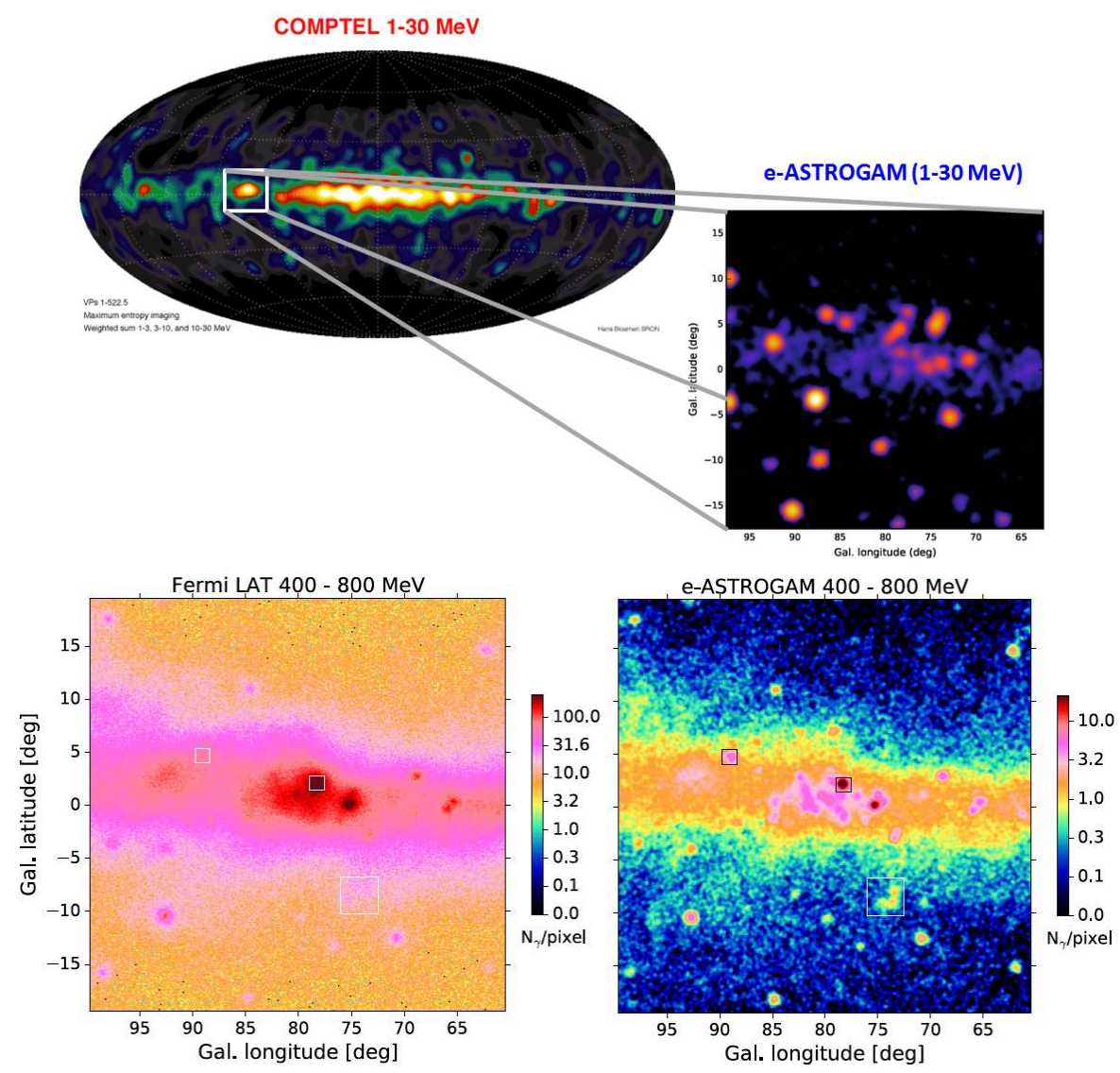

FIG. 2. An example of the capability of e-ASTROGAM to transform our knowledge of the MeV-GeV sky. Upper panel: The upper left figure shows the 1-30 MeV sky as observed by COMPTEL in the 1990s; the lower right figure shows the simulated Cygnus region in the 1-30 MeV energy region from e-ASTROGAM. Lower panel: comparison between the view of the Cygnus region by Fermi in 8 years (left) and that by e-ASTROGAM in one year of effective exposure (right) between $400 \mathrm{MeV}$ and $800 \mathrm{MeV}$.

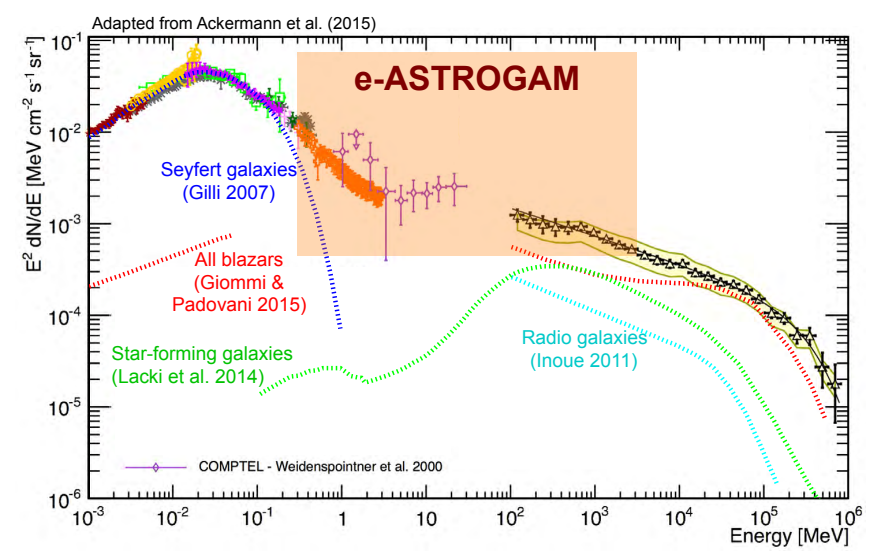

FIG. 3. Compilation of the measurements of the total extragalactic gamma-ray intensity between $1 \mathrm{keV}$ and $820 \mathrm{GeV}$ [13], with different components from current models; the contribution from MeV blazars is largely unknown. The semitransparent band indicates the energy region in which eASTROGAM will dramatically improve on present knowledge. by the electrons in the accretion flow may give rise to polarized $\mathrm{MeV}$ emission (e.g. [117]), which can be measured by e-ASTROGAM, together with spectral transitions. Signatures of $e^{+} e^{-}$production and annihilation (e.g. [125]) can be detected by e-ASTROGAM.

e-ASTROGAM offers a unique way to study accelerated jets in blazars on short and long timescales. Among the unsolved questions are the origin of the photons undergoing Comptonization, the location of the acceleration region in jets (near to, or far from, the central black hole), and the presence of additional components of accelerated electrons i.e. whether or not there is a mildly relativistic population of electrons. The latter question has important implications for the understanding of acceleration processes such as shock or magnetic field reconnection.

Very fast variations of the gamma-ray emission have been recently detected and are challenging current models; an example is provided by the flat spectrum radio quasar (FSRQ) 3C 279 [15]. During a flare in 2015 [15], variations of 2-3 minutes were detected in gamma-rays, i.e. well below standard light-travel times usually assumed in theoretical models. These very rapid variability phenomena are the most compelling evidence of the oc- 

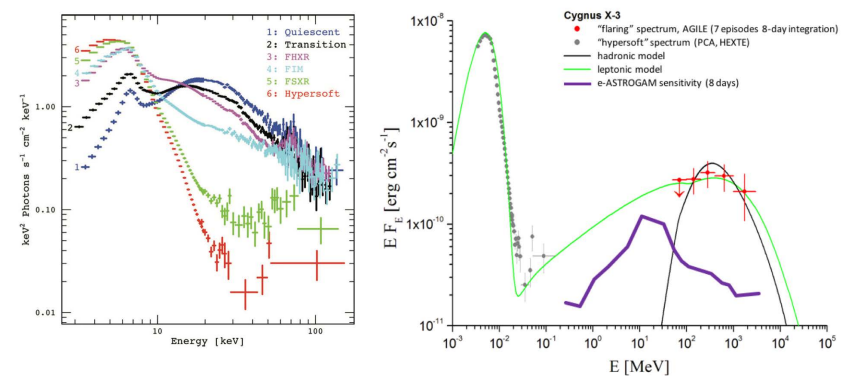

FIG. 4. Left panel: Different states of Cyg X-3 in the soft $\mathrm{X}$-ray/hard X-ray ranges (adapted from [85]). Right panel: spectral energy distribution (SED) of Cyg X-3 during the $\gamma-$ ray flaring activity in 2011 (adapted from [11]). The purple curve is the e-ASTROGAM $3 \sigma$ sensitivity for an 8-day observation, from a simulation taking into account the expected background; the time matches the integration time of the AGILE $\gamma$-ray spectrum (red points). The green curve refers to a leptonic model and the black curve to a hadronic model.

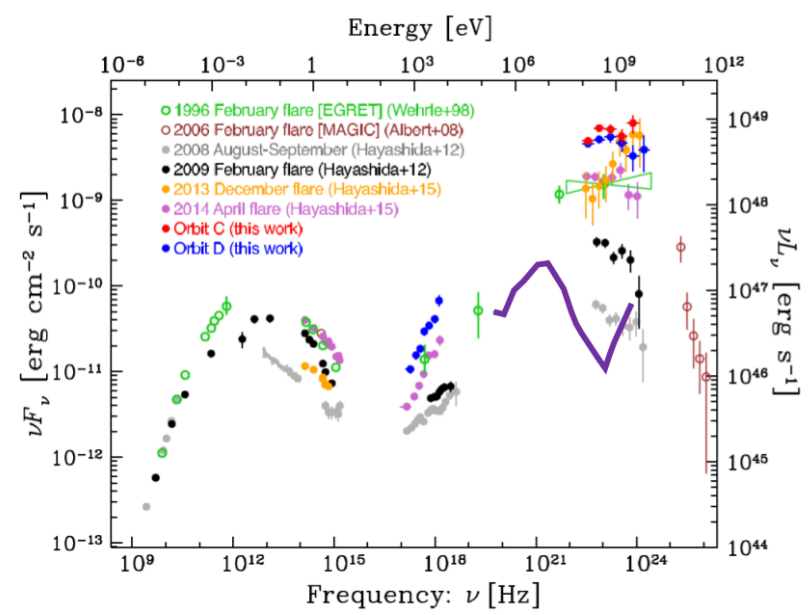

FIG. 5. SED from a collection of different spectral states of the FSRQ 3C 279 showing a dramatic gamma-ray flaring activity, including the minute-timescale episode detected by Fermi in June 2015 [15]. The purple solid line is the $3 \sigma$ eASTROGAM sensitivity calculated for a $50 \mathrm{ks}$ exposure.

currence of out-of-equilibrium particle acceleration most likely produced by magnetic field reconnection with remarkably high efficiency. On a smaller scale the magnetic field merging and the acceleration of relativistic electrons and ions have been typically observed in solar flares, where sub-second timescales and gamma-ray emissions into the $100 \mathrm{MeV}$ range have been frequently detected. This process, called "super-acceleration" (see [136]), has recently been invoked to explain the unexpected gammaray flare of the Crab Nebula ([5, 135]), and leads to a very efficient mechanism for magnetic energy dissipation. It may apply to very rapid gamma-ray emission from compact objects with timescales and intensities incompatible with the current paradigms. e-ASTROGAM will provide crucial information in the strongly variable spectral range
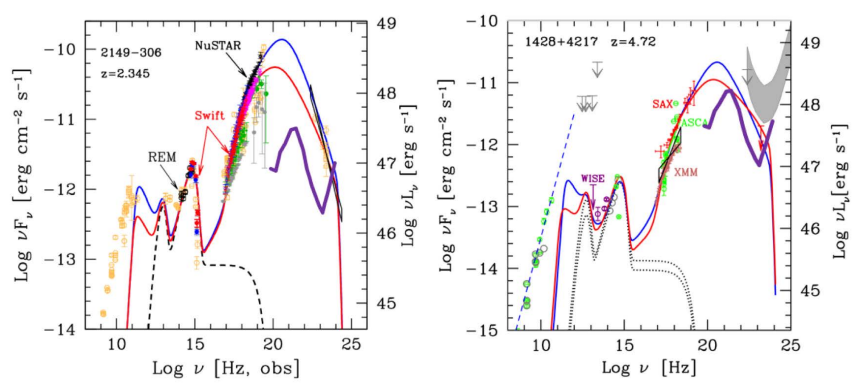

FIG. 6. SED of PKS 2149-306 at $z=2.345$ (left panel) and the fitting model by [128] and GB $1428+4217$ at $z=4.72$ (right panel) and the fitting model by Ghisellini (private communication). Downward arrow is $2 \sigma$ Fermi upper limit over 7.5 years (see [106]). The sensitivity of e-ASTROGAM, calculated for an effective exposure of 1 year, is shown as a purple curve in the $0.3 \mathrm{MeV}-3 \mathrm{GeV}$ range. The bulk of the power is expected in the $\mathrm{MeV}$ band, around $10^{21} \mathrm{~Hz}$.

1-100 MeV (Fig. 5), constraining the electron population spectrum and the responsible particle acceleration mechanisms. An outstanding unsolved issue is the existence of gamma-rays produced by hadronic processes. The origin of photons can be effectively probed both by much improved spectral measurements in the MeV-GeV band (detecting the "pion bump"), and by polarimetric observations. Polarimetry is indeed a powerful tool to establish the nature of the emitters (hadrons vs. leptons) and, in case of leptonic (i.e., inverse Compton) emission, the nature of the soft photon target radiation [152].

An example is the very bright (about 50 times our sensitivity at $1 \mathrm{MeV}$ ) AGN 3C279 [152. In the $100 \mathrm{keV}-10$ $\mathrm{MeV}$ range, the polarization in leptonic emission models is low and rapidly decreasing with energy (from $40 \%$ to 0 ), while for hadronic models it is high and increasing in energy $(>60 \%$ to $80 \%)$. Even in non optimal conditions (fields not well ordered, alignment to line of sight not optimal) the polarization signature would allow to identify unambiguously a hadronic scenario. The best targets for this study are blazars - both BL Lacs (suitable to study the transition from the synchrotron to the IC or hadronic-dominated component) and the powerful FSRQs (in which the transition from the highly polarized SSC radiation to the less polarized external Compton component can be revealed).

Over $2 / 3$ of the 3033 sources from the 3rd Fermi-LAT Catalog of $\mathrm{GeV}$-band sources (3FGL), have power-law spectra (at energies larger than $100 \mathrm{MeV}$ ) steeper than $E^{-2}$, implying that their peak energy output is below 100 $\mathrm{MeV}$ (Fig. 6). The subset that is extragalactic hosts BHs with masses reaching $10^{10} M_{\odot}$, and are often located at high redshift $(z \geq 2-3)$. They are therefore ideal tracers of the formation and history of super-massive BHs in the Universe [65, 66]. In particular, the sources hosting the most massive $\mathrm{BH}$ are elusive in the $\mathrm{GeV}$ band as probed by Fermi-LAT.

Recent hard X-ray surveys 19, 20, 65 have been shown to be more effective in detecting higher redshift 
blazars compared to $\mathrm{GeV} \gamma$-ray surveys. The main reason is that the SEDs of these sources peak in the $\mathrm{MeV}$ region (hereafter, MeV blazars) and detection becomes a difficult task for $\gamma$-ray instruments, even for Fermi-LAT. eASTROGAM will detect hundreds of these blazars, constraining their SED peaks very tightly (Fig. 6). These discoveries will revolutionize our understanding of: (i) how the two populations of AGNs (radio-quiet and radioloud) evolve with redshift; (ii) the formation and growth of supermassive BHs; (iii) the connection between the jet and the central engine and (iv) the role of the jet in the feedback occurring in the host galaxies [142]. eASTROGAM will very substantially advance our knowledge of MeV-blazars up to redshift $\sim 4.5$, with implications for blazar physics and cosmology - the attenuation due to cosmological absorption is negligible in the $\mathrm{MeV}$ region and the sensitivity has been shown by fits to the SED to be adequate (Fig. 6). These observations will be invaluable and complementary to data from the future ATHENA mission for the study of super-massive black holes.

By detecting the population of $\mathrm{MeV}$-blazars up to redshift $\sim 4.5$, e-ASTROGAM will resolve the extragalactic gamma-ray background (EGB) in the $\mathrm{MeV}$ range (Fig. 3). A possible residual excess in the $\mathrm{MeV}$ range may have cosmological implications related to baryogenesis [143. Quasar-driven outflows could also account for the yetnot-understood part of the EGB [146].

\section{Gamma-Ray Bursts}

GRBs are among the most intriguing and puzzling phenomena in astrophysics. They are believed to originate from coalescing NSs or BHs (short-GRBs, of duration $\lesssim 2 \mathrm{~s}$ ) or from the final collapses of massive stars (longGRBs). Their radiative output is believed to originate from highly relativistic outflows.

e-ASTROGAM will allow unprecedented studies of both classes of GRBs, thanks to the combined interplay between the imaging Tracker, the Calorimeter, and the AC system, providing excellent sensitivity for spectral and timing studies combined with polarization capability. It will be possible to detect GRBs with durations from sub-millisecond to hundreds of seconds and study them in the energy range where their emission peaks. The e-ASTROGAM spectral performance will be very relevant, because of the role played by the $\mathrm{MeV}$ range in constraining theoretical models of particle acceleration. The total number of GRBs detectable by e-ASTROGAM is estimated to be $\sim 600$ during the first 3 years (see Sect. IV B 6).

The e-ASTROGAM imaging Tracker can localize GRBs within $0.1^{\circ}-1^{\circ}$ (depending on their intensity), and the information can be processed onboard for a fast communication. The delay of the alerts is similar to FermiLAT, being the procedure (event rates onboard, plus simple localization based on onboard fast reconstruction) the same. This translates into an initial alert with an accuracy of $1^{\circ}-2^{\circ}$ within $(2-4) \mathrm{s}$, to be confirmed with similar accuracy within $30 \mathrm{~s}$ and then made public. Within $4 \mathrm{~h}-8 \mathrm{~h}$ the final alert accuracy of $0.1^{\circ}-1^{\circ}$ can be reached. The alerts issued by e-ASTROGAM will be extremely valuable for observatories such as CTA. The Calorimeter can act as an independent detector extending the energy range down to $30 \mathrm{keV}$ : an on-board trigger logic spanning timescales from sub-ms up to seconds will be implemented.

For bright GRBs, e-ASTROGAM will detect polarization in the $\mathrm{MeV}$ range. The Tracker can provide information down to (150-200) keV, also for polarization measurements. We can estimate as $42 \mathrm{GRBs} /$ year the number of events with a detectable polarization fraction of $20 \%$; for a polarization fraction of $10 \%$ the number is about $16 \mathrm{GRBs} /$ year. The polarization information, combined with spectroscopy in the $\mathrm{MeV}-\mathrm{GeV}$ band, will provide a unique diagnostic to address the role of magnetic fields in the radiative output and dynamics of the most relativistic outflows in our Universe.

The e-ASTROGAM sensitivity to short and long GRBs will be very useful also for the detection of electromagnetic counterparts of impulsive gravitational wave events, as described in Sect. II A2

\section{2. e-ASTROGAM and the new Astronomy}

e-ASTROGAM fills the need for an MeV gamma-ray detector operating at the same time as facilities such as SKA and CTA, as well as eLISA and neutrino detectors. It guarantees the availability of complementary information to obtain a coherent picture of the transient sky and the sources of gravitational waves (GWs) and highenergy neutrinos. This will undoubtedly be an exciting new landscape for astronomy in the XXI century.

The first detections of GW signals from binary black hole (BH-BH) mergers, observed by Advanced LIGO 2, marked the onset of the era of GW astronomy. The next breakthrough will be the observation of their electromagnetic counterparts, which will characterize the progenitor and its environment. Neutron star (NS)-BH or NSNS mergers can eject relativistic outflows 98, 141, 145. or produce sub-relativistic omnidirectional high-energy emission [130]. In both cases emission up to the $\mathrm{MeV}$ energy range can be expected. The expected detection rate of GRB prompt emission by e-ASTROGAM in coincidence with a GW detection is up to 1.5 events per year [107]; it will double after the incorporation of KAGRA and LIGO-India into the GW network, which should happen several years before 2029. e-ASTROGAM will also play a key role in the multiwavelength study of GW events: in fact, its large FoV will maximize the detection probability and provide accurate sky localization $(<1$ sq. deg at $1 \mathrm{MeV}$ ), thus allowing the follow-up of the GW events by other telescopes. This capability will be crucial for the identification and the multiwavelength char- 
acterization of the GW progenitor and of its host galaxy. e-ASTROGAM could associate binary systems to short GRBs, improving the localization of sources and measuring spectral energy distributions.

e-ASTROGAM may coincide with the third generation of ground-based interferometer projects, such as the Einstein Telescope [113, and Cosmic Explorer 92, with an order of magnitude increase in sensitivity. Furthermore, the space detector eLISA [51] will open GW observations to massive $\left(10^{4}-10^{6}\right) M_{\odot} \mathrm{BHs}$, which could have magnetized circumbinary disks powering EM emission. Simultaneous GW/EM emission will transform our understanding of the formation, evolution, properties and environment of different mass compact objects through cosmic history.

Another important topic in the new astronomy will be neutrino astrophysics. Although astrophysical neutrinos have been detected by IceCube [1, no significant cluster (in space or in time) has been found yet. Coincidences in time and space of astrophysical neutrino events with exceptional flares of blazars have been proposed by several authors (e.g. 80]). Among the speculated possible origins of the IceCube events are GRBs with jets shocked in surrounding matter. Models explaining hypernovae and low luminosity GRBs (e.g. [124]) predict neutrino and gamma-ray emission, with the highest energy $\gamma$-rays being likely absorbed, and thus a possible cutoff in the $\mathrm{MeV}$ range.

Observations by e-ASTROGAM could therefore open a new avenue within multimessenger astrophysics, also by allowing multimessenger coincidences for KM3NeT in the Mediterranean sea, and thus making it possible to remove the background for neutrinos in the $\mathrm{TeV}$ range.

B. The origin and impact of high-energy particles
on Galaxy evolution, from cosmic rays to antimatter

Relativistic particles permeate the interstellar medium (ISM) of galaxies and drive their evolution by providing heat, pressure and ionization to the clouds and to galactic winds and outflows. Sub-GeV particles have a particularly important role and understanding their origin and transport has profound implications. High-energy particles also signal the presence of antimatter and potential sources of dark matter. Observations with eASTROGAM can advance our knowledge on all fronts by observing the radiation borne from particle interactions with interstellar gas: electrons emitting bremsstrahlung $\gamma$ rays (often dominant below $50-100 \mathrm{MeV}$ ); nuclei producing $\pi^{0}$ s decaying into $\gamma$ rays (with the characteristic "pion bump" in energy density below one $\mathrm{GeV}$ ); nuclear excitation lines, and the $511-\mathrm{keV}$ line from positron annihilation. In addition to the the gas-related emission, e-ASTROGAM will also observe the large-scale emission due to IC scattering of CR electrons on the interstellar radiation field and cosmic microwave background. This is a significant component above $\sim 100 \mathrm{MeV}$, and it is be- lieved to be the dominant interstellar diffuse component below few tens of $\mathrm{MeV}$.

We have fair measurements of the local spectrum of Galactic cosmic-rays (CRs), from $\mathrm{GeV}$ to $\mathrm{PeV}$ energies, but not at lower energies. There is only a very coarse description of their flux both radially and vertically across the Milky Way. Their diffusion processes in and out of the spiral arms and star-forming regions are poorly understood, as is their penetration through dense clouds as a function of energy. Convincing, albeit not definitive, observational evidence is available for $\mathrm{CR}$ acceleration by supernova shockwaves (via diffusive shock acceleration), but little understanding of the total energy imparted to CRs, of their escape into the ambient medium, of their diffusion through stellar-wind-driven turbulence in starburst regions, and of their role in the self-regulation of the Galactic ecosystem.

\section{What are the CR energy distributions produced inside SNRs and injected into the surrounding ISM?}

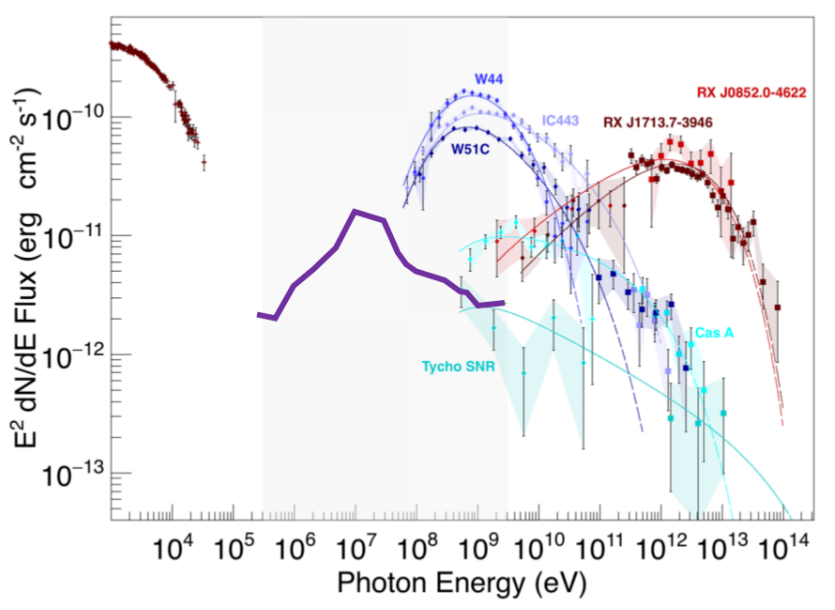

FIG. 7. e-ASTROGAM sensitivity for 1-year exposure (thick purple line) compared to typical $\gamma$-ray energy spectra for several SNRs; young SNRs ( $<1000$ years) are shown in green. High-energy data $(E>100 \mathrm{MeV})$ are taken from [60; lowenergy data (related to RX J1713.7-3946) from [131.

Fermi-LAT has barely resolved a handful of SNRs, indicating that younger ones tend to emit harder gammarays [6], and detected curved spectra compatible with pion bumps in only three sources (IC 443, W44, W51C, [9, 78]). The performance of e-ASTROGAM will open the way for spectral imaging of a score of SNRs, spanning ages from $10^{3}$ to $10^{5}$ years. The bremsstrahlung emitting electrons seen with e-ASTROGAM have energies close to the radio synchrotron emitting ones, and lower than those seen in synchrotron X-rays, thus permitting tomographic reconstruction of the magnetic field and electron distributions inside the remnant. Fig. 7 
indicates that e-ASTROGAM has the sensitivity in one year of exposure to detect $\mathrm{CR}$ electrons even for a strong mean magnetic field. The sub-GeV part of the gamma radiation is essential to separate the emission from relativistic electrons and nuclei above $100 \mathrm{MeV}$, so the new data can constrain how electrons and protons are differentially injected into the shock, how large and sometimes highly intermittent magnetic fields build up near the shock, and how the acceleration efficiency and the total CR content of a remnant evolves as the shockwave slows down.

Older remnants often interact with molecular clouds that provide target gas for CRs escaping the remnant [140]. Resolving the diffuse pion emission produced in those clouds against the bright Galactic background is essential to probe the CR spectra that are actually injected into the ISM. Imaging the remnant and shocked clouds both require an angular resolution better than $0.2^{\circ}$ around one $\mathrm{GeV}$ and a sensitivity below $10^{-11} \mathrm{erg}^{-2}$ $\mathrm{s}^{-1}$ above $50 \mathrm{MeV}$ that e-ASTROGAM will achieve. The instrument may also detect line emission from nuclear excitation caused by low-energy CR nuclei, opening a new and unique way to remotely measure the flux and elemental composition of low-energy particles. The comparison with direct composition measurements near the Earth would provide valuable clues as to the type of supernovae that dominate CR production in the Galaxy.

A conservative estimate on the number of SNRs studied in detail by e-ASTROGAM can be obtained starting from the 3FGL catalog of sources in the $100 \mathrm{MeV}-$ $300 \mathrm{GeV}$ energy range based on the first four years of science data from the Fermi mission [7]. This catalog contains 12 SNRs, 9 PWNe, the Cygnus superbubble produced by multiple supernovae [8], as well as 62 additional sources potentially associated to SNRs. Given that e-ASTROGAM will have a comparable or better sensitivity, FoV and observation strategy than Fermi-LAT in the $100 \mathrm{MeV}-1 \mathrm{GeV}$ energy range, and accounting for the measured spectra of SNRs, we expect the detection of at least the same amount of objects of this class in this energy range.

However, e-ASTROGAM will have a much improved sensitivity with respect to Fermi-LAT below $100 \mathrm{MeV}$. This will allow bright SNRs like IC 443, W44, W51C, and W28 to be studied for the first time with good statistics spectra in the very important energy band below the pion-decay bump, which is crucial to make firm conclusions about the hadronic and leptonic contributions to the observed emission. This is very important to constrain the CR acceleration physics.

Another definitive advantage of e-ASTROGAM is its very good sensitivity in the $\mathrm{MeV}$ energy domain, where the ${ }^{44} \mathrm{Ti} 1157 \mathrm{keV}$ line measurements should uncover about 10 new, very young SNRs (of ages up to $500 \mathrm{yr}$ ) presently hidden in highly obscured clouds (see also Sect. II C 2). These objects are thought to accelerate $\mathrm{CR}$ up to $\mathrm{PeV}$ energies and will be prime targets for the Cherenkov Telescope Array (CTA) as well.
2. How do CR fluxes vary with Galactic environments, from passive interstellar clouds to active starburst regions and near the Galactic Center?

The gamma-rays produced by CR nuclei along their interstellar journey can remotely probe the CR flux and spectrum across Galactic spiral arms, inside young stellar clusters and superbubbles, in the central molecular zone, and down to parsec scales inside nearby clouds. The current picture provided by the Fermi-LAT analyses does not provide sufficient resolution to probe theoretical expectations based on spiral distributions of $\mathrm{CR}$ sources and environmental changes in CR transport due to their streaming in the varying Galactic magnetic field 114 or to different levels of interstellar MHD turbulence powered by massive stars and supernovae [71, 84]. With their much improved angular resolution and lower energy band, e-ASTROGAM observations can complement the Fermi-LAT archival data to probe the heterogeneity of the CR population in a variety of Galactic environments, across four decades in momentum around the maximum energy density. The e-ASTROGAM subdegree resolution is essential to map the structured CR emission and avoid confusion with Galactic point sources (Fig. 8). It is also key to resolve starburst regions hosting cocoons of young energetic CRs [8] to reveal the impact on the CR properties (energy and diffusion) of the large level of supersonic turbulence driven by the massive stars. How is the emerging CR spectrum modified by confinement, re-acceleration and enhanced losses in the turbulent medium? Since most CR sources occur in star-forming regions, these questions challenge our global understanding of the early steps of CR propagation and our use of observational diagnostics in the Galaxy at large and in starburst galaxies in particular. The sensitivity and PSF of e-ASTROGAM permit searches for Galactic $\mathrm{CR}$ cocoons other than Cygnus $\mathrm{X}$ and an extensive characterization of the particle emissions across four decades in energy (with Fermi archives and TeV data from CTA and HAWC).

With a sensitivity of $10^{-12} \mathrm{erg} \mathrm{cm}^{-2} \mathrm{~s}^{-1}$ around 100 $\mathrm{MeV}$, e-ASTROGAM can also detect the pion bump from CRs in the Large Magellanic Cloud (LMC), in particular in the star-forming regions of 30 Doradus and N11 [16], and in nearby starburst galaxies [4]. Such observations will provide insight on the $\mathrm{CR}$ in external galaxies at energies relevant for the physics of their ISM. The detection of the pion bump is crucial to disentangle the CR origin of the emission from other sources such as pulsars.

\section{Where are the low-energy CRs and how do they penetrate dense clouds?}

Locally, the CR energy density is dominated by sub$\mathrm{GeV}$ and $\mathrm{GeV}$ protons. It is comparable to the energy densities of the interstellar gas, magnetic field, and stellar radiation. Low-energy cosmic rays (LECRs) influ- 

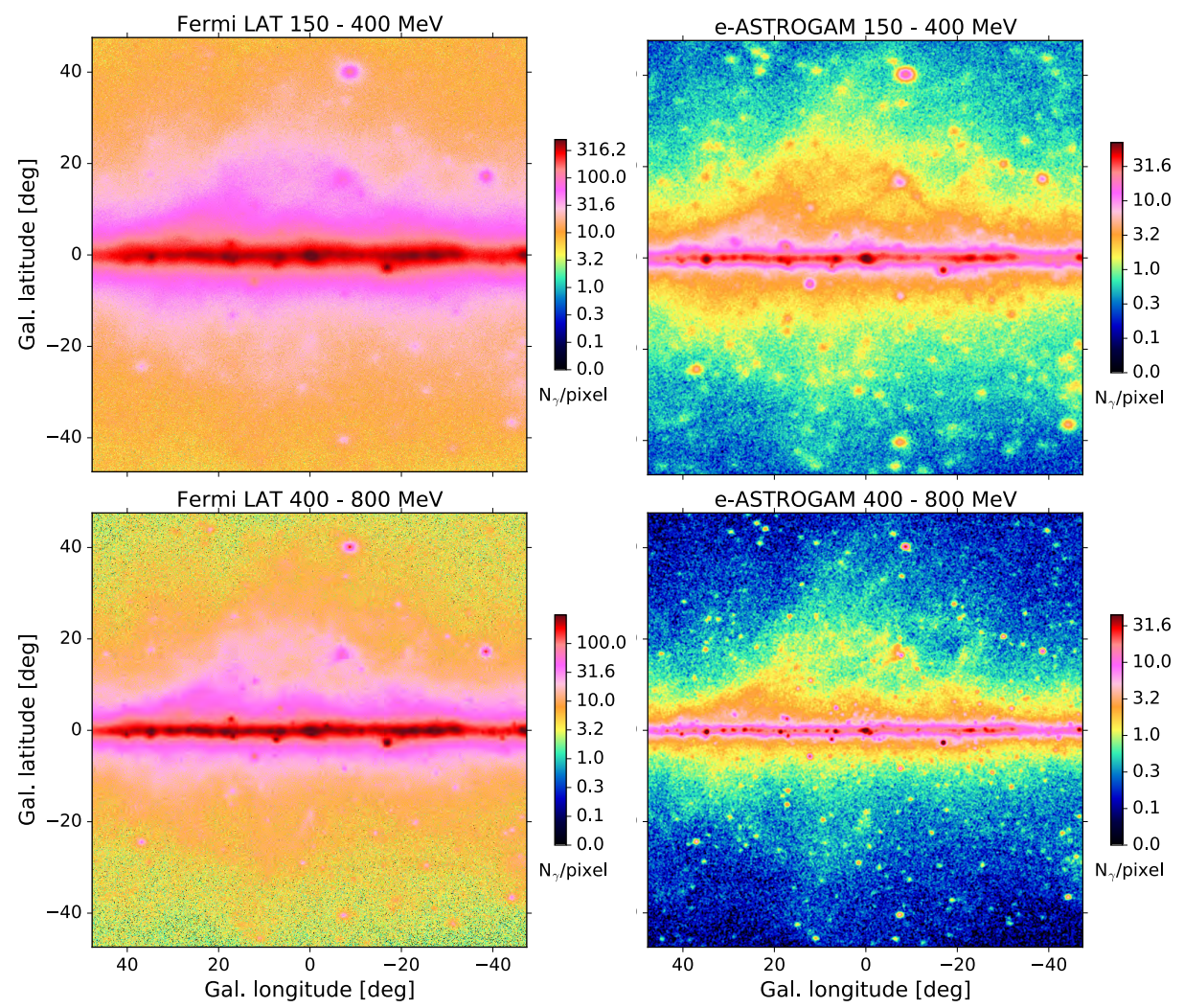

FIG. 8. Imaging the inner Galaxy region: simulated performance of e-ASTROGAM in one year of effective exposure (right panels) compared to 8 years of Fermi (left panels) for the energy ranges $150 \mathrm{MeV}-400 \mathrm{MeV}$ (top) and $400 \mathrm{MeV}-800 \mathrm{MeV}$ (bottom).

ence galactic evolution by changing the thermodynamical state (pressure, heat) and chemical evolution (via the ionization rate) of the dense clouds that lead to star formation. They also provide critical pressure support in starburst regions to launch galactic winds into Galaxy halos [115. Yet, our knowledge of the production pathways and transport properties of LECRs is very rudimentary in our Galaxy, and even more so in the conditions of merger/starburst galaxies. In the Local Bubble, the pronounced break in CR momentum implied near one $\mathrm{GeV}$ by the Voyager and Fermi LAT data suggests that LECRs are advected off the plane by a local Galactic wind [71, 122. Beyond Voyager, indirect measures of the LECR flux at the low, ionizing, energies are uncertain by several orders of magnitude, even in the local ISM. Whereas multi-GeV CRs appear to penetrate deeply and rather uniformly into molecular clouds, molecular line observations suggest strong spatial variations in the ionization induced by LECRs [75].

The energy band and performance of e-ASTROGAM are well suited to probe the distribution of LECRs in different Galactic environments, both from the bremsstrahlung radiation of low-energy electrons and the pion bump from low-energy nuclei. Another long-awaited goal is to test the concentration and exclusion processes that govern the penetration of CRs into dense clouds, which are predicted to leave an energy-dependent signa- ture below $1 \mathrm{GeV}$ 127.

Inelastic collisions of LECRs with interstellar gas should produce a rich spectrum of gamma-ray lines between 0.3 and $10 \mathrm{MeV}$ [27]. Spectroscopic observations of these lines with e-ASTROGAM are the only direct way to detect these elusive particles, to measure their energy density in and out of dense clouds, and to measure the production rate of light elements $(\mathrm{Li}, \mathrm{Be}$, and $\mathrm{B}$ ) resulting from their interactions with gas. Fig. 9 shows that e-ASTROGAM should allow a firm detection of $\gamma$-ray line complexes from the inner Galaxy with a total flux of $\sim 2 \times 10^{-4} \mathrm{~cm}^{-2} \mathrm{~s}^{-1} \mathrm{sr}^{-1}$ in the (0.3-10) MeV band, and possibly also from superbubbles, hypernovae, and active star forming regions.

\section{The origin and energy content of Galactic wind and Fermi bubbles}

There is increasing evidence, observationally (e.g. 47, 87]) and theoretically, for the emergence from the inner $200 \mathrm{pc}$ of the Galaxy of a Galactic wind flowing to large height $(\sim 10 \mathrm{kpc})$ into the halo and partly accelerated by the pressure gradient supplied by CRs [37, 57. The relation with the Fermi Bubbles seen in gamma rays and possibly in microwaves and polarized radio waves is unclear despite their biconical structure and the finding of 


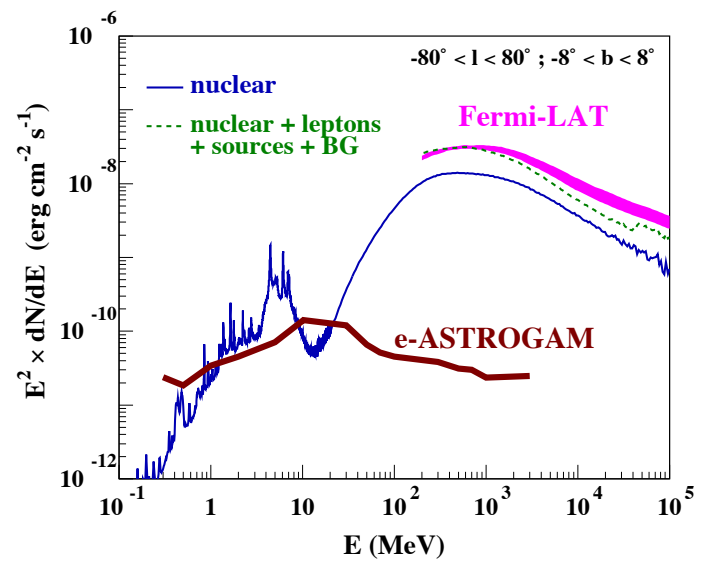

FIG. 9. Predicted gamma-ray emission due to nuclear interactions of CRs in the inner Galaxy. The gamma-ray line emission below $10 \mathrm{MeV}$ is due to LECRs, whose properties in the ISM have been adjusted such that the mean CR ionization rate deduced from $\mathrm{H}_{3}^{+}$observations and the Fermi-LAT data (magenta band) at $1 \mathrm{GeV}$ are simultaneously reproduced (adapted from [27]). The 1-year sensitivity of e-ASTROGAM (for Galactic background) is superimposed.

gas at large velocities $(>900 \mathrm{~km} / \mathrm{s})$ in their direction [59]. The Bubbles may be the few-Myr-old relics of past accretion-driven outflow(s) from $\mathrm{Sgr} \mathrm{A}^{*}$ or the CR-driven Galactic wind powered by the starburst activity in the central nucleus. They are filled with relativistic particles of unknown nature (electrons or nuclei) and origin. The bubble shapes near the Galactic disc and their spectrum below $0.5 \mathrm{GeV}$ are quite uncertain because of the large confusion with Galactic foregrounds [12]. The improved angular resolution of e-ASTROGAM will reveal the geometry and sub-GeV spectrum of the Bubbles down to their base to help identify the dominant particles, study particle ageing in the outflow, distinguish between impulsive and wind models, and estimate the total power expelled from the modest nucleus of our Galaxy.

\section{Antimatter and WIMP Dark Matter}

The detection of a bright gamma-ray $e^{+} e^{-}$annihilation line at $511 \mathrm{keV}$ places strong constraints in many emission models for high-energy astrophysical sources. The detection of $e^{+} e^{-}$annihilation radiation can thus serve as an important and unambiguous calorimeter, e.g., to track supernova nucleosynthetic activity or constrain the presence of dark matter at the center of our Galaxy. e-ASTROGAM will have significantly better sensitivity than INTEGRAL/SPI in the $511 \mathrm{keV}$ line (TableVP. It would represent a major step forward, for example, in answering the many questions posed by INTEGRAL's detection of spatially extended $e^{+} e^{-}$annihilation in our Galaxy. Note also that e-ASTROGAM's energy coverage is ideally suited (compared to Fermi) to capture the radiation from decaying pions and anti-proton annihilation, especially if the relevant sources are cosmological and redshifted. By either resolving the extragalactic gammaray background at $\sim 1-100 \mathrm{MeV}$ or constraining its angular anisotropy, e-ASTROGAM could thus directly detect or, at least, significantly improve on COMPTEL constraints for anti-matter domains in the Universe left over from the Big Bang and the process of baryogenesis.

The theoretical paradigm about dark matter (DM) most popular today is motivated by the so-called WIMP miracle (i.e., the fact that we can explain the missing mass with just one more particle, and without the need for new interactions). Such a Weakly Interacting Massive Particle, or WIMP, is present in many extensions of the Standard Model (SM) of particle physics (e.g. the neutralinos in supersymmetry, or the Kaluza-Klein states in theories with extra dimensions, see e.g. 49 ). To account for the missing matter, a WIMP that comes from an equilibrium state in the early Universe should have mass $\mathrm{O}\left(100 \mathrm{GeV} / c^{2}\right)$, and a self-annihilation cross section $\sigma$ and a typical velocity $v$ such that $\left\langle\sigma v>\sim 3 \times 10^{-26}\right.$ $\mathrm{cm}^{3} / \mathrm{s}$ (this value is a kind of "thermal benchmark). The annihilation of pairs of WIMPs in high-density DM regions could result in an excess of photons and antimatter coming mainly from the hadronic cascades; for $m_{\text {WIMP }}<100 \mathrm{GeV} / c^{2}$ the bulk of photons is expected below $1 \mathrm{GeV}$ [10]. Monochromatic lines from 2-body final states (either of which being a photon, or both) are not excluded.

In final states characterized by a photon continuum or by $\gamma$-ray lines, the e-ASTROGAM sensitivity for the detection of DM is complementary to Fermi and CTA because it covers with larger sensitivity the low-mass interval. Moreover, the possibility of Fermi (and in the future of CTA) to detect DM requires that these experiments demonstrate that there is an excess of photons. Modeling the photon background from astrophysical sources is where e-ASTROGAM is fundamental, since the $\mathrm{MeV} / \mathrm{GeV}$ range is probably the most constraining for the SED of astrophysical sources. In addition, eASTROGAM can constrain the signal from nearby pulsars.

The region of the Galactic Center (GC) is expected to host the highest density of DM in the vicinity of the Earth, but the many astrophysical processes at work in the crowded inner Galaxy make it extremely difficult to disentangle the possible DM signal from conventional emissions [112]. e-ASTROGAM will improve our understanding of the origin of particles in the inner Galaxy, therefore reducing the uncertainties associated to DM searches.

Other targets for the search of DM are dwarf spheroidal galaxies (dSphs, [144]), whose otherwise mysterious dynamics can be simply explained if they are highly DMdominated $\left(M / L \sim 10^{3} M_{\odot} / L_{\odot}\right)$. dSph allows an almost background free observation, since they are not expected to be $\gamma$-ray emitters unless a sizable WIMP pair annihilation takes place. There are currently about 30 known dSphs, with new objects in this class being discovered. 
For a low WIMP mass, e-ASTROGAM will have a discovery potential comparable to Fermi-LAT - stronger if the DM mass is on the few $\mathrm{GeV}$ scale.

In some extensions of the $\mathrm{SM}, \mathrm{DM}$ is on the $\mathrm{MeV}$ scale. Theories predict a stable relic particle, in thermal equilibrium during the early Universe, with mass between 1 and $100 \mathrm{MeV}$ 35. Such models attracted some interest about a decade ago because they would naturally explain the 511-keV emission line toward the galactic bulge [36, 126]. In this case e-ASTROGAM could make a direct detection or provide constraining limits 34. e-ASTROGAM outperforms Fermi and has a sensitivity much better than the thermal benchmark for masses below $5 \mathrm{GeV}$ for the search of a continuum signal in dSphs and below $200 \mathrm{MeV}$ for line searches 14 .

Besides the popular paradigms outlined above, other scenarios are discussed in the literature for DM, in which e-ASTROGAM has the best sensitivity, in particular:

- Monochromatic photons at MeV energies may result from DM annihilation to quarkonium [120], as well as step-like features from the decay $b \rightarrow s+\gamma$ or $b^{\prime} \rightarrow b+\gamma$, where $b^{\prime}$ is a hypothetical 4 th generation quark 28. Another possibility is the decay of DM candidates like the gravitino, which has motivated line searches with the Fermi Large Area Telescope down to energies of $100 \mathrm{MeV}$ [21]. It was also pointed out that for DM lighter than around $100 \mathrm{MeV}$, the only kinematically accessible nonleptonic states are photons and neutral pions, leading to clear gamma-ray signatures to look for 34 . Due to the gap in sensitivity at the $\mathrm{MeV}$, very weak limits on DM signals exist in this range [56].

- A new class of potential smoking-gun signatures for DM signals in the range $10 \mathrm{MeV}-100 \mathrm{MeV}$ is pointed out in 38 and involve transitions between meson states and, in their simplest realization, do not require any new physics (beyond, obviously, the DM particle itself) but inevitably arise in certain kinematical situations for GeV-scale DM annihilating or decaying to heavy quarks. Unlike direct detection or collider experiments, these signatures are thus very sensitive to DM coupling with third or second generation quarks.

- Axion-Like-Particle DM (of extremely low mass); this is discussed in the observatory science (Sect. II D.

e-ASTROGAM can also shed new light on dark matter by the study of antimatter - an excess of antimatter is expected from DM annihilation. The case of signals from excesses of antimatter is particularly intriguing: the presently measured flux of mildly relativistic cosmic rays (anti-electrons in particular) cannot be explained on the basis of present knowledge, and the data show an excess with respect to known astrophysical sources (PAMELA, AMS02; see 101 for a review). Is this excess due to presently unknown sources, e.g. as yet unknown pulsars or past activity of the GC [42, 109], or are we detecting evidence of new physics at the fundamental scale? This question can be answered by observations of nearby pulsars with e-ASTROGAM.

Finally an improved angular resolution with respect to AGILE and Fermi-LAT in the inner Galaxy region and in regions closer to Earth in the $5 \mathrm{MeV}-100 \mathrm{MeV}$ energy range can disentangle the possible contributions from the diffuse background, from point sources, and other possible emitters. Overall, a large class of spectral features in the $\mathrm{MeV}-\mathrm{GeV}$ range can result in indications for WIMP DM particles, or significantly reduce the astrophysical background uncertainties to identify genuine DM signatures in VHE photon spectra 38.

\section{Nucleosynthesis and the chemical evolution of our Galaxy}

The origins of the cosmic atomic nuclei and their variety is one of the main themes of astrophysical research, and has been studied through nuclear gamma-ray emission for several decades. The Compton Gamma Ray Observatory (CGRO) provided the first sky survey of nuclear emission from cosmic sources [55, 123, INTEGRAL added high-resolution spectroscopy [52]. From these missions, however, only the brightest sources of their class have been seen, and a deeper survey as proposed with eASTROGAM will address fundamental issues in nuclear astrophysics, exploring the variability of nuclear emission from supernovae (SNe), localized stellar groups, and other transients related to compact stars and nuclear processes therein. Key science goals of e-ASTROGAM focus on the astrophysics of $\mathrm{SNe}$, for which a validated and selfconsistent model has not yet been established, neither for thermonuclear supernovae (SN Ia) nor for core collapse supernovae ( $\mathrm{SN} \mathrm{Ib/c} \mathrm{and} \mathrm{II).}$

1. What are the progenitor system(s) and explosion mechanism(s) of thermonuclear SNe? Can we use SN Ia for precision cosmology?

SN Ia are the outcome of a thermonuclear burning front that sweeps a carbon/oxygen white dwarf in a close binary system. But exactly how the ignition conditions are obtained, and on which white dwarfs, and more so how the thermonuclear runaway proceeds through the white dwarf and turns it into a variety of isotopes that are ejected, are all questions that are subject to considerable debate (e.g. 73, 74 and references therein). It seems that several candidate evolutionary channels may all contribute, from the double degenerate variant of merging white dwarf binaries disrupting one of the dwarfs through tidal forces or a hard collision, to a variety of single degenerate models where accretion of material from a companion star may lead to either the white dwarf reaching the critical Chandrasekhar mass stability 


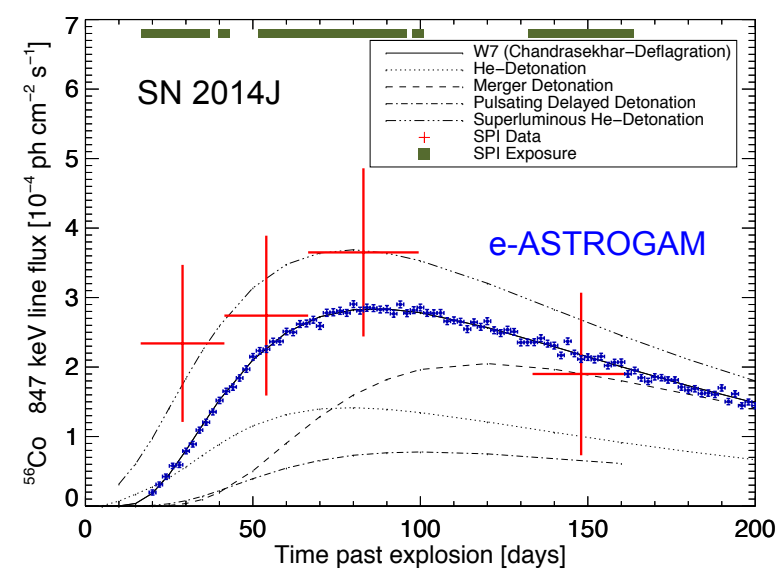

FIG. 10. The evolution of the $847 \mathrm{keV}$ line brightness from ${ }^{56}$ Co decay reflects how radioactive energy is deposited within a supernova to make it shine. Here, INTEGRAL data from the Type Ia supernova SN 2014J (adapted from Fig. 4 in Ref. [54, red data points) are compared to a family of candidate models [137. A simulation of the response to a time evolution such as in the W7 model [99] shows that the sensitivity improvement by e-ASTROGAM (blue points) will lead to breakthrough science.

limit, or be ignited earlier through a surface explosion from a helium flash.

Such uncertainties are troublesome for cosmology since the use of SN Ia as standard candles depends on an empirical relationship between the shape and the maximum of the light curve [110]. Although useful up to now, in view of the development of precision cosmology, a better, astrophysically supported understanding of thermonuclear SNe, as well as their evolutionary effects at large distances and low metallicities, are mandatory. The brightness-decline relation [110] is closely related to the mass of synthesized ${ }^{56} \mathrm{Ni}$, and factors like the progenitor evolution, ignition density, flame propagation, mixing during the burning, completeness of burning in outer, expanding regions, all lead to different amounts of ${ }^{56} \mathrm{Ni}$, which is measured directly through gamma-ray lines. On the other hand, radiation transport from radioactivity to optical light and their spectra depend on complex atomic line transitions in the expanding supernova as well as total mass burned, the amount and distribution of radioactive nickel and intermediate mass elements, all of which must combine in quite a tight way to reproduce the observations 82 , 148. Some of these factors depend on the evolution of the white dwarf prior to the explosion and cast some doubt on the use of SN Ia as high precision distance indicators. It is thus of critical importance to disentangle the role of these factors to understand the limits of the Phillips [110 relation.

With SN2014J, for the first time a SN Ia occurred close enough for current generation $\gamma$ ray telescopes, at $3.5 \mathrm{Mpc}$ in the starburst galaxy M82. INTEGRAL could detect the long awaited gamma-ray signatures of the thermonuclear runaway, through the early emission from the decay of ${ }^{56} \mathrm{Ni}$ (mean lifetime $\tau \simeq 8.8$ days) about 20 days after the explosion, and the main gamma-ray lines at 847, 1238, and $511 \mathrm{keV}$ from the decay of ${ }^{56} \mathrm{Co}$ ( $\tau \simeq 111$ days). These data suggested either a surface explosion or some unusual morphology of the runaway [44, 45, 53, 54, 76], either case in stark contrast to the conventional Chandrasekhar model. Clearly, the glimpse offered by SN2014J observations underlines the importance of gamma-ray line diagnostics in these systems and emphasize that more and better observations hold the key to a deeper understanding of how the thermonuclear explosion of a white dwarf star unfolds.

e-ASTROGAM will achieve a major gain in sensitivity compared to INTEGRAL for the main gamma-ray lines arising from ${ }^{56} \mathrm{Ni}$ and ${ }^{56} \mathrm{Co}$ decays. Thus with the expected sensitivity of $3.5 \times 10^{-6} \mathrm{ph} \mathrm{cm}^{-2} \mathrm{~s}^{-1}$ for the $847 \mathrm{keV}$ line in $1 \mathrm{Ms}$ of integration time (see Table VP, e-ASTROGAM will detect $(10 \pm 3)$ SNe Ia in 3 years of nominal mission lifetime, up to a distance of $\sim 35 \mathrm{Mpc}$ (for the brightest $\mathrm{SNe}$ ). As illustrated in Fig. 10, e-ASTROGAM will provide much better data than we have now with INTEGRAL for SN 2014J from similarly nearby events. These data will allow us to probe the explosion mechanism in detail, and compare with astrophysical models for each event to better understand the progenitor system(s) and the thermonuclear explosion process.

Gamma rays escaping from supernovae contribute to the diffuse background in the $\mathrm{MeV}$ range. The contribution from Type Ia supernovae dominates, potentially providing a significant fraction of the observed flux in the $\mathrm{MeV}$ band. (see, e.g., [121]).

2. How do core-collapse supernovae (CCSNe) explode? What is the recent history of CCSNe in the Milky Way?

Similar to SN Ia, core collapse physics is also not well understood in terms of an astrophysical model. But these events are more common, being the end states of the evolution of massive stars, and are key to understanding the diversity of elements in the universe. Also here, deviations from spherical symmetry are the rule. The goal is to explain a tremendous variety of core collapse events, e.g. electron capture supernovae such as the Crab, clumpy explosions such as Cas A, collapsars that appear as GRB sources and produce stellar mass black holes, superluminous supernovae that may be powered entirely differently by magnetar rotational energy, or pair instability supernovae that create huge amounts of radioactive ${ }^{56} \mathrm{Ni}$.

Stellar rotation is known to exist but is complicated to track in its effects on stellar evolution, yet important for many of the above outcomes: nucleosynthesis, pre-supernova structure, core collapse. Measuring nucleosynthesis products such as ${ }^{56} \mathrm{Ni},{ }^{56} \mathrm{Co}$ and ${ }^{44} \mathrm{Ti}$ is one of the more direct ways to extract information on the inner processes triggering the explosion near the newly forming compact stellar remnant (e.g., 70]) - other observables 
are indirect, and mostly reflect interactions within the envelope, or with circumstellar, pre-explosively ejected, or ambient gas. e-ASTROGAM will detect the signatures of ${ }^{56} \mathrm{Ni}$ and ${ }^{56} \mathrm{Co}$ decay from several CCSNe in nearby galaxies. Comparing $\gamma$-ray characteristics of different classes of CCSNe, possibly including the pair instability SNe with their order of magnitude higher ${ }^{56} \mathrm{Ni}$ production [61, will probe potentially large variations in their progenitors and offer a direct view of their central engines.

With a gain in sensitivity for the ${ }^{44} \mathrm{Ti}$ line at $1157 \mathrm{keV}$ by a factor of 27 compared to INTEGRAL/SPI (see Table V), e-ASTROGAM should also detect the radioactive emission from ${ }^{44} \mathrm{Ti}$ ( $\tau \simeq 87$ years) from most of the young (age $\lesssim 500 \mathrm{yr}$ ) SNRs in the Milky Way, thus uncovering about 10 new SNRs in the Galaxy, as well as the youngest SNR(s) in the LMC. Among the youngest Galactic SNRs, only Cassiopeia A has been firmly detected in ${ }^{44} \mathrm{Ti}$ surveys carried out up to now [139, which is surprising in view of an otherwise inferred rate of one CCSN every 50 years in our Galaxy. e-ASTROGAM will measure in particular the amounts of ${ }^{44} \mathrm{Ti}$ in the SNRs G1.9+0.3, Tycho and SN 1987A (LMC), which are currently disputed in the literature (see, e.g., [139]). Besides giving new insights on the dynamics of core collapse at the mass cut, e-ASTROGAM observations of young SNR will be very important for cosmic-ray physics. Indeed, only young SNRs can accelerate CR up to PeV energies and above, and the identification of new Pevatrons would be invaluable when the Cherenkov Telescope Array (CTA) will be fully operational.

\section{Nova explosions}

e-ASTROGAM will also contribute to the study of novae, which are responsible for the enrichment of the Galaxy in some species and for the peculiar isotopic signatures found in some pre-solar meteoritic grains [79]. Emission is expected 68, 72 from positron-electron annihilation ( $\mathrm{e}^{+}$being emitted by the $\beta^{+}$-unstable shortlived isotopes ${ }^{13} \mathrm{~N}$ and ${ }^{18} \mathrm{~F}$ ), and from the decay of the medium-lived isotopes ${ }^{22} \mathrm{Na}$, which is produced in $\mathrm{ONe}$ novae, and ${ }^{7} \mathrm{Be}$, produced in $\mathrm{CO}$ novae. The first type of emission consists of a $511 \mathrm{keV}$ line plus a continuum between about 20 and $511 \mathrm{keV}$, whereas the second, longlasting emission consists of two $\gamma$-ray lines at $1275 \mathrm{keV}$ $\left({ }^{22} \mathrm{Na}\right)$ and $478 \mathrm{keV}\left({ }^{7} \mathrm{Be}\right)$. Both provide a direct insight on the amount of radioactive nuclei in the expanding nova envelope, an information only obtainable through the observation of $\gamma$-rays. The large FoV of e-ASTROGAM is crucial for these observations, since the $511 \mathrm{keV}$ line emission happens before the nova is discovered optically, preventing pointed observations. The detectability distance with e-ASTROGAM is around $3 \mathrm{kpc}$ for both the 478 and $1275 \mathrm{keV}$ line, and $4-6$ novae at distance $D<3 \mathrm{kpc}$ are expected in the 3 years of nominal mission lifetime.

e-ASTROGAM will also help to disentangle the current puzzle posed by the detection of novae at $\mathrm{GeV}$ en- ergies with Fermi-LAT [11, 43]. Some novae have been identified theoretically as sites of particle acceleration, in the shocks within the ejecta and/or between the ejecta and the circumstellar matter, making them responsible for a fraction of the Galactic cosmic rays [132. These "miniature supernovae" are key systems to study the time dependence of diffusive shock acceleration of CR. An important consequence of the production of high-energy particles is that photons with energies higher than about $100 \mathrm{MeV}$ are emitted, both via neutral pion decay and IC processes. With the e-ASTROGAM sensitivity, it will be possible to disentangle the origin of this high-energy emission, hadronic and/or leptonic, and thus understand better the properties of the nova ejecta and the shocks.

\section{How are cosmic isotopes created in stars and distributed in the interstellar medium?}

The cycle of matter proceeds from the formation of stars through nuclear fusion reactions within stars during their evolution, towards the ejection of stellar debris into interstellar space in winds and SN explosions. Interstellar gas, enriched with some newly produced nuclei, eventually cools down to form new stars, closing and starting the cycle again. The cooling down of hot nucleosynthesis ejecta and their trajectories towards new star formation are particularly hard to constrain through observations. The recycling time scale in the interstellar medium is of the order of tens of Myr, but SNRs can be seen over time scales of few $10^{5}$ yr at most. Long-lived radioactive gamma-ray emitters ${ }^{26} \mathrm{Al}\left(\tau \simeq 1.0 \times 10^{6}\right.$ yr $)$ and ${ }^{60} \mathrm{Fe}$ $\left(\tau \simeq 3.8 \times 10^{6} \mathrm{yr}\right)$ can trace mixing processes of ejecta into the next generation star forming regions over much longer time, testing, among other things, molecular-cloud lifetime and models for stimulated/triggered star formation.

INTEGRAL/SPI data for the $1809 \mathrm{keV}{ }^{26} \mathrm{Al}$ line suggest that on the global, Galactic scale, superbubbles are key structures in the transport of fresh ejecta towards new star forming regions 86, 87. With its huge increase in sensitivity, e-ASTROGAM will provide a detailed view of the morphology of this emission, with high precision measurements of the line flux from many regions of the Galaxy. Thus, e-ASTROGAM will observe the ${ }^{26} \mathrm{Al}$ radioactivity from dozens of nearby $(\sim \mathrm{kpc})$ stellar objects and associations. In particular, it will measure precisely the amount of ${ }^{26} \mathrm{Al}$ ejected by the Wolf-Rayet star WR11 in the $\gamma^{2}$-Velorum binary system (expected line flux of $\sim 10^{-5} \mathrm{ph} \mathrm{cm}^{-2} \mathrm{~s}^{-1}$ ), thus providing a unique calibration of the ${ }^{26} \mathrm{Al}$ production during the Wolf-Rayet phase of a massive star. e-ASTROGAM has also the capability of detecting ${ }^{26} \mathrm{Al}$ emission from the LMC (expected line flux of $\sim 10^{-6} \mathrm{ph} \mathrm{cm}^{-2} \mathrm{~s}^{-1}$ ), thus providing new insight into stellar nucleosynthesis outside the Milky Way.

For the first time, e-ASTROGAM will provide the sensitivity needed to establish the Galactic ${ }^{60} \mathrm{Fe}$ emission and build an accurate map of the ${ }^{60} \mathrm{Fe}$ flux in the Milky Way, enabling its comparison with the ${ }^{26} \mathrm{Al}$ map 
to gain insight into the stellar progenitors of both radioisotopes. In particular, measuring $\gamma$-ray line ratios for specific massive-star groups will constrain ${ }^{60} \mathrm{Fe}$ production in massive stars beyond $\sim 40 M_{\odot}$, which directly relates to stellar rotation and uncertain convective-layer evolution in massive star interiors [89].

\section{Observatory science in the $\mathrm{MeV}-\mathrm{GeV}$ domain}

During the first phase e-ASTROGAM will collect data especially for the core science topics, as described above. However, given the very large sky coverage and the accumulated exposure, a very large number of sources can be detected and monitored. e-ASTROGAM has the capability of studying thousands of sources both Galactic and extragalactic of which many are expected to be new detections. Therefore, a very large community of astronomical users will benefit from e-ASTROGAM data available for multifrequency studies through a Guest Investigator programme managed by ESA.

e-ASTROGAM will detect with highly improved sensitivity in the $\mathrm{MeV}-\mathrm{GeV}$ domain phenomena characterized by: (1) rapid and very rapid variability timescales (subsecond, second, minutes, hours), and (2) steady sources. The e-ASTROGAM sensitivity to pointlike sources varying on timescales of seconds (for GRBs) and minuteshours-days (compact objects, novae, magnetars, blazars) will provide unique information about outstanding physical processes including jet processes, shock accelerations and magnetic field reconnection. The study of steady sources (diffuse emission, pulsars, PWNe, SNRs, extragalactic background) will provide a detailed diagnostic of fundamental processes that operate in quasi-stable regimes. The e-ASTROGAM Observatory science program will emphasize multifrequency response to both variable and steady sources in a decade that will benefit from the operations of many other observatories planned to be operative in the 2030s, that include LIGOVirgo-GEO600-KAGRA, SKA, ALMA, E-ELT, LSST, ATHENA, CTA and possibly e-LISA. e-ASTROGAM will provide unique data for multifrequency science, triggering other instruments and reacting rapidly to transient detections.

We summarize here the most relevant classes of phenomena or sources in addition to the e-ASTROGAM "core science" topics.

- Diffuse Galactic gamma-ray background, for which e-ASTROGAM is in a position to determine the underlying cosmic ray population and spatial and spectral variations across the Galaxy.

- Pulsars and millisecond pulsars both isolated and in binaries, whose (pulsed or unpulsed) emission will be observable in a spectral range rich in information to discriminate between particle acceleration models.
- Pulsar wind nebulae, a product of the interaction between shocked relativistic pulsar winds and the ISM, for which e-ASTROGAM will obtain crucial data on particle acceleration and propagation.

- Magnetars, enigmatic and strongly variable compact stars characterized by very strong magnetic fields that exhibit special phenomena exclusively in the $\mathrm{MeV}$ energy range.

- Galactic compact binaries, including white dwarfs, neutron stars and stellar mass black holes whose spectral transitions and outbursts in the $\mathrm{MeV}$ range will be systematically monitored by eASTROGAM.

- Novae, that in addition to line emission in the $\mathrm{MeV}$ range can also be studied for their surprising and poorly understood $\gamma$-ray emission up to hundreds of $\mathrm{MeV}$, a product of shock interaction within the nova ejecta and/or of the nova ejecta with the circumstellar matter (see Sect. II C 3).

- Massive binary stars with colliding winds of the Eta-Carinae type, whose MHD shocks are predicted to produce particle acceleration and $\gamma$ ray emission, a topic of great interest and yet unsettled.

- Interstellar shocks, such as the Cygnus cocoon showing the existence of particle acceleration over large distances in the ISM, for which the spectral and angular resolution of e-ASTROGAM will be unique.

- Blazar population studies in the $\mathrm{MeV}$ range, to be obtained by the detection capability of thousands of sources by e-ASTROGAM.

- Studies of the propagation of $\gamma$-rays over cosmological distances, for which the attenuation is predicted to be negligible in standard QED - effects of absorption might indicate new physics at work, possibly the existence of axion-like-particles (ALPs) coupling to gamma-rays [48. ALPs $a$ are spin-0, neutral and very light particles predicted by many extensions of the SM [77, coupling to two photons through an amplitude $g_{a \gamma} \mathbf{E} \cdot \mathbf{B}$ (presently bound by $g_{a \gamma}<0.66 \cdot 10^{-10} \mathrm{GeV}^{-1}$ [23, 116] for a wide mass range.) In a photon beam emitted by a far-away source (blazar, GRB), $\gamma \rightarrow a$ and $a \rightarrow \gamma$ conversions can take place, resulting in photon-ALP oscillations. These may show up in the e-ASTROGAM energy spectrum about $E_{L}=2.56 \cdot 10^{21}(\mathrm{~m} / \mathrm{eV})^{2} \xi^{-1} \mathrm{GeV}$ for an ALP mass $3.42 \cdot 10^{-13} \xi^{1 / 2} \mathrm{eV}<m<3.42 \cdot 10^{-11} \xi^{1 / 2} \mathrm{eV}$, where $\xi \equiv\left(g_{a \gamma} 10^{11} \mathrm{GeV}\right)(B / \mathrm{nG})[48$. For a $100 \%$ polarized beam the amplitude of the fluctuations can be twice then for an unpolarized beam [62, 149. CCSNe can also be a source of ALPs, produced 
in the few seconds after the explosion inside the core by Primakoff effect, and reconverted to photons of the same energy (peaking at $\sim 50 \mathrm{MeV}$ ) in the Milky Way magnetic field. The arrival time of these photons would be the same as for neutrinos, providing a clear signature; e-ASTROGAM would have a sensitivity better than Fermi-LAT 94 and access to much smaller mass/coupling values than dedicated laboratory experiments.

- Solar flares and contribution to "Space Weather", that will be studied with unprecedented line emission and continuum capability for theoretical modeling as well as fast reaction for alerts.

The Sun is an efficient particle accelerator during flares. A possible mechanism requires that ions and electrons are accelerated by the release of energy during magnetic reconnection in the upper corona; they can then propagate into the chromosphere where they interact to produce a strong $\mathrm{X} / \gamma$-ray bremsstrahlung continuum, nuclear lines (through nuclear interactions with the solar atmosphere: prompt deexcitation lines from e.g. ${ }^{12} \mathrm{C}^{*}$ at $4.44 \mathrm{MeV}$ and ${ }^{16} \mathrm{O}^{*}$ at $6.2 \mathrm{MeV}$ but also a neutron capture line at $2.2 \mathrm{MeV}$ from $n \mathrm{H} \rightarrow \mathrm{D}+2.2 \mathrm{MeV}$ photons), and pion-decay components.

After a solar flare, the Sun is predicted to produce $\gamma$-ray emission through radioactive decays (e.g. lines at 847 and $1434 \mathrm{keV}$ from the decay of ${ }^{56} \mathrm{Co}$ and ${ }^{52} \mathrm{Mn}$, respectively). The detection of these lines would provide additional information on energy spectra and composition of flare-accelerated particles, and on mixing processes in the solar atmosphere. e-ASTROGAM can study for the first time solar-flare radiation from $300 \mathrm{keV}$ to $>100$ $\mathrm{MeV}$.

To summarize, the continuum and line sensitivity of e-ASTROGAM in the (0.3-100) $\mathrm{MeV}$ energy range will provide a diagnostic for acceleration mechanisms with simultaneous information at $\mathrm{GeV}$ energies. MeV polarization will add important information on the in-situ magnetic properties.

The solar emission produced by interactions of CRs with the surface and the heliosphere [97, 103, 104 will be also observed. This will allow precise studies on the CRs and their propagation very close to the Sun.

- Terrestrial Gamma-Ray Flashes, an atmospheric phenomenon with possible environmental impact for which e-ASTROGAM can provide continuous monitoring (including the $511-\mathrm{keV}$ line detection).

Atmospheric lightning and thunderstorms can produce particle acceleration in TGFs up to the GeV; the overall expected TGF detection rate by eASTROGAM is of about 60 TGFs per day, improving by a factor of $30 \mathrm{Fermi-LAT}$. Observations in the Compton and pair production regime will provide TGF imaging in the $\mathrm{MeV}-100 \mathrm{MeV}$ range. The optimal sensitivity of e-ASTROGAM in the $\mathrm{MeV}-\mathrm{GeV}$ range will clarify the physics and the many implications of this puzzling atmospheric phenomenon.

\section{SCIENTIFIC REQUIREMENTS}

e-ASTROGAM's requirements to achieve its core science objectives, such as the angular and energy resolution, the field of view, the continuum and line sensitivity, the polarization sensitivity, and the timing accuracy, are summarized in Table I.

- The very large spectral band covered by the telescope in the standard gamma-ray acquisition mode will give a complete view of the main nonthermal processes at work in a given astrophysical object, for the first time with a single instrument. The e-ASTROGAM energy band includes the $511 \mathrm{keV}$ line from $e^{+} e^{-}$annihilation, the nuclear de-excitation lines, the characteristic spectral bump from pion decay, the typical domains of nonthermal electron bremsstrahlung and IC emission, as well as the high-energy range of synchrotron radiation in sources with high magnetic field $(B \gtrsim 1 \mathrm{G})$. The designed wide energy band is particularly important for the study of blazars, GRBs, Galactic compact binaries, pulsars, as well as the physics of CRs in SNRs and in the ISM.

- The large energy band covered by the Calorimeter in the burst search mode of data acquisition (Sect. IV A 5) is primarily designed for the triggering and study of GRBs. It is also well adapted to the broadband emissions of TGFs and solar flares.

- The wide field of view of the telescope is especially important to enable the measurement of source flux variability over a wide range of timescales both for a-priori chosen sources and in serendipitous observations. Coupled with the scanning mode of operation, this capability enables continuous monitoring of source fluxes that will greatly increase the chances of detecting correlated flux variability with other wavelengths. The designed wide field of view is particularly important for the study of blazars, GRBs, Galactic compact objects, supernovae, novae, and extended emissions in the Milky Way (CRs, radioactivity). It will also enable, for example, searches of periodicity and orbital modulation in binary systems.

- One of the main requirements of e-ASTROGAM is to improve dramatically the detection sensitivity 
TABLE I. e-ASTROGAM scientific requirements.

\begin{tabular}{|c|c|}
\hline Parameter & Value \\
\hline Energy bands: & $\begin{array}{ll}0.3 \mathrm{MeV}-3 \mathrm{GeV} & \text { (Gamma-ray imager: Tracker + Calorimeter) } \\
30 \mathrm{keV}-200 \mathrm{MeV} & \text { (Calorimeter burst search) }\end{array}$ \\
\hline $\begin{array}{l}\text { Gamma-ray imager FOV } \\
\text { (at } 100 \mathrm{MeV} \text { ) }\end{array}$ & $\geq 2.5 \mathrm{sr}$ \\
\hline $\begin{array}{l}\text { Gamma-ray imager } \\
\text { Continuum flux sensitivity } \\
\text { at } 3 \sigma \text { confidence level }\end{array}$ & $\begin{array}{l}<2 \times 10^{-5} \mathrm{MeV} \mathrm{cm}^{-2} \mathrm{~s}^{-1} \text { at } 1 \mathrm{MeV}\left(T_{\text {obs }}=10^{6} \mathrm{~s} \text { effective observation time }\right) \\
<5 \times 10^{-5} \mathrm{MeV} \mathrm{cm}^{-2} \mathrm{~s}^{-1} \text { at } 10 \mathrm{MeV}\left(T_{\text {obs }}=10^{6} \mathrm{~s} \text {, high-latitude source }\right) \\
<3 \times 10^{-6} \mathrm{MeV} \mathrm{cm}^{-2} \mathrm{~s}^{-1} \text { at } 500 \mathrm{MeV}\left(T_{\text {obs }}=10^{6} \mathrm{~s} \text {, high-latitude source }\right)\end{array}$ \\
\hline $\begin{array}{l}\text { Gamma-ray imager } \\
\text { Line flux sensitivity } \\
\text { at } 3 \sigma \text { confidence level }\end{array}$ & $\begin{array}{l}<5 \times 10^{-6} \mathrm{ph} \mathrm{cm}^{-2} \mathrm{~s}^{-1} \text { for the } 511 \mathrm{keV} \text { line }\left(T_{\text {obs }}=10^{6} \mathrm{~s} \text { effective obs. time }\right) \\
<5 \times 10^{-6} \mathrm{ph} \mathrm{cm}^{-2} \mathrm{~s}^{-1} \text { for the } 847 \mathrm{keV} \text { SN Ia line }\left(T_{\text {obs }}=10^{6} \mathrm{~s}\right) \\
<3 \times 10^{-6} \mathrm{ph} \mathrm{cm}^{-2} \mathrm{~s}^{-1} \text { for the } 4.44 \mathrm{MeV} \text { line from LECRs }\left(T_{\mathrm{obs}}=10^{6} \mathrm{~s}\right)\end{array}$ \\
\hline $\begin{array}{l}\text { Gamma-ray imager angular } \\
\text { resolution }\end{array}$ & $\begin{array}{l}\leq 1.5^{\circ} \text { at } 1 \mathrm{MeV} \quad(\mathrm{FWHM} \text { of the angular resolution measure }) \\
\leq 1.5^{\circ} \text { at } 100 \mathrm{MeV} \quad(68 \% \text { containment radius }) \\
\leq 0.2^{\circ} \text { at } 1 \mathrm{GeV} \quad(68 \% \text { containment radius })\end{array}$ \\
\hline $\begin{array}{l}\text { AC particle background } \\
\text { rejection efficiency }\end{array}$ & $>99.99 \%$ \\
\hline Polarization sensitivity & $\begin{array}{l}\mathrm{MDP}<20 \%(99 \% \text { c.l. }) \text { for a } 10 \mathrm{mCrab} \text { source }\left(0.3-2 \mathrm{MeV}, T_{\mathrm{obs}}=1 \mathrm{yr}\right) \\
\text { Detection of a polarization fract. } \geq 20 \% \text { in more than } 20 \mathrm{GRBs} \text { per year }\end{array}$ \\
\hline$\Delta \mathrm{E} / \mathrm{E}$ (Gamma-ray imager) & $\begin{array}{l}3.0 \% \text { at } 1 \mathrm{MeV} \\
30 \% \text { at } 100 \mathrm{MeV}\end{array}$ \\
\hline$\Delta \mathrm{E} / \mathrm{E}$ (Calorimeter burst) & $\begin{array}{lr}<25 \% \text { FWHM at } & 0.3 \mathrm{MeV} \\
<10 \% \text { FWHM at } & 1 \mathrm{MeV} \\
<5 \% \text { FWHM at } & 10 \mathrm{MeV} \\
\end{array}$ \\
\hline Time tagging accuracy & 1 microsecond (at 3 sigma) \\
\hline $\begin{array}{l}\text { Impulsive event acquisition } \\
\text { logic (Calorimeter burst) }\end{array}$ & sub-millisecond trigger and photon-by-photon acquisition capability \\
\hline Orbit & $\begin{array}{l}\text { Low Earth Orbit, equatorial with inclination } i<2.5^{\circ} \text {, eccentricity } e<0.01 \text {, } \\
\text { altitude: } 550-600 \mathrm{~km}\end{array}$ \\
\hline $\begin{array}{l}\text { Average scientific } \\
\text { telemetry }\end{array}$ & $>1.4 \mathrm{Mbit} / \mathrm{s}$ (after data compression) \\
\hline $\begin{array}{l}\text { Satellite attitude } \\
\text { reconstruction }\end{array}$ & $1^{\prime}$ (at 3 sigma) \\
\hline Satellite pointing modes & $\begin{array}{l}\text { 1. pointing mode ( } 1 \text { or } 2 \text { pointings per orbit); } \\
\text { 2. survey zenith pointing mode. }\end{array}$ \\
\hline $\begin{array}{l}\text { Target of Opportunity } \\
\text { observations }\end{array}$ & within $6-12$ hours from alert (goal of $3-6$ hours) \\
\hline Mission duration & 3 years + provision for a $2+$ year extension \\
\hline
\end{tabular}

in a region of the electromagnetic spectrum, the socalled MeV domain, which is still largely unknown. The sensitivity requirement is relevant to all science drivers discussed above. Thus, the goal of detecting a significant number $(N>5)$ of SN Ia in gamma rays after 3 years requires a sensitivity in the $847 \mathrm{keV}$ line $<5 \times 10^{-6} \mathrm{ph} \mathrm{cm}^{-2} \mathrm{~s}^{-1}$ in $1 \mathrm{Ms}$ of integration time (Table $\mathrm{I}$ ).

- Another major requirement for a future gamma-ray observatory is to improve significantly the angular resolution over past and current missions, which have been severely affected by a spatial confusion issue. Thus, the e-ASTROGAM angular resolution will be excellent in the $\mathrm{MeV}$ range and above a few hundreds of $\mathrm{MeV}$, improving $C G R O / \mathrm{COMPTEL}$ and Fermi-LAT by almost a factor of 4 at $1 \mathrm{MeV}$ and $1 \mathrm{GeV}$, respectively. The targeted angular res-

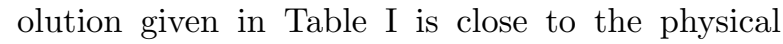
limits: for Compton scattering, the limit is given by the Doppler broadening induced by the velocity of the atomic electrons, while for low-energy pair production, the limit is provided by the nuclear recoil. e-ASTROGAM angular resolution will allow a number of currently unidentified gamma- 
ray sources (e.g. 992 sources in the 3FGL catalog 7]) to be associated with objects identified at other wavelengths. The GC region is the most challenging case, for which the e-ASTROGAM capability will be fully employed.

- The polarization sensitivity of e-ASTROGAM is designed to enable measurements of the gammaray polarization fraction in more than 20 GRBs per year (GRBs being promising candidates for highly gamma-ray polarized sources, see, e.g., 93.). Such measurements will provide important information on the magnetization and content (leptons, hadrons, Poynting flux) of the relativistic outflows, and, in the case of GRBs at cosmological distance, will address fundamental questions of physics related to vacuum birefringence and Lorentz invariance violation (e.g., 69]). With the designed polarization sensitivity, e-ASTROGAM will also be able to study the polarimetric properties of more than 50 pulsars, magnetars, and black hole systems in the Galaxy.

- The spectral resolution of e-ASTROGAM is well adapted to the main science drivers of the mission. Thus, the main gamma-ray lines produced in SN explosions or by LECR interactions in the ISM are significantly broadened by the Doppler effect, and a FWHM resolution of $3 \%$ at $1 \mathrm{MeV}$ is adequate. In the pair production domain, an energy resolution of $30 \%$ will be more than enough to measure accurately putative spectral breaks and cutoffs in various sources and distinguish the characteristic pion-decay bump from leptonic emissions.

- The timing performance of e-ASTROGAM is mainly driven by the physics of magnetars and rotation-powered pulsars, as well as by the properties of TGFs. The targeted microsecond timing accuracy is already achieved in, e.g., the AGILE mission [134.

The e-ASTROGAM requirements reflect the dual capacity of the instrument to detect both Compton scattering events in the 0.3 (and below) - $10 \mathrm{MeV}$ range and pair-producing events in the $10 \mathrm{MeV}-3 \mathrm{GeV}$ energy range; a small overlap around $10 \mathrm{MeV}$ allows (although in a limited energy band) cross-calibration, thus reducing systematic uncertainties. The main instrument features of e-ASTROGAM necessary to meet the scientific requirements in Table I] are described in Sect. IVB.

The sensitivity performance is consistent with the requirement of an equatorial low-Earth orbit (LEO) of altitude in the range $550-600 \mathrm{~km}$. Such an orbit is preferred for a variety of reasons. It has been demonstrated to be only marginally affected by the South Atlantic Anomaly and is therefore a low-particle background orbit, ideal for high-energy observations. The orbit is practically unaffected by precipitating particles originating from solar flares, a virtue for background rejection. Finally, both
ESA and ASI have satellite communication bases near the equator (Kourou and Malindi) that can be efficiently used as mission ground stations.

Table I also includes the most important system requirements such as the satellite attitude reconstruction, telemetry budget, and pointing capability. eASTROGAM is a multi-purpose astrophysics mission with the capability of a very flexible observation strategy. Two main scientific observation modes are to be managed by the Mission Operation Center (MOC):

- pointing mode;

- survey mode.

The pointing mode can be implemented either in a fixed inertial pointing or in the more efficient doublepointing per orbit mode. In the latter case, the eASTROGAM satellite is required to be able to perform two sky pointings per orbit, lasting approximately 40 minutes each. The survey mode consists in a continuous pointing to the zenith to perform a scan of the sky at each orbit. This mode can be activated at any time in principle, and depending on the scientific prioritization and on the mission schedule foreseen by the Science Management Plan, can lead to an optimized all-sky survey.

Requirements for the Ground Segment are standard for an observatory-class mission. Target of Opportunity observations (ToOs) are required to follow particularly important transient events that need a satellite repointing. The e-ASTROGAM mission requirement for ToO execution is within 6-12 hours, with the goal of reaching 3-6 hours. The speed of repointing depends on the torque of the reaction wheels. We expect a repointing velocity similar to Fermi ( 30 degrees/min, which grants to have a visible object in FoV within less than 5').

e-ASTROGAM does not use any consumable and could in principle be operated for a duration up to 10-20 years (well within the foreseen operation duration of 3 years with a possible extension of two), limited mainly by orbital instabilities and by the risk of accidents. Radiation damage in LEO, with almost equatorial inclination, is negligible. As an example, the degradation of Fermi, whose inclination implies significant crossing of the South Atlantic Anomaly, is negligible for what concerns electronics, negligible for what concerns Tracker aging, and around 1\%/year in terms of loss in light yield of the Calorimeter crystals.

Table II summarizes our conservative estimates of the number of sources detectable by e-ASTROGAM in 3 years, based on current knowledge and $\log N-\log S$ determinations of Galactic and extragalactic sources, including GRBs. It takes information from the the SwiftBAT 70-Month Hard X-ray survey catalog [26], the 4th INTEGRAL-IBIS catalog [32, and the 3rd Fermi-LAT catalog [7. Noteworthy, the latter catalog contains more than 1000 unidentified sources in the $100 \mathrm{MeV}-300$ $\mathrm{GeV}$ range with no counterparts at other wavelength, and most of them will be detected by e-ASTROGAM, in addition to a relevant number of new unidentified sources. 


\begin{tabular}{|l|l|l|}
\hline Type & $3 \mathrm{yr}$ & New sources \\
\hline Total & $3000-4000$ & $\sim 1800$ (including GRBs) \\
Galactic & $\sim 1000$ & $\sim 400$ \\
MeV blazars & $\sim 350$ & $\sim 350$ \\
GeV blazars & $1000-1500$ & $\sim 350$ \\
Other AGN $(<10 \mathrm{MeV})$ & $70-100$ & $35-50$ \\
Supernovae & $10-15$ & $10-15$ \\
Novae & $4-6$ & $4-6$ \\
GRBs & $\sim 600$ & $\sim 600$ \\
\hline
\end{tabular}

TABLE II. Estimated number of sources of various classes detectable by e-ASTROGAM in 3 years. The last column gives the number of sources not known before in any wavelength.

The discovery space of e-ASTROGAM for new sources and source classes is very large.

\section{THE SCIENTIFIC INSTRUMENT}

\section{A. Measurement principle and payload overview}

The e-ASTROGAM payload is shown in Figure 11. It consists of three main detectors:

- A silicon Tracker in which the cosmic gammarays undergo a first Compton scattering or a pair conversion; it is based on the technology of double sided Si strip detectors to measure the energy and the 3D position of each interaction with an excellent energy and spatial resolution;

- A 3D-imaging Calorimeter to absorb and measure the energy of the secondary particles; it is made of an array of small scintillation crystals $(33,856$ CsI (Tl) bars of $5 \times 5 \times 80 \mathrm{~mm}^{3}$ ) read out by silicon drift photodetectors to achieve the required energy resolution ( $4.5 \%$ at $662 \mathrm{keV})$;

- An Anticoincidence system (AC), composed of a standard AC shielding surrounding the top and four lateral sides of the instrument, and a Time-ofFlight unit located below the instrument, to veto the particle background arising from the platform; it is made of plastic scintillator tiles with a detection efficiency exceeding 99.99\%.

The payload is completed by a Payload Data Handling Unit (PDHU) and a Power Supply Unit (PSU) located below the Calorimeter inside the platform together with the back-end electronics (BEE). The PDHU is in charge of the payload internal control, the scientific data processing, the operative mode management, the on-board time management, and the telemetry and telecommand management. The total payload mass and power budget (including maturity margins) are $999 \mathrm{~kg}$ and $1340 \mathrm{~W}$, respectively.

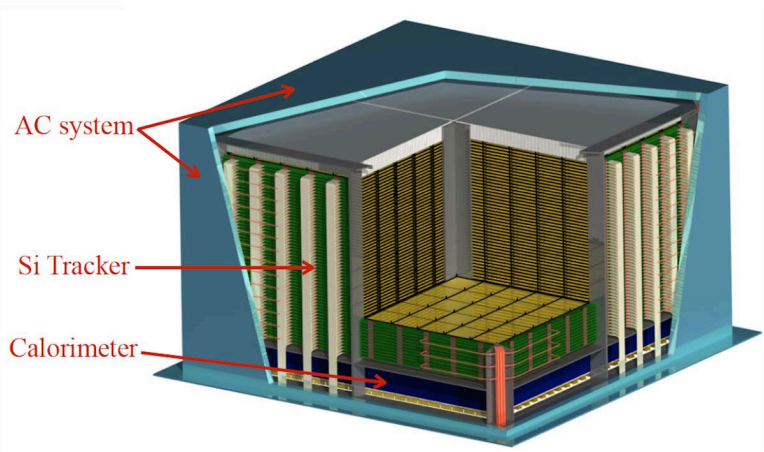

FIG. 11. Overview of the e-ASTROGAM payload showing the silicon Tracker, the Calorimeter and the Anticoincidence system.

Interactions of photons with matter in the eASTROGAM energy range is dominated by Compton scattering from (below) $0.2 \mathrm{MeV}$ up to about $15 \mathrm{MeV}$ in silicon, and by $e^{+} e^{-}$pair production in the field of a target nucleus at higher energies. e-ASTROGAM maximizes its efficiency for imaging and spectroscopy of energetic gamma-rays by using both processes. Figure 12 shows a schematic representation of topologies for Compton and pair events.

For pair-production events, e-ASTROGAM is similar in design to AGILE and Fermi-LAT, but optimized for lower energy. This goal is achieved by eliminating the passive converters used in both these instruments. This approach reduces gamma-ray conversion efficiency, but it improves the instrument point-spread function (PSF) by reducing absorption and multiple Coulomb scattering of the electron and positron. The broad PSF is a primary limiting factor in the science that can be done at energies below $100 \mathrm{MeV}$ by AGILE and Fermi-LAT. Pair events produce two main tracks from the created electron and positron. Tracking of the initial opening angle and of the plane spanned by the electron and positron tracks enables direct back-projection of the source position. Multiple scattering of the pair in the tracker material leads to broadening of the tracks and limits the angular resolution. The nuclear recoil taking up an unmeasured momentum results in an additional small uncertainty. The energy of the gamma-ray is measured using the Calorimeter and information on the electron and positron multiple scattering in the Tracker. Polarization information in the pair domain is given by the azimuthal orientation of the electron-positron plane; in addition to improving the PSF, the use of low-mass tracker planes also enables photon polarization measurements.

Detecting gamma-rays by Compton scattering is more complicated than for pair production, because the scattered photon carries a significant amount of the information about the incident photon and thus it needs to be detected too. In practice, a Compton telescope requires two separate photon interactions in order to have a clear detection. The first Compton scattering of the incident 


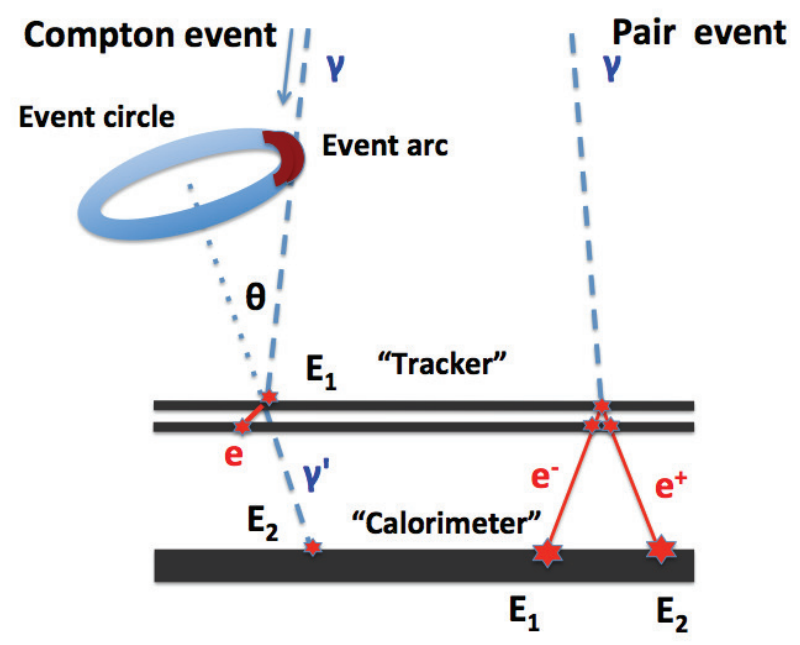

FIG. 12. Representative topologies for a Compton event (left) and for a pair event (right). Photon tracks are shown in pale blue, dashed, and electron and/or positron tracks in red, solid. From 96.

photon occurs in one of the Tracker planes, creating an electron and a scattered photon. The Tracker measures the interaction location, the electron energy, and in some cases the electron direction. The scattered photon can be absorbed in the Calorimeter or (with smaller probability) scattered a second time in the Tracker before being absorbed in the Calorimeter where its energy and absorption position are measured.

The basic principle of the Compton mode of operation is illustrated in Figure 12 left. An incident gammaray Compton scatters by an angle $\Theta$ in one layer of the Tracker, transferring energy $E_{1}$ to an electron. The scattered photon is then absorbed in the Calorimeter, depositing an energy $E_{2}$, and the scattering angle is given by $\cos \Theta=m_{e} c^{2} / E_{2}+m_{e} c^{2} /\left(E_{1}+E_{2}\right)$, where $m_{e}$ is the electron mass. With this information, one can derive an "event circle" from which the original photon arrived. We will call "untracked" this sort of Compton events. The uncertainty in the event circle reconstruction is reflected in its width and is due to the uncertainties in direction reconstruction of the scattered photon and the energy measurements of the scattered electron $\left(E_{1}\right)$ and the scattered photon $\left(E_{2}\right)$. Multiple photons from the same source enable a full deconvolution of the image, using probabilistic techniques.

For energetic Compton scatters (above $\sim 1 \mathrm{MeV}$ ), measurement of the track of the scattered electron becomes possible, resulting in a reduction of the event ring to an arc, hence further improving event reconstruction. If the scattered electron direction is measured, the event circle reduces to an event arc with length due to the uncertainty in the electron direction reconstruction, allowing improved source localization. This event is called "tracked", and its direction reconstruction is somewhat similar to that for pair event - the primary photon direction is reconstructed from the direction and energy of two secondary particles: scattered electron and photon. Redundant kinematic information from multiple interactions could also help. Compton scattering angles depend on polarization of the incoming photon, hence careful statistical analysis of the photons for a strong (e.g., transient) source yields a measurement of the degree of polarization of its high-energy emission (e.g. [58]).

Especially for the Compton mode at low energies, but also more broadly over the entire energy range covered by e-ASTROGAM, it is important to keep the amount of passive materials on the top and at the sides of the detector to a minimum, to reduce background in the field of view and to optimize angular and energy resolutions. In addition, the passive materials between the Tracker layers, and between the Tracker and the Calorimeter, must be minimized for best performance.

\section{Silicon Tracker}

The Si Tracker is the heart of the e-ASTROGAM payload. It is based on the silicon strip detector technology widely employed in medical imaging and particle physics experiments (e.g. ATLAS and CMS at LHC), and already applied to the detection of gamma-rays in space with the AGILE and Fermi missions. The eASTROGAM Tracker needs double sided strip detectors (DSSDs) to work also as a Compton telescope.

The essential characteristics of the e-ASTROGAM Tracker are:

- its light mechanical structure minimizing the amount of passive material within the detection volume to enable the tracking of low-energy Compton electrons and $e^{+} e^{-}$pairs, and improve the point spread function in both the Compton and pair domains by reducing the effect of multiple Coulomb scattering;

- its fine spatial resolution of less than $40 \mu \mathrm{m}(<1 / 6$ of the microstrip pitch) obtained by analog readout of the signals (as in the AGILE Tracker);

- its charge readout with a very good spectral resolution of $\sim 6 \mathrm{keV}$ FWHM (noise level in the baseline configuration; the statistical contribution to the energy resolution is negligible in the relevant range of deposited energy $(E<500 \mathrm{keV})$, so the energy resolution is practically independent of energy and corresponds to the noise level) obtained with an ultra low-noise FEE, in order to accurately measure low-energy deposits produced by Compton events; the energy threshold is $15 \mathrm{keV}$.

The Si Tracker comprises 5600 DSSDs arranged in 56 layers (100 DSSDs per layer). It is divided in four towers of $5 \times 5$ DSSDs. The spacing of the Si layers is of $10 \mathrm{~mm}$. 
The total detection area amounts to $9025 \mathrm{~cm}^{2}$ and the total Si thickness to $2.8 \mathrm{~cm}$, which corresponds to $0.3 \mathrm{ra}-$ diation length on axis, and a probability of a Compton interaction at $1 \mathrm{MeV}$ of $40 \%$. Such a stacking of relatively thin detectors enables an efficient tracking of the electrons and positrons produced by pair conversion, and of the recoil electrons produced by Compton scattering. The DSSD signals are read out by 860160 independent, low-power, electronics channels with self-triggering capability.

a. Silicon detectors The active element is a Si DSSD of $500 \mu \mathrm{m}$ thickness and $9.5 \times 9.5 \mathrm{~cm}^{2}$ area, with electrodes of $100 \mu \mathrm{m}$ width, and $240 \mu \mathrm{m}$ pitch (corresponding to 384 microstrips per side), a guard ring of $1.5 \mathrm{~mm}$, and polysilicon resistors for the bias. It can be manufactured from high resistivity $(R \geq 5 \mathrm{k} \Omega \mathrm{cm}) 6$ " substrate by, e.g., the Silicon Radiation Sensors $\left.{ }^{(}\right)$group of the Fondazione Bruno Kessler FBK (SRS-FBK) or Hamamatsu Photonics ${ }^{\circledR}$.

Each layer of a tower contains $5 \times 5$ DSSDs, which are chained together with wire bonding strip to strip. Ladders of five Si tiles are first assembled and then bonded to five other ladders in the orthogonal direction. Si strip bonding is now a standard technology previously used in, e.g., the Fermi/LAT and AGILE Tracker and the PAMELA and AMS-02 cosmic-ray experiments.

b. Mechanical structure The mechanical structure holding a tray of $5 \times 5$ DSSDs with the associated FEE is composed of two frames sandwiching the Si detectors, the rods direction of the upper frame being orthogonal to that of the lower frame, to form a grid. On each side, the support rods are parallel to the DSSD strips to enable the wire bonding. The DSSDs are glued onto the frames with a structural adhesive, and a Kapton ${ }^{(C)}$ foil is added in the middle of the glue thickness to ensure electrical insulation. The frames, which are $2 \mathrm{~mm}$ thick, are made of a polymeric resin reinforced by high modulus carbon fibers woven into fabrics. Carbon fiber spacers determine a total spacing of the Si layers of $10 \mathrm{~mm}$; placed at the crossing between frames, they limit the vertical displacements under loads achieving a uniform distribution of the displacement among them and realize unilateral connections between the trays. The towers of the tracker are assembled together by a structural mainframe composed of vertical fixing aluminum columns. In order to increase the stiffness of each tower, the baseline configuration of the Tracker comprises two honeycomb panels (e.g. Hexel $3 / 16-5052+1 \mathrm{~mm}$ thick carbon fiber foils), one at the top ( $4 \mathrm{~cm}$ thickness) and one at the bottom $(1 \mathrm{~cm}$ thickness).

The Tracker mechanical design is the result of detailed structural calculation based on a simplified Finite Element Model (FEM) and including both static and modal analyses. The maximum vertical displacement obtained by FEM is of 280 microns (Figure 13), far below the rupture limit of Si detectors.

c. Front-End Electronics The FEE is distributed over the four sides of the Tracker (there are no electron- ics between the four towers), the detector microstrips being connected to the FEE ASICs through pitch adapters. The FEE ASICs are the 32-channels IDeF-X HD circuits developed at CEA/Saclay. The 860,160 DSSD signals are read by a total of 26,880 ASICs $(4 \times 120$ ASICs per layer $)$.

IDeF-X HD is a $5.8 \times 2.5 \mathrm{~mm}^{2}$ chip operated at $3.3 \mathrm{~V}$ and consuming $800 \mu \mathrm{W} /$ channel (static dissipated power). It was built based on the AMS CMOS $0.35 \mu \mathrm{m}$ technology using the full custom Rad-Hard libraries, and combine the most recent developments for space applications, including fully space-qualified devices 64, 90. In particular, IDeF-X HD was extensively studied with respect to radiation tolerance (Single Event Latchup (SEL) - free; Single Event Effect $(\mathrm{SEE})>9 \mathrm{MeV} \mathrm{cm}^{2} \mathrm{mg}^{-1}$; Total Ionizing Dose (TID) $>300 \mathrm{krad} \mathrm{w} / \mathrm{o}$ effect on noise response), as it was selected for the FEE of the STIX instrument of the Solar Orbiter mission.

Each individual channel of IDeF-X HD is made of: a charge sensitive preamplifier (CSA) optimized for low current $(<1 \mathrm{nA})$ and capacitance around $10 \mathrm{pF}$, a variable gain (inverting or non inverting) stage, a pole-zero cancellation stage, an adjustable shaper in the range from 1 to $11 \mu$ s (peaking time), a baseline holder, providing a stable offset whatever the leakage current into a channel, a peak detector and hold, and a discriminator (each channel, 6 bit DAC) in the range from 0 to $13 \mathrm{keV}$ for $\mathrm{Si}$ (the settings is not linear to allow fine tuning in the low range and coarse in the high range). Together with the 32 inputs (DC or AC coupled), the other interfaces of IDeF-X HD are two differential analog output and two independent slow controls. The CSA includes a continuous reset feedback circuitry, which includes a non stationary noise suppressor to optimize the noise response into the whole dynamic range. The dynamic range is $10 \mathrm{fC}$ (but is programmable up to $40 \mathrm{fC}$ ).

The analog output signals of IDeF-X will be converted to digital signals with the OWB-1 ADC integrated system. OWB-1 is a low noise (0.6 LSB), low power $(1 \mathrm{~mW}$ per active channel) chip of $11.55 \mathrm{~mm}^{2}$ area, including parallel Wilkinson ADC for 16 differential channels with a real 13 bits resolution at a conversion rate of $2.8 \mu \mathrm{s}$ (and 11 bits resolution at $0.9 \mu$ s conversion rate). The chip was also built from the AMS CMOS $0.35 \mu \mathrm{m}$ technology and it is radiation hard by design (SEL hardened + Single Event Upsets flag). Qualification procedure of this ASIC is on going.

\section{Calorimeter}

The e-ASTROGAM Calorimeter is a pixelated detector made of a high- $Z$ scintillation material - Thallium activated Cesium Iodide - for an efficient absorption of Compton scattered gamma-rays and electron-positron pairs. It consists of an array of 33,856 parallelepiped bars of $\mathrm{CsI}(\mathrm{Tl})$ of $8 \mathrm{~cm}$ length and $5 \times 5 \mathrm{~mm}^{2}$ cross section, read out by silicon drift detectors (SDDs) at both ends [63], arranged in an array of $529(=23 \times 23)$ elemen- 

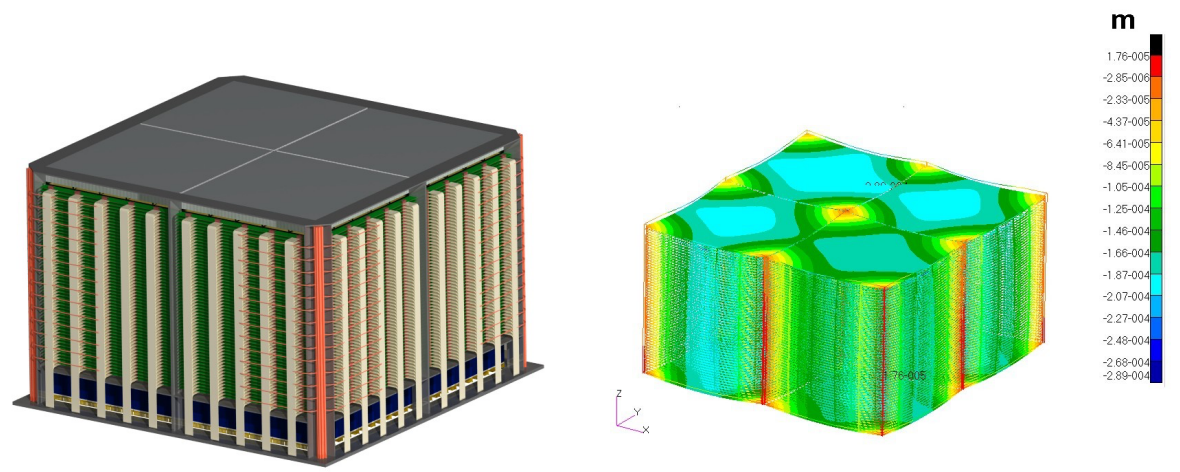

FIG. 13. Left panel - Overview of the Tracker mechanical design. Right panel - Illustration of the Tracker structural analysis showing normalized deformation of elements.

tary modules comprising each 64 crystals (see Figure 14). The Calorimeter thickness $-8 \mathrm{~cm}$ of $\mathrm{CsI}(\mathrm{Tl})$ - makes it a 4.3 radiation-length detector having an absorption probability of a $1-\mathrm{MeV}$ photon on axis of $88 \%$.

The Calorimeter detection principle and architecture are based on the heritage of the space instruments $I N$ TEGRAL/PICsIT, AGILE/MCAL and Fermi/LAT, as well as on the particle physics experiment LHC/ALICE at CERN. However, the e-ASTROGAM calorimeter features two major improvements with respect to the previous instruments:

- the energy resolution is optimized to a FWHM of $4.5 \%$ at $662 \mathrm{keV}$ (scaling with the inverse of the square root of the energy) by the use of low-noise SDDs for the readout of the scintillation signals, combined with an appropriate ultra low-noise FEE;

- the spatial resolution is improved by measuring the depth of interaction in the detector from a suitable weighting function of the recorded scintillation signals at both ends; the position resolution along the $\mathrm{CsI}(\mathrm{Tl})$ bars is $\sim 5 \mathrm{~mm} \mathrm{FWHM}$, i.e. comparable to the resolution in the $\mathrm{X}-\mathrm{Y}$ plane given by the crystal cross section $\left(5 \times 5 \mathrm{~mm}^{2}\right)$. Accurately measuring the 3D position and deposited energy of each interaction is essential for a proper reconstruction of the Compton events.

The simultaneous data set provided by the Silicon Tracker, the Calorimeter and the Anticoincidence system constitutes the basis for the gamma-ray detection. However, thanks to the detector excellent granularity, Calorimeter-only events can be used on board to provide a burst notice and a first approximate localization via fast onboard reconstruction even in the absence of a signal from the Tracker. This data acquisition mode is dedicated to the search for fast transient events such as GRBs and TGFs. The corresponding trigger condition is explained in Sect. IV A 5 .

a. Scintillation crystals and housing The choice of $\mathrm{CsI}(\mathrm{Tl})$ as the scintillation material in the baseline design

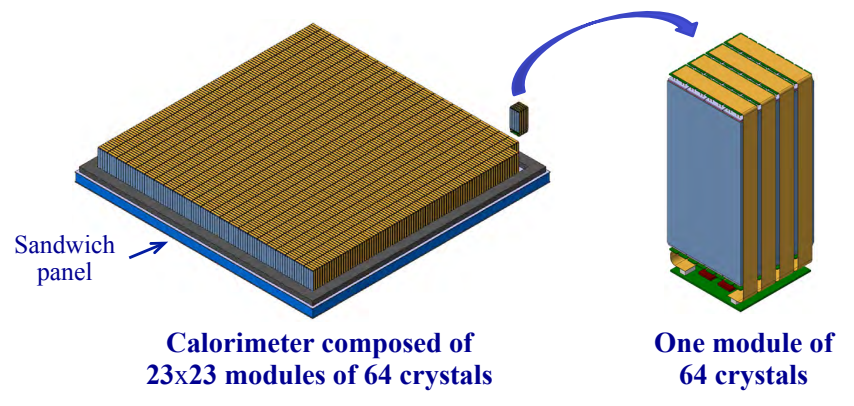

FIG. 14. Overview of the Calorimeter and of one of its 529 $(=23 \times 23)$ basic modules comprising $64 \mathrm{CsI}(\mathrm{Tl})$ crystals.

is motivated by the facts that (i) it is one of the brightest scintillator (light yield of 54 photons per keV), (ii) it matches well to the response of Si photodiodes (broad emission spectrum with a maximum at $540 \mathrm{~nm}$ ), (iii) it is only slightly hygroscopic (much less than, e.g., $\mathrm{LaBr}_{3}$ and $\mathrm{CeBr}_{3}$ ), (iv) it has good radiation hardness properties, and (v) it has already flown on, e.g., the INTEGRAL, AGILE and Fermi satellites. The required 33,856 CsI(Tl) bars could be produced by, e.g., Saint Gobain Crystals ${ }^{\circledR}$ or Detec Europe ${ }^{\circledR}$.

The possibility of using a new scintillator technology that could offer an improved energy resolution and a reduced scintillation decay time will be studied during the assessment phase. Several potential candidates exist, including RGB $\left(\mathrm{RbGd}_{2} \mathrm{Br}_{7}\right.$ :Ce) and lutetium-based scintillators. New developments in nano-crystalline glass ceramic scintillators also offer sufficient promise as to be worthy of further investigation. There is a very active development ongoing within the e-ASTROGAM consortium on this topic, with ESA support, and the results are encouraging.

Each crystal will be wrapped with a reflective material in order to optimize the light collection and reduce the optical cross talk. The selected reflective material is the Radiant Mirror Film ESR from 3M, with a thickness of 
$63 \mu \mathrm{m}$. This wrapping material, which is a non-metallic multilayer polymer, was previously employed for the CsI crystals of the Fermi/LAT calorimeter, as well as, e.g., for the calorimeters of the JLAB/DVCS and GSI/PANDA particle physics experiments.

b. Photosensors: silicon drift detectors Silicon Drift Detectors (SDDs) are solid-state devices suitable for both direct X-ray and scintillation light detection 63. Their peculiar characteristic is the fact that the signal charge is driven toward a small collecting anode by means of a suitably tailored electric field within the depletion region. Under these conditions, a very small output capacitance can be obtained, almost independent from the active area, and hence an improvement of more than an order of magnitude in noise performance with respect to PIN photodiode of equivalent active area. When SDDs are used as readout devices for $\mathrm{CsI}(\mathrm{Tl})$ scintillation crystals, the improved noise performance results in a very good energy resolution (4.5\% FWHM at $662 \mathrm{keV})$ and an energy threshold lower than $30 \mathrm{keV}$ [91. For the e-ASTROGAM calorimeter we foresee to use SDDs designed and developed at INFN Trieste, and fabricated at the FBK (SRS-FBK). A 6" wafer processing line was successfully set up at SRS-FBK, with mass production capabilities.

In the e-ASTROGAM calorimeter, the basic detector element is formed by the coupling of four $\mathrm{CsI}(\mathrm{Tl})$ bars to two square arrays of $2 \times 2 \mathrm{SDDs}$ of $5 \mathrm{~mm}$ side. The $\mathrm{CsI}(\mathrm{Tl})$ scintillator bars will be optically coupled to two SDD arrays, one on top and the other at the bottom of the crystals, to enable the reconstruction of the energy and position of interaction along the bars by means of a suitable weighting function of the recorded signals at both ends. This hodoscopic architecture was already employed for the AGILE and Fermi LAT calorimeters, but using much larger crystals and PIN photodiodes as readout devices. The improved noise performance of the SDDs will allow us to obtain a depth-of-interaction resolution of about $5 \mathrm{~mm}$ FWHM when coupled to $80 \mathrm{~mm}$ long scintillator bars, as already demonstrated at laboratory level [88].

c. Mechanical structure The e-ASTROGAM Calorimeter is made of $529(=23 \times 23)$ elementary modules, each of which comprising 16 basic elements of four $\mathrm{CsI}(\mathrm{Tl})$ bars coupled to two square arrays of $2 \times 2$ SDDs. This gives a total of 33,856 identical crystals and 67,712 SDDs. The surface covered by the calorimeter is about $100 \times 100 \mathrm{~cm}^{2}$. The spacing between the Calorimeter and the Tracker (i.e. the distance between the Calorimeter and the bottom honeycomb panel of the Tracker structure) is $2 \mathrm{~mm}$.

An exploded view of a basic module of the Calorimeter is shown in Figure 15. The crystals are supported by an alveolar structure made of a polymer resin reinforced by high modulus carbon fibers, with four crystals (i.e. one detector element) contained in each cell. The thickness of the structure walls is $200 \mu \mathrm{m}$. Each alveolus is epoxy-glued to an insert in carbon fibers, which makes the interface with the support elements at the bottom of the module. Each module is fixed to a bottom sandwich panel composed of a $3 \mathrm{~cm}$ honeycomb core covered by carbon fiber skins. The sandwich panel is stiff enough to hold the weight of the 33,856 crystals of the calorimeter $(305 \mathrm{~kg})$. At the top of the alveolar structure, a carbon plate is added to further limit the displacement of the crystals.

The FEE boards with the OWB-1 ADC ASICs (see below) are positioned under the crystals on dedicated supporting circuits. The SDD boards with the VEGA analog ASICs in contact of the collecting anodes are connected to the ADC ASIC boards via Kapton foils (Figure 15. The low heat generated by the SDD boards $(2 \times 27 \mathrm{~mW}$ per module) is also evacuated by the Kapton circuits.

d. Front-End Electronics We foresee using for the FEE ASICs a modified version of the VEGA circuits developed by Politecnico di Milano and Università di Pavia. With the development of a new chip of 4 channels, a total of 16,928 ASICs will be needed to read the 67,712 SSD signals.

The VEGA ASIC 18, which was specifically developed for SDD readout, is realized in CMOS AMS 0.35 $\mu \mathrm{m}$ technology. It is a low power circuit, consuming $420 \mu \mathrm{W} /$ channel, which contains a preamplifier, a programmable shaper-amplifier, a discriminator and a peak stretcher. It provides an Equivalent Noise Charge (ENC) of 16 electrons rms at $3 \mu$ s shaping time when coupled to a $350 \mathrm{fF}$ input capacitance SDD. With this system, an energy resolution of $183 \mathrm{eV}$ FWHM at $5.9 \mathrm{keV}$ has been obtained. The ASIC includes a linear array of 32 independent channels with dimensions of $200 \times 500 \mu \mathrm{m}$ per channel, each with a dynamic range of $60 \mathrm{keV}$ for X-ray direct detection in Si (equivalent to about 16,400 electrons).

A minimal set of optimization steps will be carried out to tailor the characteristics of the VEGA ASIC to the e-ASTROGAM calorimeter requirements. In particular, considering a typical photoelectron yield of about 30 electron per $\mathrm{keV}$ obtainable for $\mathrm{CsI}(\mathrm{Tl})$ scintillators coupled to silicon devices, the shaping-amplifier dynamic range should be increased by a factor of $\sim 20$ (equivalent to $\sim 10 \mathrm{MeV}$ detection in $\mathrm{CsI}(\mathrm{Tl}))$.

The analog output signals of the new VEGA ASICs will be converted to digital signals with the same OWB1 ADC integrated system as used for the Tracker FEE. The $2 \times 64$ signals of a module will be processed by four 32-channels OBW-1 ASICs placed on a dedicated board below the crystals (Figure 15.

\section{Anticoincidence System}

The third main detector of the e-ASTROGAM payload consists of an Anticoincidence system composed of two main parts: (1) a standard Anticoincidence, named Upper-AC, made of segmented panels of plastic scintillators covering the top and four lateral sides of the instru- 


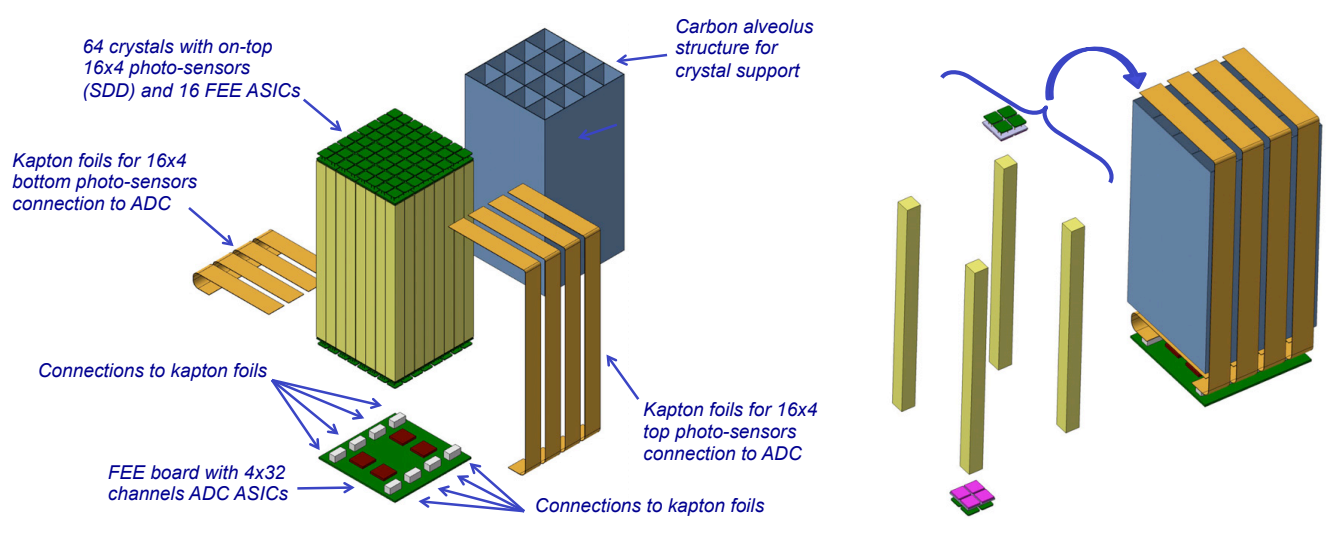

FIG. 15. Exploded view drawing of a elementary module of the Calorimeter with its alveolus structure.

ment, requiring a total active area of about $5.2 \mathrm{~m}^{2}$, and (2) a Time of Flight (ToF), aimed at rejecting the particle background produced by the platform. The Upper$\mathrm{AC}$ detector is segmented in 33 plastic tiles (6 tiles per lateral side and 9 tiles for the top). All scintillator tiles are coupled to silicon photomultipliers (SiPM) by optical fibers. The architecture of the Upper-AC detector is fully derived from the successful design of the AGILE 108 and Fermi/LAT 95 AC systems. In particular, their segmentation has proven successful at limiting the "backsplash" self-veto, therefore the dead time of the instrument. The Upper-AC particle background rejection is designed to achieve a relativistic charged particle detection inefficiency lower than $10^{-4}$, a standard value already realized in current space experiments. In addition to the panel segmentation, providing coarse information on the part of the detector that has been hit, we are also considering the possibility of even finer position resolution based on the analysis of the relative light output of multiple fibers.

In the baseline design, the Upper-AC system covers the entire instrument from five sides, leaving open the bottom for design considerations of cabling to the S/C bus, the layout of heat pipes, etc. The bottom side of the instrument is protected by the ToF to discriminate the particles coming out from the instruments from those entering the instrument from below. The ToF is composed by two scintillator layers separated by $50 \mathrm{~cm}$. The required timing resolution is of $300 \mathrm{ps}$. The readout will be performed by SiPM connected with Time Digital Converter (TDC). The ToF will be based on technologies well proven in space (AMS and PAMELA satellites).

The plastic scintillator type that was selected for both Upper-AC and ToF is the BC400 or BC408 from Saint Gobain Crystals ${ }^{\circledR}$ or the equivalent EJ212 or EJ200 from Eljen Technology ${ }^{\circledR}$. For all these materials, the scintillator peak emission is around $425 \mathrm{~nm}$. The scintillator thickness has been set to a minimum of $5 \mathrm{~mm}$ for the Upper-AC lateral panels and $6 \mathrm{~mm}$ for the Upper-AC top panel and ToF in order to get enough light to detect more than $99.99 \%$ of the passing through relativistic charged particles. The energy threshold of the AC detectors is set to $100 \mathrm{keV}$. The light emitted by the plastic is transferred to the SiPM through wavelength shifting optical fibers. The combination of optical fibers and SiPM provides the best solution to collect the scintillator optical light, as it has the higher gain and gives the best efficiency of charged particle rejection. SiPM has also the advantage over traditional photomultiplier tubes to work at low bias (few tens of volts), so without the need of high voltage. The selected SiPM could be from the BSeries (blue sensitive) of the SensL ${ }^{\circledR}$ company. The peak sensitivity of these photosensors is at $420 \mathrm{~nm}$ (within a sensitivity range of $300-800 \mathrm{~nm}$ ), which copes well with the plastic scintillator emission. SensL ${ }^{\circledR}$ SiPMs were recently studied with respect to radiation tolerance in an ESA-led program and are now at technology readiness level (TRL) 5. For the Upper-AC readout we foresee the selection of the VATA64 ASIC from the Ideas Company, this chip being optimized for SiPM readout and already space qualified (see Ref. 24]). Alternative options will be considered for the ToF, such as the MUSIC ASIC, which has also been developed for SiPM readout, and has a Single Photon Resolution Time of about 100 ps rms [67].

\section{Data Handling and Power Supply}

The e-ASTROGAM payload is completed by a Payload Data Handling Unit (PDHU) and a Power Supply Unit (PSU). The PDHU is in charge of carrying out the following principal tasks: (i) payload internal control; (ii) scientific data processing; (iii) operative modes management; (iv) on board time management; (v) Telemetry and Telecommand management. The main functions related to the scientific data processing are: (i) BEE interfacing through dedicated links to acquire the scientific data; (ii) the real-time software processing of the collected silicon Tracker, Anticoincidence and Calorimeter scientific data aimed at rejecting background events to meet the telemetry requirements; (iii) scientific data compression; (iv) formatting of the compressed data into telemetry 


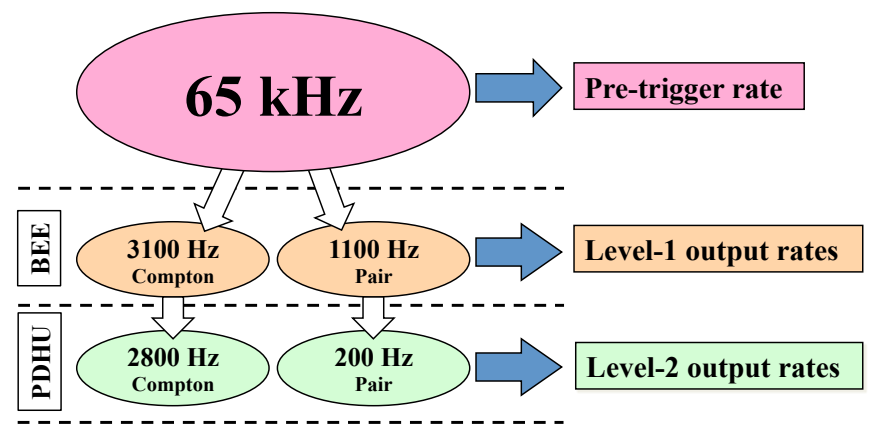

FIG. 16. Expected data flow of the on-board e-ASTROGAM gamma-ray data acquisition system.

packets.

The heart of the PDHU architecture is based on a powerful Digital Signal Processor (DSP) running the payload on-board software. Considering the large amount of events to be processed, a possible DSP could be the HiRel component C6727B-250 produced by Texas Instruments ${ }^{\circledR}$. This floating point $\mathrm{CPU}$ running at 250 $\mathrm{MHz}$ is capable of 500 MMACS (2000 MIPS / 1500 MFLOPS) by executing up to 8 instructions in parallel (6 of which floating points).

The PSU is in charge of generating the required payload voltages with high DC/DC conversion efficiency and distributing them to the other sub-systems.

\section{Trigger logic and data flow architecture}

The e-ASTROGAM on-board scientific data processing is composed of two main trigger pipelines, the gamma-ray acquisition mode and the Calorimeter burst search. Both are based on the experience of the AGILE and Fermi missions. The simultaneous data sets provided by the silicon Tracker, the Calorimeter and the AC constitute the basis for the gamma-ray detection and processing. The gamma-rays trigger logic is structured on two main levels: Level-1 (fast: 5-10 $\mu$ s logic, hardware); and Level-2 (asynchronous, $50 \mu$ s processing, software). Figure 16 shows the expected data rates at the input of the Level- 1 and at the output of the Level- 1 and Level-2 trigger stages.

Level-1 is a hardware trigger logic with fast response implemented in the silicon Tracker BEE providing a preliminary discrimination between Compton and pairproducing photon events and a first cut of background events. Discrimination criteria based on the hit multiplicity in the Tracker and in the Calorimeter can provide optimal algorithms to identify Compton events. The Level-1 trigger configuration is defined to save the largest possible number of potential Compton events.

Level-2 is a software trigger stage carried out by the PDHU and aimed, at further reducing the residual particle and photon background of the pair data set and at finalizing the selection of the Compton events. The heart of the Level-2 trigger stage consists of the track reconstruction of the candidate pair events implemented with Kalman Filter techniques. The Level-2 trigger is a full asynchronous processing stage and does not increase the dead time of the instrument. At the end, the Compton events and the pair events surviving the Level-2 trigger are collected in dedicated telemetry packets and sent to the ground.

Level-1 is has a rate tuned to limit the dead time and to fit the accepted event rate into the acceptance capability of the onboard reconstruction stage. Level-2, which is fed by events passing Level-1, is tuned so that the rate of accepted events fits into telemetry bandwidth. Only events passing Level-2 are downlinked.

The Calorimeter burst search is a software algorithm implemented by the PDHU. The burst search is based on the integration and processing of a proper set of rate meters measuring the trend of the background and foreground counting rates. Since the expected impulsive signals (GRBs and TGFs) are strongly energy- and timescale-dependent, the rate meters are integrated on a wide range of timescales $(0.1 \mathrm{~ms}-10 \mathrm{~s})$ and energy ranges (in the overall range $30 \mathrm{keV}-200 \mathrm{MeV}$ ). Assuming a typical GRB spectrum, the energy channels are defined in order to obtain a homogeneous threshold over the whole energy range.

The burst search logic routinely compares the background and foreground rate meters. Elementary triggers are generated when foreground rate meters show an overthreshold counting excess respect to the background estimation. Finally, the triggers have to be validated by satisfying coincidence conditions on the different energy channels aimed at spurious trigger rejection. These conditions are implemented using look-up tables fully programmable from ground.

A cyclic buffer is required to routinely save the events; the size of this buffer is defined in order to store $100 \mathrm{~s}$ of background. Following a burst trigger, the logic will be able to identify the beginning and the end of the segment of data acquisition and then transfer it to ground by telemetry.

\section{B. Performance assessment}

The scientific performance of the e-ASTROGAM instrument was evaluated by detailed numerical simulations with the software tools MEGAlib and BoGEMMS. The MEGAlib package [153] was originally developed for analysis of simulation and calibration data related to the Compton scattering and pair-creation telescope MEGA [81. It has then been successfully applied to a wide variety of hard X-ray and gamma-ray telescopes on ground and in space, such as COMPTEL, NCT, and NuSTAR. BoGEMMS (Bologna Geant4 Multi-Mission Simulator) is a software for simulation of payload of Xand gamma-ray missions, which has been developed at the INAF/IASF Bologna [40. It has already been ap- 


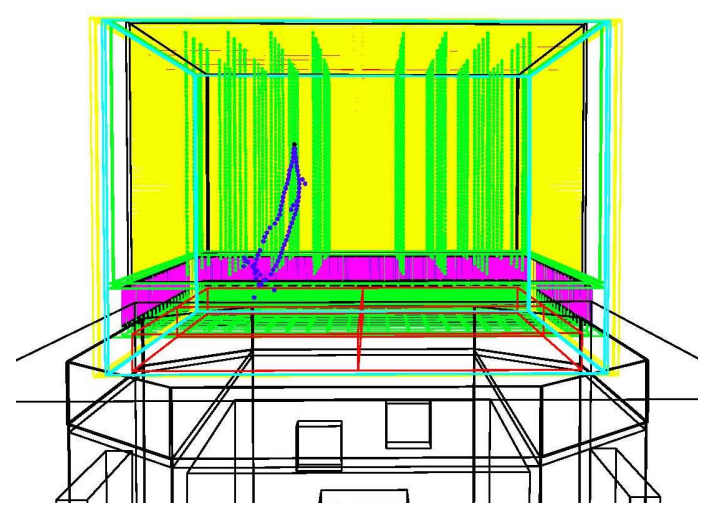

FIG. 17. Geant4/MEGAlib mass model of the eASTROGAM telescope, with a simulated pair event (in blue) produced by a $30-\mathrm{MeV}$ photon. The vertical green lines represent the carbon fiber spacers placed between the DSSD layers to increase the stiffness of the Tracker (see Sect. IV A 1.

plied to several hard X-ray/gamma-ray instruments and mission projects, including Simbol-X, NHXM, GammaLight, AGILE, and GAMMA-400. Both software packages exploit the Geant4 toolkit to model the geometrical and physical parameters of the detectors and simulate the interactions of photons and particles in the instrument.

The numerical mass model of e-ASTROGAM used to simulate the performance of the instrument is shown in Figure 17. An accurate mass model that includes passive material in the detector and its surroundings, true energy thresholds and energy and position measurement accuracy, as well as a roughly accurate S/C bus mass and position are crucial to the modeling. In particular, care was taken to include all passive materials close to the $\mathrm{Si}$ and $\mathrm{CsI}(\mathrm{Tl})$ detectors.

\section{Background model}

For best environmental conditions, e-ASTROGAM should be launched into a quasi-equatorial (inclination $\left.i<2.5^{\circ}\right)$ LEO at a typical altitude of $550 \mathrm{~km}$. The background environment in such an orbit is now well-known (Figure 18), thanks to the Beppo-SAX mission, which measured the radiation environment on a low-inclination $\left(i \sim 4^{\circ}\right), 500-600 \mathrm{~km}$ altitude orbit almost uninterruptedly during 1996 - 2002 [41] and the on-going $A G$ ILE mission, which scans the gamma-ray sky since 2007 from a quasi-equatorial orbit at an average altitude of $535 \mathrm{~km}$ [134. The dominant sources of background for the e-ASTROGAM telescope in the $\mathrm{MeV}$ domain are the cosmic diffuse gamma-ray background, the atmospheric gamma-ray emission, the reactions induced by albedo neutrons, and the background produced by the radioactivity of the satellite materials activated by fast protons and alpha particles. All these components were modeled in detail using the MEGAlib environment tools. In the pair domain above $10 \mathrm{MeV}$, the background is mainly

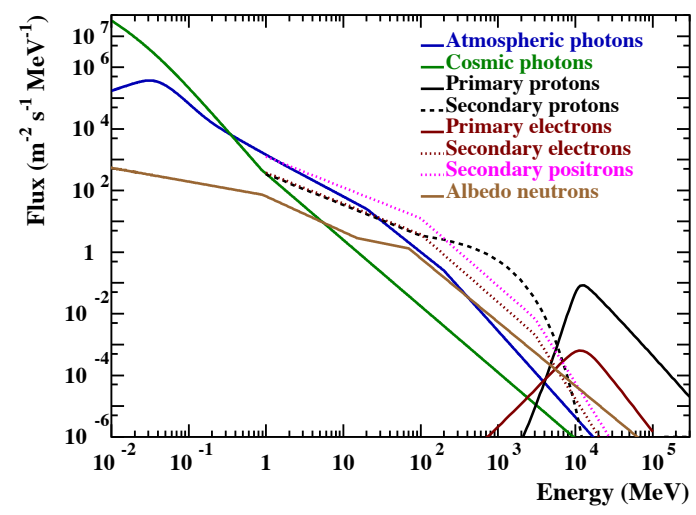

FIG. 18. Background environment of e-ASTROGAM on its orbit. The satellite will be exposed to Galactic CR (mainly protons and electrons) modulated by the geomagnetic field, semi-trapped secondary protons and leptons, as well as to albedo neutrons and atmospheric gamma rays. The cosmic diffuse $\mathrm{X}$ - and gamma-ray radiation (in green) is the dominant background component below a few hundred $\mathrm{keV}$, but it is also a fundamental science topic for e-ASTROGAM above a few $\mathrm{MeV}$.

induced by fast particles (mainly leptons) impinging the spacecraft, as well as by the cosmic diffuse radiation and the atmospheric gamma-ray emission.

\section{Angular and spectral resolution}

e-ASTROGAM will image the Universe with substantially improved angular resolution both in the $\mathrm{MeV}$ domain and above a few hundreds of $\mathrm{MeV}$, i.e. improving the angular resolution of the $C G R O / C O M P T E L$ telescope and that of the Fermi/LAT instrument by a factor of $\sim 4$ at $1 \mathrm{MeV}$ and $1 \mathrm{GeV}$, respectively.

In the pair production domain, the PSF improvement over Fermi/LAT is due to (i) the absence of heavy converters in the Tracker, (ii) the light mechanical structure of this detector minimizing the amount of passive material within the detection volume and thus enabling a better tracking of the secondary electrons and positrons, and (iii) the analog readout of the DSSD signals allowing a fine spatial resolution of about $40 \mu \mathrm{m}(\sim 1 / 6$ of the microstrip pitch). In the Compton domain, thanks to the fine spatial and spectral resolutions of both the Tracker and the Calorimeter, the e-ASTROGAM angular resolution will be close to the physical limit induced by the Doppler broadening due to the velocity of the target atomic electrons.

Figure 2 shows an example of the e-ASTROGAM imaging capability in the $\mathrm{MeV}$ domain compared to COMPTEL. The e-ASTROGAM synthetic map of the Cygnus region was produced from the third Fermi LAT (3FGL) catalog of sources detected at photon energies $E_{\gamma}>100 \mathrm{MeV}$ [7, assuming a simple extrapolation of the measured power-law spectra to lower energies. 


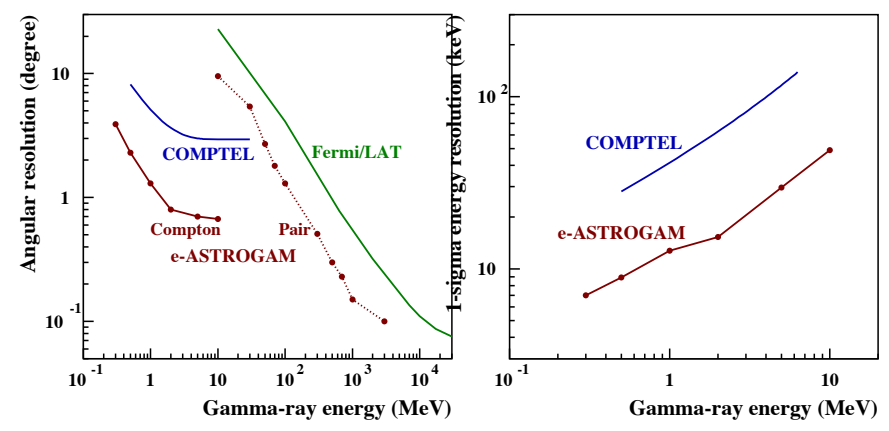

FIG. 19. Left panel - e-ASTROGAM on-axis angular resolution compared to that of COMPTEL and Fermi/LAT. In the Compton domain, the presented performance of eASTROGAM and COMPTEL is the FWHM of the angular resolution measure (ARM). In the pair domain, the point spread function (PSF) is the $68 \%$ containment radius for a $30^{\circ}$ point source. The Fermi/LAT PSF is from the Pass 8 analysis (release 2 version 6 ) and corresponds to the FRONT and PSF event type. Right panel $-1 \sigma$ energy resolution of COMPTEL and e-ASTROGAM in the Compton domain after event reconstruction and selection on the ARM.

It is clear from this example that e-ASTROGAM will substantially overcome (or eliminate in some cases) the confusion issue that severely affected the previous and current generations of gamma-ray telescopes. The eASTROGAM imaging potential will be particularly relevant to study the various high-energy phenomena occurring in the GC region.

e-ASTROGAM will also significantly improve the energy resolution with respect to COMPTEL, e.g. by a factor of $\sim 3.2$ at $1 \mathrm{MeV}$, where it will reach a $1 \sigma$ resolution of $\Delta E / E=1.3 \%$ (Figure 19). In the pair production domain above $30 \mathrm{MeV}$, the simulated spectral resolution is within $20-30 \%$.

\section{Field of View}

The e-ASTROGAM field of view was evaluated from detailed simulations of the angular dependence of the sensitivity. Specifically, the width of the field of view was calculated as the half width at half maximum (HWHM) of the inverse of the sensitivity distribution as a function of the polar, off-axis angle, for a constant azimuthal angle $\phi=22.5^{\circ}$. In the Compton domain, the sensitivity remains high within $40^{\circ}$ to $50^{\circ}$ off-axis angle and then degrades for larger incident angles. For example, the field of view at $1 \mathrm{MeV}$ amounts to $46^{\circ} \mathrm{HWHM}$, with a fraction-of-sky coverage in zenith pointing mode of $23 \%$, corresponding to $\Omega=2.9$ sr.

In the pair-production domain, the field-of-view assessment is also based on in-flight data from the AGILE and Fermi-LAT gamma-ray imager detectors. With the eASTROGAM characteristics (size, Si plane spacing, overall geometry), the field of view is found to be $>2.5 \mathrm{sr}$ above $10 \mathrm{MeV}$.

\section{Effective area and continuum sensitivity}

Improving the sensitivity in the medium-energy gamma-ray domain $(1-100 \mathrm{MeV})$ by one to two orders of magnitude compared to previous missions is the main requirement for the proposed e-ASTROGAM mission. Such a performance will open an entirely new window for discoveries in the high-energy Universe. Tables III and IV present the simulated effective area and continuum sensitivity in the Compton and pair-production domains. The sensitivity below $10 \mathrm{MeV}$ is largely independent of the source location (inner galaxy vs. high latitude), because the diffuse gamma-ray background is not a major background component in the Compton domain.

Figure 1 shows the e-ASTROGAM continuum sensitivity for a 1-year effective exposure of a high Galactic latitude source. Such an effective exposure will be reached for broad regions of the sky after 3 years of operation, given the very large field of view of the instrument. We see that e-ASTROGAM would provide an important leap in sensitivity over a wide energy band, from about $200 \mathrm{keV}$ to $100 \mathrm{MeV}$. At higher energies, e-ASTROGAM would also provide a new vision of the gamma-ray sky thanks to its angular resolution, which would reduce the source confusion that plagues the current Fermi-LAT and AGILE images near the Galactic plane (see, e.g., the 3FGL catalog [7]).

\section{Line sensitivity}

Table $\mathrm{V}$ shows the e-ASTROGAM $3 \sigma$ sensitivity for the detection of key gamma-ray lines from pointing observations, together with the sensitivity of the INTEGRAL Spectrometer (SPI). The latter was obtained from the INTEGRAL Observation Time Estimator (OTE) assuming $5 \times 5$ dithering observations. The reported line widths are from SPI observations of the 511 and $847 \mathrm{keV}$ lines (SN 2014J), and from theoretical predictions for the other lines. Noteworthy, the neutron capture line from accreting neutron stars can be significantly redshifted and broadened (FWHM between 10 and $100 \mathrm{keV}$ ) depending on the geometry of the mass accretion [31.

We see that e-ASTROGAM will achieve a major gain in sensitivity compared to SPI for all gamma-ray lines, the most significant improvement being for the $847 \mathrm{keV}$ line from Type Ia SNe (see Sect. II). With the predicted line sensitivity, e-ASTROGAM will also (i) provide a much better map of the $511 \mathrm{keV}$ radiation from positron annihilation in the inner Galaxy, (ii) uncover $\sim 10$ young, ${ }^{44}$ Ti-rich SN remnants in the Galaxy and thus provide new insight on the explosion mechanism of core-collapse $\mathrm{SNe}$ (iii) detect for the first time the expected [46 line from ${ }^{22} \mathrm{Na}$ decay in novae hosted by $\mathrm{ONe}$ white dwarfs, (iv) provide a new constraint on the nuclear equation of state of neutron stars by detecting the predicted 31] redshifted $2.2 \mathrm{MeV}$ line from Scorpius X-1, and (iv) measure the energy density of low-energy cosmic rays in the inner 
TABLE III. e-ASTROGAM performance in the Compton domain simulated with MEGAlib v2.26.01. The 3 $\sigma$ continuum sensitivity is for the detection of a point source on axis after an observation time $T_{\text {obs }}=10^{6} \mathrm{~s}$.

\begin{tabular}{|c|c|c|c|c|c|c|}
\hline $\begin{array}{c}\mathbf{E} \\
(\mathrm{MeV})\end{array}$ & $\underset{(\mathrm{MeV})}{\Delta \mathrm{E} \text { spectrum }^{(\mathrm{a})}}$ & $\begin{array}{c}\text { Angular } \\
\text { selection }\end{array}$ & $\begin{array}{l}\text { Effective area } \\
\text { after selection } \\
\left(\mathrm{cm}^{2}\right)\end{array}$ & $\begin{array}{l}\text { Background rate } \\
\text { after selection } \\
\left(\text { count s } \mathbf{s}^{-1}\right)\end{array}$ & $\begin{array}{c}\text { Sensitivity } \\
\left(\text { photon } \mathrm{cm}^{-2} \mathrm{~s}^{-1}\right)\end{array}$ & Notes \\
\hline 0.3 & $0.15-0.45$ & $4.3^{\circ}$ & 560 & 28 & $2.8 \times 10^{-5}$ & $\begin{array}{c}\text { Without e- } \\
\text { tracking }\end{array}$ \\
\hline 0.5 & $0.25-0.75$ & $2.5^{\circ}$ & 446 & 3.5 & $1.3 \times 10^{-5}$ & $\begin{array}{c}\text { Without e- } \\
\text { tracking }\end{array}$ \\
\hline 1 & $0.5-1.5$ & $1.5^{\circ}$ & 297 & 1.4 & $1.2 \times 10^{-5}$ & $\begin{array}{c}\text { Without e- } \\
\text { tracking }\end{array}$ \\
\hline 2 & $1.0-3.0$ & $1.1^{\circ}$ & 117 & 0.097 & $8.0 \times 10^{-6}$ & $\begin{array}{l}\text { With e- } \\
\text { tracking }\end{array}$ \\
\hline 5 & $2.5-7.5$ & $0.8^{\circ}$ & 105 & 0.031 & $5.0 \times 10^{-6}$ & $\begin{array}{l}\text { With e- } \\
\text { tracking }\end{array}$ \\
\hline 10 & $5-15$ & $0.8^{\circ}$ & 50 & 0.007 & $5.0 \times 10^{-6}$ & $\begin{array}{l}\text { With e- } \\
\text { tracking }\end{array}$ \\
\hline
\end{tabular}

(a) Source spectrum is an $E^{-2}$ power-law in the range $\Delta \mathrm{E}$.

(b) ARM radius. Note that the best sensitivity results are obtained for a selection on the ARM radius slightly larger than the optimal ARM.

(c) Effective area after event selection optimized for sensitivity.

(d) Total background including the atmospheric $\gamma$-ray background, the cosmic $\gamma$-ray background, the activation induced by primary and semi-trapped particles (mainly protons), and the prompt reactions from primary (i.e. cosmic-ray) protons, as well as from secondary protons and leptons (electrons and positrons).

TABLE IV. e-ASTROGAM performance in the pair-production domain simulated with BoGEMMS v2.0.1, together with Kalman v1.5.0 and Trigger v1.0.0. All results are for a $30^{\circ}$ off-axis source and for $T_{\mathrm{obs}}=10^{6} \mathrm{~s}$. The King function used to fit the PSF, derived from the model of XMM data, is defined, e.g., in 83.

\begin{tabular}{|c|c|c|c|c|c|c|c|c|}
\hline $\begin{array}{c}\mathbf{E} \\
(\mathrm{MeV})\end{array}$ & $\begin{array}{c}\Delta \mathbf{E} \\
\text { spectrum }^{(\mathrm{a})} \\
(\mathrm{MeV})\end{array}$ & $\mathbf{P S F}^{(b)}$ & $\begin{array}{c}\text { Effective } \\
\underset{\operatorname{area}}{(c)} \\
\left(\mathrm{cm}^{2}\right)\end{array}$ & $\begin{array}{c}\text { Inner } \\
\text { Galaxy } \\
\text { Backgr. rate } \\
\left(\text { count s }^{-1}\right) \\
\end{array}$ & $\begin{array}{c}\text { Inner } \\
\text { Galaxy } \\
\text { Sensitivity } \\
\left(\text { ph } \mathrm{cm}^{-2} \mathbf{s}^{-1}\right) \\
\end{array}$ & 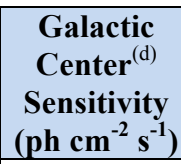 & \begin{tabular}{|c|} 
Extragal. \\
Backgr. \\
rate \\
$\left(\right.$ count s $\left.^{-1}\right)$ \\
\end{tabular} & 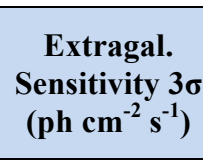 \\
\hline 10 & $7.5-15$ & $9.5^{\circ}$ & 215 & $3.4 \times 10^{-2}$ & $7.7 \times 10^{-6}$ & $1.3 \times 10^{-5}$ & $3.8 \times 10^{-3}$ & $2.6 \times 10^{-6}$ \\
\hline 30 & $15-40$ & $5.4^{\circ}$ & 846 & $1.6 \times 10^{-2}$ & $1.4 \times 10^{-6}$ & $2.4 \times 10^{-6}$ & $1.6 \times 10^{-3}$ & $4.3 \times 10^{-7}$ \\
\hline 50 & $40-60$ & $2.7^{\circ}$ & 1220 & $4.0 \times 10^{-3}$ & $4.6 \times 10^{-7}$ & $8.0 \times 10^{-7}$ & $3.4 \times 10^{-4}$ & $1.4 \times 10^{-7}$ \\
\hline 70 & $60-80$ & $1.8^{\circ}$ & 1245 & $1.3 \times 10^{-3}$ & $2.6 \times 10^{-7}$ & $4.5 \times 10^{-7}$ & $1.0 \times 10^{-4}$ & $7.2 \times 10^{-8}$ \\
\hline 100 & $80-150$ & $1.3^{\circ}$ & 1310 & $5.1 \times 10^{-4}$ & $1.6 \times 10^{-7}$ & $2.7 \times 10^{-7}$ & $3.2 \times 10^{-5}$ & $3.9 \times 10^{-8}$ \\
\hline 300 & $150-400$ & $0.51^{\circ}$ & 1379 & $4.8 \times 10^{-5}$ & $4.5 \times 10^{-8}$ & $7.8 \times 10^{-8}$ & $1.1 \times 10^{-6}$ & $6.9 \times 10^{-9}$ \\
\hline 500 & $400-600$ & $0.30^{\circ}$ & 1493 & $1.4 \times 10^{-5}$ & $2.2 \times 10^{-8}$ & $3.8 \times 10^{-8}$ & $1.8 \times 10^{-7}$ & $3.3 \times 10^{-9}$ \\
\hline 700 & $600-800$ & $0.23^{\circ}$ & 1552 & $6.3 \times 10^{-6}$ & $1.5 \times 10^{-8}$ & $2.5 \times 10^{-8}$ & $7.6 \times 10^{-8}$ & $3.2 \times 10^{-9}$ \\
\hline 1000 & $800-2000$ & $0.15^{\circ}$ & 1590 & $2.1 \times 10^{-6}$ & $8.3 \times 10^{-9}$ & $1.4 \times 10^{-8}$ & $2.1 \times 10^{-8}$ & $3.1 \times 10^{-9}$ \\
\hline 3000 & $2000-4000$ & $0.10^{\circ}$ & 1810 & $3.3 \times 10^{-7}$ & $2.9 \times 10^{-9}$ & $5.0 \times 10^{-9}$ & $2.9 \times 10^{-9}$ & $2.8 \times 10^{-9}$ \\
\hline
\end{tabular}

(a) Source spectrum is an $E^{-2}$ power-law in the range $\Delta \mathrm{E}$.

(b) Point Spread Function (68\% containment radius) derived from a single King function fit of the angular distribution.

(c) Effective area after event selection.

(d) The background for the Galactic Center is assumed to be 3 times larger than that of the Inner Galaxy.

Galaxy to better understand the role of these particles in the Galactic ecosystem.
6. Polarization response

Both Compton scattering and pair creation partially preserve the linear polarization information of incident photons. In a Compton telescope, the polarization sig- 
TABLE V. e-ASTROGAM line sensitivity $\left(3 \sigma\right.$ in $\left.10^{6} \mathrm{~s}\right)$ compared to that of INTEGRAL/SPI[118].

\begin{tabular}{|c|c|c|c|c|c|}
\hline $\begin{array}{c}\mathrm{E} \\
(\mathbf{k e V})\end{array}$ & $\begin{array}{c}\text { FWHM } \\
(\mathrm{keV})\end{array}$ & Origin & $\begin{array}{l}\text { SPI sensitivity } \\
\left(\mathrm{ph} \mathrm{cm}^{-2} \mathrm{~s}^{-1}\right)\end{array}$ & $\begin{array}{c}\text { e-ASTROGAM } \\
\text { sensitivity } \\
\left(\mathrm{ph} \mathrm{cm}^{-2} \mathrm{~s}^{-1}\right) \\
\end{array}$ & $\begin{array}{c}\text { Improvement } \\
\text { factor }\end{array}$ \\
\hline 511 & 1.3 & $\begin{array}{l}\text { Narrow line component of the } \\
\mathrm{e}+/ \mathrm{e}-\text { annihilation radiation from } \\
\text { the Galactic center region }\end{array}$ & $5.2 \times 10^{-5}$ & $4.1 \times 10^{-6}$ & 13 \\
\hline 847 & 35 & ${ }^{56} \mathrm{Co}$ line from thermonuclear $\mathrm{SN}$ & $2.3 \times 10^{-4}$ & $3.5 \times 10^{-6}$ & 66 \\
\hline 1157 & 15 & $\begin{array}{l}{ }^{44} \mathrm{Ti} \text { line from core-collapse SN } \\
\text { remnants }\end{array}$ & $9.6 \times 10^{-5}$ & $3.6 \times 10^{-6}$ & 27 \\
\hline 1275 & 20 & $\begin{array}{l}{ }^{22} \mathrm{Na} \text { line from classical novae of } \\
\text { the ONe type }\end{array}$ & $1.1 \times 10^{-4}$ & $3.8 \times 10^{-6}$ & 29 \\
\hline 2223 & 20 & $\begin{array}{l}\text { Neutron capture line from } \\
\text { accreting neutron stars }\end{array}$ & $1.1 \times 10^{-4}$ & $2.1 \times 10^{-6}$ & 52 \\
\hline 4438 & 100 & $\begin{array}{l}{ }^{12} \mathrm{C} \text { line produced by low-energy } \\
\text { Galactic cosmic-ray in the } \\
\text { interstellar medium }\end{array}$ & $1.1 \times 10^{-4}$ & $1.7 \times 10^{-6}$ & 65 \\
\hline
\end{tabular}
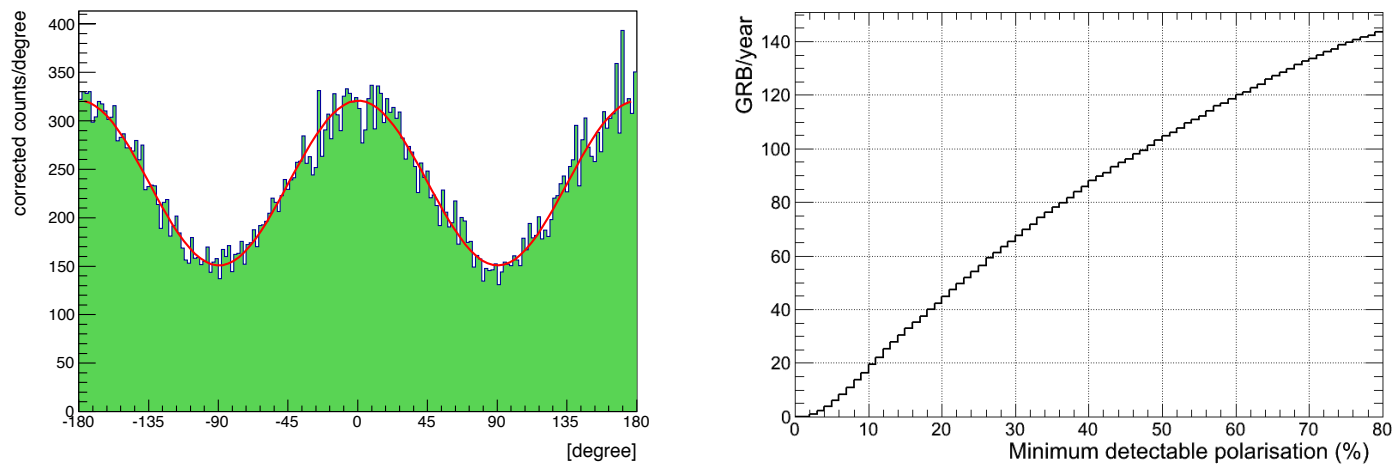

FIG. 20. Left panel-e-ASTROGAM polarization response (polarigramme) in the $0.2-2 \mathrm{MeV}$ range for a $100 \%$ polarized, 10 mCrab-like source observed on axis for $10^{6} \mathrm{~s}$. The corresponding modulation is $\mu_{100}=0.36$. Right panel-Cumulative number of GRBs to be detected by e-ASTROGAM as a function of the minimum detectable polarization at the $99 \%$ confidence level.

nature is reflected in the probability distribution of the azimuthal scattering angle. In the pair domain, the polarization information is given by the distribution of azimuthal orientation of the electron-positron plane (as discussed since the 1950s; see, e.g., [29, [147, 150]). eASTROGAM will be able to perform for the first time at these energies polarization measurements thanks to the fine 3D position resolution of both the Si Tracker and the Calorimeter, as well as the light mechanical structure of the Tracker, which is devoid of any heavy absorber in the detection volume.

The left panel of Figure 20 shows an example of a polarigramme in the $0.2-2 \mathrm{MeV}$ range (i.e. in the Compton domain), simulated with MEGAlib. The calculations assume a $100 \%$ polarized emission from a 10 mCrab-like source observed on axis. The systematic effects of instrumental origin were corrected by simulating the azimuthal response of the instrument to an unpolarized source with the same spectral distribution and position in the field of view as the polarized source. From the obtained modu- lation $\left(\mu_{100}=0.36\right)$, we find that at low energies $(0.2-$ $2 \mathrm{MeV}$ ), e-ASTROGAM will be able to achieve a Minimum Detectable Polarization (MDP) at the $99 \%$ confidence level as low as $0.7 \%$ for a Crab-like source in $1 \mathrm{Ms}$ (statistical uncertainties only). After one year of effective exposure of the GC region, the achievable $\mathrm{MDP}_{99}$ for a $10 \mathrm{mCrab}$ source will be $10 \%$. With such a performance, e-ASTROGAM will be able to study the polarimetric properties of many pulsars, magnetars, and black hole systems in the Galaxy.

The right panel of Figure 20 shows the number of GRBs detectable by e-ASTROGAM as a function of $\mathrm{MDP}_{99}$ in the $150-300 \mathrm{keV}$ band. The total number of GRBs detected by e-ASTROGAM will be $\sim 600$ in 3 years of nominal mission lifetime. Here, the GRB emission spectrum has been approximated by a typical Band function 25] with $\alpha=-1.1, \beta=-2.3$, and $E_{\text {peak }}=0.3 \mathrm{MeV}$, and the response of e-ASTROGAM to linearly polarized GRBs has been simulated at several off-axis angles in the range $\left[0^{\circ} ; 90^{\circ}\right]$. The number of GRBs with polar- 
ization measurable with e-ASTROGAM has then been estimated using the Fourth BATSE GRB Catalog 105. We see in Figure 20 that e-ASTROGAM should be able to detect a polarization fraction of $20 \%$ in about $42 \mathrm{GRBs}$ per year, and a polarization fraction of $10 \%$ in $~ 16$ GRBs per year. This polarization information, combined with spectroscopy over a wide energy band, will provide unambiguous answers to fundamental questions on the sources of the GRB highly relativistic jets and the mechanisms of energy dissipation and high-energy photon emission in these extreme astrophysical phenomena.

The measurement of polarization using the azimuthal orientation of the electron-positron plane is complex and a precise evaluation of the unfolding procedures and performance requires accurate simulation and testing [30. 138. Some estimates are however possible. The expected performance for GRBs has been already discussed in II A 1. From the formula derived in 39] and taking into account the angular opening of pairs [82], the multiple scattering of the secondary electron and positron [102, one can estimate, using a simplified model for the conversions of photons in the energy range from $20 \mathrm{MeV}$ to $200 \mathrm{MeV}$, that $\sim 20 \%$ polarization from Vela could be detected at $3 \sigma$ in 15 months of data. Also using a simplified model for pair production and multiple scattering of electrons and positrons, multiple scattering simplified model), a MDP of $\sim 45 \%$ at $3 \sigma$ has been estimated for the Crab Nebula in $10^{6} \mathrm{~s}$ in the range from 10 to $100 \mathrm{MeV}$. These should be considered as lower limit MDP figures.

\section{Technology readiness}

e-ASTROGAM is based on the heritage of AGILE and of the Fermi-LAT, with an overall simpler structure (the division into 4 tower units instead of the 16 of LAT greatly reduces complexity); it uses technology developed for previous space-based detectors. Most components are at or above TRL 6 .

The DSSDs have proven their performance in PAMELA 17] and AMS-02 22. Low-power ASICs suitable for the readout of the were already qualified and used on ASTRO-H/HXI [100]. The low-power SiPM sensors selected for the ACD and the solid-state detectors foreseen for the CsI calorimeter have not yet flown on satellite missions but are in use in suborbital prototypes 33] and are planned for use on future space-borne platforms.

\section{MISSION CONFIGURATION AND PROFILE}

\section{A. Orbit and launcher}

The best orbit for e-ASTROGAM is an equatorial LEO (required to have an inclination $i<2.5^{\circ}$, and eccentricity $e<0.01$ ) of altitude $550-600 \mathrm{~km}$ : as already discussed, particle background properties are optimum for this orbit. Such an orbit allows making use of the ESA ground station at Kourou as well as of the ASI Malindi station in Kenya.

The foreseen launcher for e-ASTROGAM is Ariane 6.2. The launcher fairing (5.4 $\mathrm{m}$ diameter) and performance for the targeted orbit ( $>5$ tons) are well adapted to the spacecraft dimensions $\left(\mathrm{L} \times \mathrm{W} \times \mathrm{H}=340 \times 218 \times 371 \mathrm{~cm}^{3}\right.$; Figure 21) and separated mass $(2680 \mathrm{~kg}$, including maturity margins at sub-system level and an additional system margin of $20 \%$ )

\section{B. Spacecraft and system requirements}

The e-ASTROGAM system is composed of a satellite and a ground segment that includes the ESA ground station at Kourou and possibly the ASI Malindi station in Kenya. These stations are in charge of performing the spacecraft control, monitoring, and the acquisition of scientific data. Communication with the ground is ensured by an X-band telecommand and telemetry subsystem. The average orbital contact time with the two ground stations is about $10 \mathrm{~min}$ for each of them. The average data generation of both payload $(\mathrm{P} / \mathrm{L})$ and platform amounts to $1382 \mathrm{kbps}$, taking into account a compression of the raw $\mathrm{P} / \mathrm{L}$ data by a factor of 2.6 , as obtained with e-ASTROGAM representative science data and standard compression programs. The overall data generation rate is then about 8.0 Gbit per orbit, which can be transmitted using the two ground stations at a downlink rate of $\sim 6.6 \mathrm{Mbps}$, in agreement with the bandwidth limit in X-band for e-ASTROGAM mission category (maximum downlink rate of $8.5 \mathrm{Mbps}$ ).

The e-ASTROGAM spacecraft is observing the sky according to a predefined pointing plan uploaded from ground. Different pointing profiles can be selected in order to observe selected sky regions or to perform a scanning that can cover a large fraction of the sky at each orbit.

The spacecraft platform is made of a structure that mechanically supports the e-ASTROGAM instrument and hosts internally the payload electronic units and all the platform subsystems. The payload is attached to a mechanical structure at a distance of about $94 \mathrm{~cm}$ from the top of the platform. The space between the payload and the platform is used to (i) host the time-of-flight (ToF) unit of the $\mathrm{P} / \mathrm{L}$ Anticoincidence (AC) system, (ii) host the P/L PSU, PDHU and BEE modules (Tracker, Calorimeter and $\mathrm{AC} \mathrm{BEEs}$ ), and (iii) accommodate the two fixed radiators of the thermal control system. In addition, this mechanical design has the advantage of significantly reducing the instrument background due to prompt and delayed gamma-ray emissions from fast particle reactions with the platform materials.

Deployable and steerable solar panels are required to support the payload operating profile and the platform pointing and communication requirements. Figure 22 shows the spacecraft configuration in flight with deployed solar arrays. Figure 23 shows e-ASTROGAM under Ari- 


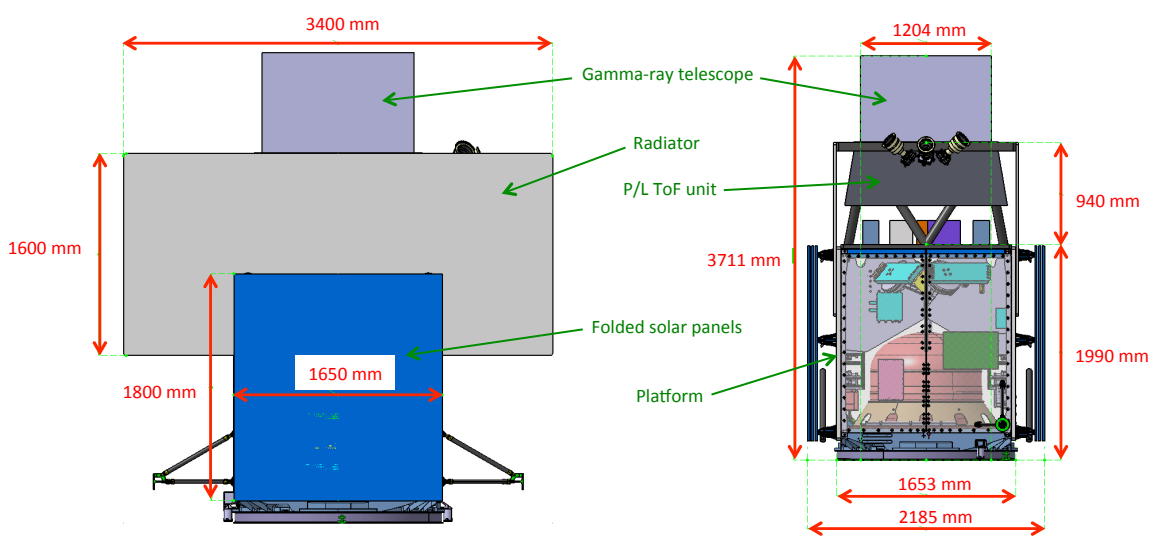

FIG. 21. e-ASTROGAM dimensions. The space between the payload (P/L) ToF unit and the spacecraft hosts the P/L PSU, PDHU and BEE modules.

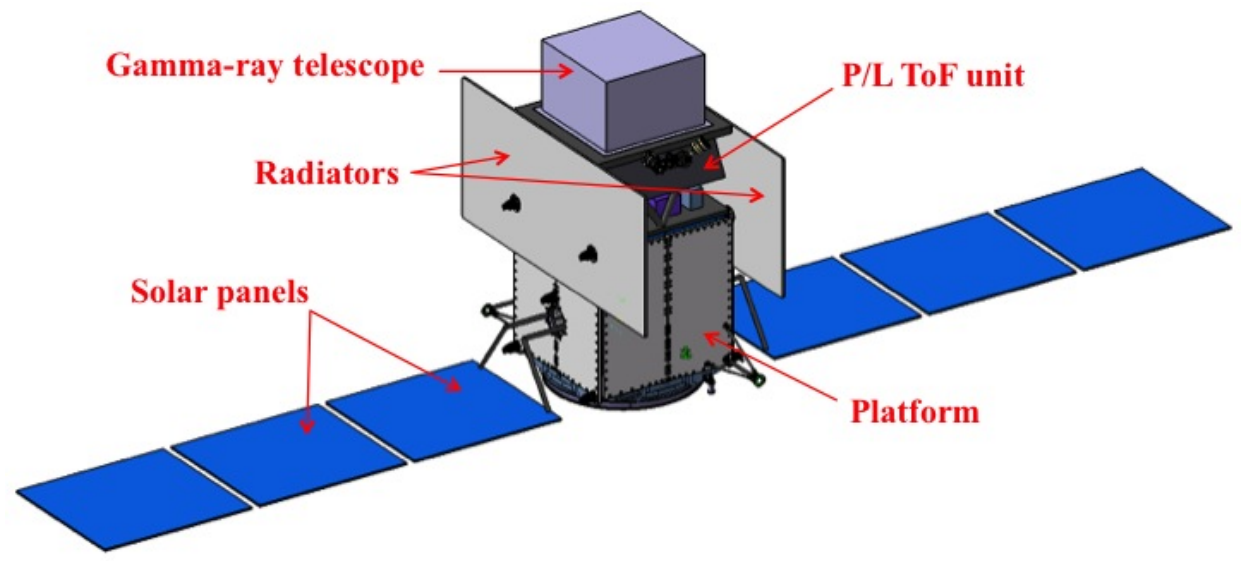

FIG. 22. e-ASTROGAM spacecraft in deployed configuration.

ane 6.2 fairing in upper position of a dual launch configuration.

A precise timing of the payload data $(1 \mu \mathrm{s}$ at $3 \sigma)$ is required to perform a proper on ground data processing able to guarantee the scientific performance of the mission. The required timing performance is obtained by a GPS delivering a pulse-per-second (PPS) signal both to the $\mathrm{P} / \mathrm{L} \mathrm{PDHU}$ and the Spacecraft Management Unit time management in order to allow a fine synchronization with the time reference.

\section{Attitude and Orbital Control Systems}

The Attitude and Orbital Control Systems (AOCS) performance requirements for the e-ASTROGAM mission are not critical, with an absolute Pointing Error of less than 1 degree, a relative Pointing Error of less than $0.01 \mathrm{deg} / \mathrm{s}$ and an Absolute knowledge Error of less than 30 arcseconds. In addition, to fulfill the scientific requirements, the spacecraft shall be placed in a LEO without the need to perform orbit maintenance during nominal lifetime. The propulsion subsystem is therefore provided

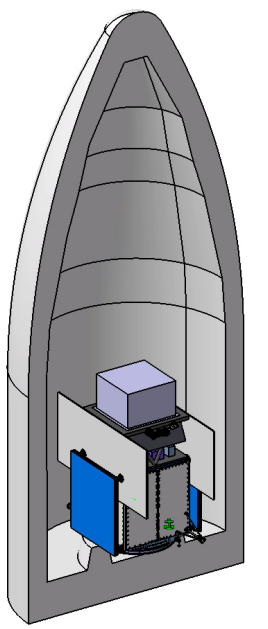

FIG. 23. e-ASTROGAM under Ariane 6.2 fairing in upper position.

with the only purpose to have the possibility to correct for launcher dispersion, perform debris avoidance and, as 
required by the international regulations and because of the construction of the instrument, to execute a direct controlled re-entry at the end of the mission. The proposed subsystem design is a monopropellant hydrazine, blow-down mode propulsion system. The hydrazine is contained in a diaphragm tank, together with the pressurant. From a preliminary estimation of the propellant budget the amount of hydrazine required to fulfill the mission needs are about $266 \mathrm{~kg}$, among which more than $190 \mathrm{~kg}$ are allocated to the end of mission disposal.

The spacecraft is able to provide the following attitude pointings to support the payload observation requirements:

- zenith pointing to perform at each orbit a scan of the sky;

- nearly inertial pointing (with the possibility to slowly rotate around the payload boresight) to observe continuously a selected area of the sky;

- fast payload repointing during eclipse periods to avoid the presence of the Earth in the payload FoV (allowing 2 pointings per orbit).

\section{Thermal control system}

The required pointing accuracy $\left( \pm 1^{\circ}\right)$, stability $\left(0.01^{\circ} / \mathrm{s}\right)$, and attitude knowledge of $30 \operatorname{arcsec}$ (to be reached after ground processing) can be obtained using standard class sensors and actuators. The 3-axis stabilized attitude control is achieved mainly using a set of four reaction wheels used in zero momentum mode ensuring the possibility to perform fast repointing manoeuvres. Magnetic torquers are provided to perform wheels desaturation and to support a safe attitude pointing based on a basic subset of AOCS items.

The e-ASTROGAM P/L detector has an optimal performance in the temperature range $-10^{\circ}-0^{\circ}$. In order to guarantee the required environment, the $\mathrm{P} / \mathrm{L}$ power dissipation is evacuated towards external space by two fixed large radiators located on the two solar array panels, below the instrument. The total radiative area is $11.6 \mathrm{~m}^{2}$. The radiator is a heat transfer device based on a Loop Heat Pipe (LHP) with a condenser as a part of a radiation heat exchanger. Large radiators are necessary because of the large payload thermal dissipation, variable external environmental conditions, and limited heat transport capability within the payload. However, thanks to the large Ariane 6.2 fairing ( $4.5 \mathrm{~m}$ diameter), a deployable radiator can be avoided, as shown in Figure 23 .

\section{SUMMARY}

e-ASTROGAM is a concept for a gamma-ray space observatory that can revolutionize the astronomy of medium/high-energy gamma rays by increasing the number of known sources in this field by more than an order of magnitude and providing polarization information for many of these sources - thousands of sources are expected to be detected during the first 3 years of operations. Furthermore, the proposed wide-field gamma-ray observatory will play a major role in the development of timedomain astronomy, and provide valuable information for the localization and identification of gravitational wave sources.

The instrument is based on an innovative design, which minimizes any passive material in the detector volume. The instrument performance has been assessed through detailed simulations using state-of-the-art tools and the results fully meet the scientific requirements of the proposed mission.

e-ASTROGAM will operate as an observatory open to the international community. The gamma-ray observatory will be complementary to ground and space instruments, and multifrequency observation programs will be very important for the success of the mission. In particular, e-ASTROGAM will be essential for investigations jointly done with radio (VLA, VLBI, ALMA, SKA), optical (JWST, E-ELT and other ground telescopes), X-ray and $\mathrm{TeV}$ ground instrument (CTA, HAWC, LHAASO and other ground-based detectors). Special emphasis will be given to fast reaction to transients and rapid communication of alerts. New astronomy windows of opportunity (sources of gravitational waves, neutrinos, ultra high-energy cosmic rays) will be fully and uniquely explored.

\section{ACKNOWLEDGMENTS}

The contribution by P. Couzin (TAS-F), G. Cluzet (TAS-F), X. Roser (TAS-F), A. Laurens (CNES), D. Delrieu (CNES), M.-F. DelCastillo (CNES), C. Contini (CGS), P. Lattanzi (CGS), B. Morelli (CGS), A. Spalla (CGS), is acknowledged.

Comments from E. Orlando were appreciated.

The research leading to these results has received funding from the European Union's Horizon 2020 Programme under the AHEAD project (grant agreement n. 654215).
[1] Aartsen, M.G., et al., 2013, Science 342, 1242856

[2] Abbott, B.P., et al., 2016, Phys. Rev. Lett., 116, 061102
[3] Abdo A.A., et al., 2009, Science, 326, 1512.

[4] Abdo, A.A., et al., 2010, Astrophys. J., 709, 152 
[5] Abdo A.A., et al., 2011, Science, 331, 739

[6] Acero, F., et al., 2016, Astrophys. J., 224, 8

[7] Acero, F., et al., 2015, ApJS, 218, 23

[8] Ackermann, M., et al., 2011, Science, 334, 1103

[9] Ackermann, M., et al., 2013, Science, 339, 807

[10] Ackermann, M., et al., 2014, Phys. Rev. D, 89, 042001

[11] Ackermann, M., et al., 2014, Science, 345, 554

[12] Ackermann, M., et al., 2014, Astrophys. J., 793, 64

[13] Ackermann, M., et al., 2015, Astrophys. J., 799, 1

[14] Ackermann, M. et al. 2015, Phys. Rev. Lett,, 115, 231301

[15] Ackermann, M., et al., 2016, Astrophys. J., 824, 2

[16] Ackermann, M., et al., 2016, A\&A, 586, A71

[17] Adriani, O., et al., 2003, Nucl. Instr. Methods A, 511, 72

[18] Ahangarianabhari, M. et al., 2015, Nucl. Instr. Methods A, 770,155

[19] Ajello, M., et al., 2009, Astrophys. J., 699, 603

[20] Ajello, M., et al., 2012, Astrophys. J., 751, 108.

[21] Albert, A. et al., 2014, J. Cosmology Astropart. Phys., 1410,023

[22] Alcaraz, J., Alpat, B., Ambrosi, G., et al., 2008, Nucl. Instr. Methods A, 593, 376

[23] Arik, E., et al., 2009, J. Cosmology Astropart. Phys., 02, 008

[24] Bagliesi, M. G. et al., 2011, Nuclear Physics B Proceedings Supplements, 215, 344

[25] Band, D., et al., 1993, Astrophys. J., 413, 281

[26] Baumgartner, W.H., et al., 2013 ApJS, 207, 19

[27] Benhabiles-Mezhoud, H., et al., 2013, Astrophys. J., 763, 98

[28] Bergström, L., 1988, Nucl. Phys., B325, 647

[29] Berlin, T. H. \& Madansky, L., 1950, Phys. Rev., 78, 623

[30] Bernard, D., 2013, Nuclear Instr. and Methods in Phys. Res. A, 729, 765

[31] Bildsten, L., Salpeter, E. E., \& Wasserman, I., 1993, Astrophys. J., 408, 615

[32] Bird, A.J., et al., 2010, ApJS, 186, 1

[33] Bloser, P.F., et al., 2016, Nucl. Instr. Methods A, 812, 92

[34] Boddy, K. K. \& Kumar, J. 2016, AIP Conf. Proc. 1743, 020009

[35] Boehm, C.T., Ensslin, A. \& Silk, J., 2004, J. Phys. G, 30,279

[36] Boehm, C.T., et al., 2004, Phys. Rev. Lett., 92, 101301

[37] Breitschwerdt, D., et al., 1991, A\&A, 245, 79B

[38] Bringmann, T., et al., 2016, arXiv:1610.04613

[39] Buehler, R., et al.., 2010, "Measuring polarization of gamma-rays with Fermi", Presented at SciNeGHE Trieste, http://scineghe2010.ts.infn.it/programmaScientifico.php

[40] Bulgarelli, A., et al., 2012, Proceedings of the SPIE 8453, 845335

[41] Campana, R., et al., 2014, Experimental Astronomy, 37, 599

[42] Carlson, E. \& Profumo, S., 2014, Phys. Rev. D, 90, 023015

[43] Cheung, C.C., et al., 2016, Astrophys. J., 826, 142

[44] Churazov, E., et al., 2014, Nature, 512, 406

[45] Churazov, E., et al., 2015, Astrophys. J., 812, 62

[46] Clayton, D.D. \& Hoyle, F., 1974, Astrophys. J., 187, L101

[47] Crocker, R. \& Aharonian, F., 2011, Phys. Rev. Lett., 106,1102
[48] De Angelis, A., Mansutti, O. \& Roncadelli, M., 2008, Phys. Lett. B, 659, 847

[49] De Angelis, A. \& Pimenta, M.J., 2015, "Introduction to Particle and Astroparticle Physics - Questions to the Universe" (Springer)

[50] De Angelis A., Tatischeff, V. \& Giusti, M. (eds.), 2017, "e-ASTROGAM scientific workshop", eBook (Lulu), http://www.lulu.com/shop/alessandro-de-angelis/eastrogam-scientific-workshop/ebook/product23158421.html

[51] Danzmann, K., et al., eLISA White Paper, https://www.elisascience.org/multimedia/ document/white-paper-pdf

[52] Diehl, R., 2013, Rep. Progr. Phys., 76, 2, id. 026301

[53] Diehl, R., et al., 2014, Science, 345, 1162

[54] Diehl, R., et al., 2015, A\&A, 574, A72

[55] Diehl, R. \& Timmes, F.X., 1998, PASP, 110, 748

[56] Essig, R. et al., 2013, JHEP, 1, 193

[57] Everett, J., et al., 2008, Astrophys. J., 674, 258

[58] Forot, M., et al., 2008, Astrophys. J., 688, L29

[59] Fox, A., et al., 2015, Astrophys. J., 799, 7

[60] Funk, S., 2016, Ann. Rev. Nucl. Part. Sci. 65, 245

[61] Gal-Yam, A., et al., 2009, Nature, 462, 624

[62] Galanti, G. \& Roncadelli, M., 2013, arXiv:1305.2114

[63] Gatti, E. \& Rehak, P., 1984, Nucl. Instr. Methods A, 225, 608; www.pnsensor.de/Welcome/Detectors/SDD/

[64] Gevin, O. et al., 2012, Nuclear Instruments and Methods in Physics Research A, 695, 415

[65] Ghisellini, G., et al., 2010, MNRAS, 405, 387

[66] Ghisellini, G., et al., 2013, MNRAS, 432, 2818

[67] Gómez, S. et al., 2016, Proceedings of the SPIE, 9899, 98990G, doi: 10.1117/12.2231095

[68] Gomez-Gomar, J., Hernanz, M., Jose, J., Isern, J., 1998, MNRAS, 296, 913

[69] Götz, D., Laurent, P., Antier, S., et al. 2014, MNRAS, 444,2776

[70] Grefenstette, B.W., et al., 2014, Nature, 506, 339

[71] Grenier, I.A., Black, J.H. \& Strong, A.W. 2015, ARA\&A, 53, 199

[72] Hernanz, M., Jose, J., 2004, New Astron. Rev., 48, 35

[73] Hillebrandt, W., Kromer, M., Röpke, F. \& Ruiter, A., 2013, Front. Phys. 8, 116

[74] Hillebrandt, W. \& Niemeyer, J.C., 2000, ARA\&A, 38, 191

[75] Indriolo, N. \& McCall, B., 2012, Astrophys. J., 745, 91

[76] Isern, J., et al., 2016, A\&A, 588, A67

[77] Jaeckel, J. \& Ringwald, A., 2010, Ann. Rev. Nucl. Part. Sci., 60, 405

[78] Jogler, T. \& Funk, S., 2016, Astrophys. J., 816, 100

[79] José, J. \& Hernanz, M., 2007, Journal of Physics G Nuclear Physics, 34, R431

[80] Kadler, M., et al., 2016, Nature Physics 12, 807

[81] Kanbach, G., et al., 2005, Nucl. Instr. Methods A, 541, 310

[82] Kerzendorf, W. \& Sim, S., 2014, MNRAS, 440, 387

[83] Kirsch, M.G.F., et al., 2004, "XMM-Newton (cross)calibration", arXiv:astro-ph/0407257

[84] Kissmann, R., et al., 2015, Astroparticle Physics, 70, 39

[85] Koljonen, K., et al., 2010, MNRAS, 406, 307

[86] Krause, M. G. H., et al., 2015, A\&A, 578, A113

[87] Kretschmer, K., et al., 2013, A\&A, 559, A99

[88] Labanti, C. et al., 2008, Proceedings of the SPIE, 7021, 702116

[89] Limongi, M. \& Chieffi, A., 2006, Astrophys. J., 647, 483 
[90] Limousin, O. et al.. 2005, IEEE Transactions on Nuclear Science, 52, 1595

[91] Marisaldi, M. et al., 2005, IEEE Transactions on Nuclear Science, 52, 1842

[92] McClelland, D., et al., 2015, LIGO Scientific Collaboration, Instrument Science White Paper, LIGO Document T1500290-v2

[93] McConnell, M. L. 2016, accepted for publication in New Astronomy Review, arXiv:1611.06579

[94] Meyer, M., et al., 2017, Phys. Rev. Lett. 118, 011103

[95] Moiseev, A.A., et al., 2007, Astroparticle Physics, 27, 339

[96] Moiseev, A.A., et al., 2007, arXiv:1508.07349

[97] Moskalenko, I.V., Porter, T.A., and Digel, S.W., 2006, Astrophys. J. 652, L65

[98] Nakar, E., 2007, Phys. Rep., 442, 166

[99] Nomoto, K., Thielemann, F.-K. \& Yokoi, K., 1984, Astrophys. J., 286, 644

[100] Odaka, H., et al., 2012, Nucl. Instr. Methods A, 695, 179

[101] Olive, K.A., et al., 2014, Chin. Phys. C, 38, 090001 and 2015 update

[102] Olsen, H., 1963, Phys. Rev., 131, 406

[103] Orlando, E., \& Strong, A., 2007, Astrophys. Space Sci. 309,359

[104] Orlando, E., \& Strong, A., 2008, Astron. Astrophys. 480, 847

[105] Paciesas, W. S., et al., 1999, ApJS, 122, 465

[106] Paliya, V. S. et al., 2016, Astrophys. J.825, 74

[107] Patricelli, B., et al., 2016, arXiv:1606.06124

[108] Perotti, F., et al., 2006, Nucl. Instr. Meth. Phys. Res. A, 556, 228

[109] Petrović, J., Pasquale, S.D., Zaharijaš, G., 2014, J. Cosmology Astropart. Phys., 10, 052

[110] Phillips, M. M., 1993, Astrophys. J., 413, L105

[111] Piano, G., et al., 2012, A\&A, 545, A110

[112] Prada, F., et al., 2004, Phys. Rev. Lett., 93, 241301

[113] Punturo, M., et al., 2010, Classical and Quantum Gravity, 27, 194002

[114] Recchia, S., et al., 2016, MNRAS, 462, L88

[115] Recchia, S., Blasi, P. \& Morlino, G., 2016, MNRAS, 462,4227

[116] Ringwald, A., Rosenberg, L.J. \& Rybka, G., 2016, "Axions and other similar particles", in Patrignani, C., et al. (Particle Data Group), Chin. Phys. C, 40, 100001

[117] Romero, G.E., Vieyro, F.L. \& Chaty, S., 2014, A\&A, 562, L7
[118] Roques, J.P., et al., 2003, A\&A, 411, L91

[119] Roques, J.P., et al., 2015, ApJL, 813, 22

[120] Rudaz, S., et al., 1986, Phys. Rev. Lett., 56, 2128

[121] Ruiz-Lapuente, P., et al., 2016, Astrophys. J., 820, 142

[122] Schlickeiser, R., et al., 2014, Astrophys. J., 787, 35

[123] Schönfelder, V., et al., 1996, A\&A, 120

[124] Senno, N., et al., 2016, Phys. Rev. D, 93, 083003

[125] Siegert, T., et al., 2016, Nature, 531, 341

[126] Siegert, T., et al., 2016, A\&A, 595, 25

[127] Skilling, J., \& Strong, A. W., 1976, A\&A, 53, 253 Nature, 454, 1096

[128] Tagliaferri, G., et al., 2015, Astrophys. J., 807, 167

[129] Takahashi, T., Uchiyama, Y., \& Stawarz, Ł. 2013, Astroparticle Physics, 43, 142

[130] Takami, H., Kyutoku, K., \& Ioka, K., 2014, Phys. Rev. $\mathrm{D}, 89,063006$

[131] Tanaka, T., et al., 2008 Astrophys. J., 685, 988-1004

[132] Tatischeff, V. \& Hernanz, M., 2007, Astrophys. J., 663, L101

[133] Tavani, M., et al., 2009, Nature, 462, 620

[134] Tavani, M., et al., 2009, A\&A, 502, 995

[135] Tavani, M., et al., 2011, Science, 331, 736

[136] Tavani, M., et al., 2013, Nucl. Phys. (Proc. Suppl.), 243-244, 131.

[137] The, L.-S. \& Burrows, A. 2014, Astrophys. J., 786, 141

[138] Tsai, Y.S., 1974, Rev. Mod. Phys., 46, 815

[139] Tsygankov, S. S., Krivonos, R. A., Lutovinov, A. A., et al. 2016, MNRAS, 458, 3411

[140] Uchiyama, Y., et al., 2012, Astrophys. J., 749, 35

[141] Veres, P. \& Meszaros, P. 2014, Astrophys. J., 787, 168

[142] Volonteri, M., et al., 2011, MNRAS, 416, 216.

[143] von Ballmoos, P., 2014, Hyperfine Interact., 228, 1-3, 91.

[144] Walker, M.G., et al., 2009, Astrophys. J., 704, 1274

[145] Wang, L.J., et al., 2016, Astrophys. J., 823, 15

[146] Wang, X. \& Loeb, A., 2016, Nature Physics, 12, 1116

[147] Wick, G.C., 1951, Phys. Rev., 81, 467

[148] Woosley, S.E., et al., Astrophys. J.662, 487

[149] Wouters, D. \& Brun, P., 2012, Phys. Rev. D, 86, 043005

[150] Yang, C.N., 1950, Phys. Rev., 77, 722

[151] Zdziarski, A.A., Stawarz, Ł., Pjanka, P. \& Sikora, M. 2014, MNRAS, 440, 2238

[152] Zhang, H. \& Boettcher, M., 2013, Astrophys. J., 774, 18

[153] Zoglauer, A., Andritschke, R. \& Schopper, F., 2006, New A Rev., 50, 629 